\title{
WestVirginiaUniversity
}

THE RESEARCH REPOSITORY @ WVU

Graduate Theses, Dissertations, and Problem Reports

2008

\section{Modeling, identification and control of a cold flow circulating fluidized bed}

Rupendranath Panday

West Virginia University

Follow this and additional works at: https://researchrepository.wvu.edu/etd

\section{Recommended Citation}

Panday, Rupendranath, "Modeling, identification and control of a cold flow circulating fluidized bed" (2008). Graduate Theses, Dissertations, and Problem Reports. 4413.

https://researchrepository.wvu.edu/etd/4413

This Thesis is protected by copyright and/or related rights. It has been brought to you by the The Research Repository @ WVU with permission from the rights-holder(s). You are free to use this Thesis in any way that is permitted by the copyright and related rights legislation that applies to your use. For other uses you must obtain permission from the rights-holder(s) directly, unless additional rights are indicated by a Creative Commons license in the record and/ or on the work itself. This Thesis has been accepted for inclusion in WVU Graduate Theses, Dissertations, and Problem Reports collection by an authorized administrator of The Research Repository @ WVU. For more information, please contact researchrepository@mail.wvu.edu. 


\title{
Modeling, Identification and Control of a Cold Flow Circulating Fluidized Bed
}

\author{
Rupendranath Panday \\ Thesis submitted to the \\ College of Engineering and Mineral Resources \\ at West Virginia University \\ in partial fulfillment of the requirements \\ for the degree of \\ Master of Science \\ in \\ Electrical Engineering
}

Brian D. Woerner, Ph.D., Chair

Muhammad Choudhry, Ph.D.

Lawrence J. Shadle, Ph.D.

Lane Department of Computer Science and Electrical Engineering

Morgantown, West Virginia

2008

Keywords: Cold Flow Circulating Fluidized Bed, Modeling, Identification, Control, Flow Split, Standpipe 


\title{
ABSTRACT \\ Modeling, Identification and Control of a Cold Flow Circulating Fluidized Bed
}

\author{
Rupendranath Panday
}

Circulating fluidized bed (CFB) is used extensively in petrochemical industries especially for fluid catalytic cracking, coal combustion or gasification and various other chemical processes. Modeling will help identify the sensitivity of the performance of a CFB to variation in different operating conditions and design parameters. Mathematical models also have a more practical purpose - the development of engineering and design tools which will help calculation, and design of real plants.

From the practical engineering point of view, a compact and accurate description of the dynamic behavior of the system under consideration is needed and mathematical models derived based on this requirement can be used for calculations, and design and operation of real systems. New 2-region measurement model describing the bed height in the standpipe of a cold model present at National Energy Technology Laboratory (NETL), US Department of Energy, Morgantown, WV, is formulated using the total pressure drop across the standpipe, pressure drop across the dense region and the static pressure contribution due to solids in the lean region of the standpipe.

On the other hand, modeling a system of interest directly from observed input-output data is referred to as System Identification. Identification of a NETL cold flow circulating fluidized bed (CFCFB) is carried out using a multiple model approach. Under this technique, the CFCFB is considered as a nonlinear device. From the system theoretical point of view, any nonlinear model can be decomposed into multiple sub models to cover certain operating range of a given system and these multi-models can then be combined together through some weighting functions to encompass its wide operating range. To achieve this wide operating range model validity, a white noise experiment was conducted in the CFCFB using glass beads bed material with the objective in mind that the model trained on random data sets would be sufficient enough to be utilized in other simple operating conditions. In this work, these data are used to identify CFCFB's multiple sub models and to combine them into a single nonlinear model such that solids circulation rate can be estimated from the move air flow and riser aeration fed to the device, and the total pressure drop developed across the riser at extremely different experimental conditions.

However, when a cold flow circulating fluidized bed is believed to operate linearly in a single operating regime, it is reasonable to approximate the given system by a linear model in order to predict solids flow rate. In reality, any measurable variables may be corrupted by noise and it is sensible to arrive at results that back up the initial assumption regarding the basic relationship between the variable of interest and the independent variable that is presumed during the model development phase, which would otherwise be difficult due to the use of noisy measurements. Similarly, it is also helpful to analyze the stochastic processes that corrupt the measurements, using data from one particular experiment such that future inference could be made on the errors acting on those measurements if the same machine is operated under similar condition and/or in any other industrial plants that exhibit dynamics similar to that of the laboratory-based equipment like the NETL CFCFB at that operating condition.

The present work begins with a complete black box model of a state-space description arising from the system identification and converts it into a model without any fictitious variable such that the interaction among the variables under consideration can be analyzed. Furthermore, this concept separates a state into 
stochastic and deterministic components which gives the nature of noise acting on the measurement device and rationalizes if there exists a certain relationship between independent and dependent variable. In this thesis, the state is a solids circulation rate. Independent parameters that comprise of aerations flow rates including move air flow, riser aeration and loop seal fluidization air are used to obtain deterministic component of a measured solids circulation rate. On the other hand, easily measurable dependent variables like the pressure drops across various sections of the machine are used to predict its stochastic counterpart.

A real time pressure drop model based on the Recursive Prediction Error Method (RPEM) is built to predict the split of move air flow between the standpipe and L-valve. The split estimate is of paramount importance while simulating the phenomenological model of the standpipe or in other applications, if required. Additional aeration fed across the various sections of standpipe act as the fluidization bias and their routes determination within the component may help to maintain their required level to assist in solids movement during operation while minimizing excessive flows. The path determination is also predicted using RPEM on a discrete time pressure drop model such that the user can operate them at the desired intensity according to their operating requirements.

Generally, a PID controller is not "portable", i.e., a controller designed for one plant is usually not applicable to another plant. To resolve this long-standing issue of portable controllable design, the controller scaling method can be used to control similar plants that are different only in gain and frequency scales, thus avoiding tedious control redesign. However, there are always differences in design specifications and constraints for different control problems. For the sake of simplicity and optimized controller design for each application such that an engineer get the most performance out of a given set of hardware and software, bandwidth parameter is selected as the measure of performance. To avoid the repetitive tuning of this scaling parameter based on a controller performance, adaptive algorithm is designed. Furthermore, to predict the characteristics behavior of a complex system lacking a reliable mathematical model, a recursive least squares estimation (RLS) algorithm is utilized to find autoregressive moving average (ARMA) models for single-input single-output or multi-input singleoutput case. The adaptive PID control algorithm is then tested on the benchmark NETL CFCFB plant by controlling solids circulation rate according to the reference solids flow rate obtained from the Knowlton's correlation utilizing average voidage in a moving bed condition and the move air flow.

The optimal control of solids circulation rate affecting the heat and mass transfer characteristics which in turn impacts the efficiency of various chemical processes is necessary in CFB units. An example might be the catalytic systems that recirculate catalyst in a reaction/recirculation cycle. In the case of such units in which the addition of catalyst is small and need not be steady, the main solids flow-control problem is to maintain balanced inventories of catalyst in and controlled flow from and to the reactor and regenerator. This flow of solids from an oxidizing atmosphere to a reducing one, or vice versa, usually necessitates stripping gases from the interstices of the solids as well as gases absorbed by the particles. Steam is usually used for this purpose. The point of removal of the solids from the fluidized bed is usually under a lower pressure than the point of feed introduction into the carrier gas. The pressure is higher at the bottom of the solids draw-off pipe due to the relative flow of gas counter to the solids flow. The gas may either be flowing downward more slowly than the solids or upward. The standpipe may be fluidized, or the solids may be in moving packed bed flow with no expansion. Gas is introduced at the bottom (best for group B) or at about 3-m intervals along the standpipe (best for group A). The increasing pressure causes gas inside and between the particles to be compressed. Unless aeration gas is added, the solids could defluidize and become a moving fixed bed with a lower pressure head than that of fluidized solids. Thus, this observation leads to the fact that the gas velocity in the standpipe might be the main parameter to control the solids circulation rate. 


\section{Acknowledgements}

This thesis is the result of my research experiences during the period of $2004-2008$ at the West Virginia University, the National Energy Technology Laboratory and my home at Nepal. Many people have contributed directly or indirectly to this thesis whom I would like to express my sincere gratitude. Although unfortunately I am able to mention just a few, firstly I would like to thank my promoter Dr. P. Famouri for providing a valuable research project during my stay at WVU during 2004 - 2007. I am grateful to Dr. R. Turton for his critical remarks regarding one of my conference papers.

I want to express my deepest gratitude to my research advisor, Dr. B. D. Woerner, for his guidance, encouragement and support that he provided me at the time I wanted most. Dr. M. Choudhry has encouraged me all the time; the support he provided me at the last moment at WVU is highly appreciable. I am greatly indebted to him and I will always be. I would like to thank Dr. L. J. Shadle, Dr. J. C. Ludlow and the NETL research team for conducting two test series on my request required for my thesis preparation.

Dr. Shadle's continuous thirst for new ideas keeps amazing me. Dr. J. C. Ludlow taught me how to perform research and undoubtedly, showed me the path where an engineer should in reality be able to put his (mathematical) knowledge in practice. Their excellent guidance and critical remarks to the presentation of one of my works at NETL are unforgettable. Thanks to both of you for opening the door to an incredible educational journey which I have always been looking for! I am greatly indebted to Dr. E. J. Boyle who made my entry possible to NETL and who has always supported me and actively encouraged me to finish this thesis. Again, thank you all for your support and guidance during my stay outside United States.

Three families - Cannon (Morgantown), Pant (Baltimore) and Joshi (Long Beach) - that I cannot avoid mentioning in this thesis, who helped me to survive well in a foreign land, who were always there to support me during the time full of deepest pain and sorrow and to whom I will be deeply grateful throughout my life, I would like to thank you for your unconditional support and everlasting care.

My parents, elder brother, sister-in-law, nieces and doctor deserve my greatest gratitude for their continuous support over the years; it helped me to shape my life as it is. To my doctor, friends and family I would like to say that you make my life worthwhile.

Finally, I would like to thank all of my colleagues at the West Virginia University, all of whom supported me during my research period; Yue Huang, Sudiksha Joshi and Benketesh Sharma. 
Dedicated to my family (Vidya, Rabin, Pratibha, Rasen, Irina, Sim, Soph, Murali), my doctor: Kavita and my friends: Ramesh and Suman. 


\section{Table of Contents}

$\begin{array}{lc}\text { Abstract } & \text { ii } \\ \text { Acknowledgements } & \text { iv } \\ \text { Dedication } & \mathbf{v} \\ \text { List of Figures } & \text { ix } \\ \text { List of Tables } & \text { xii } \\ \text { Abbreviations } & \text { xiii }\end{array}$

$\begin{array}{lll}\text { Chapter } 1 & \text { Introduction } & 1\end{array}$

1.1 Introduction 1

1.2 Cold Flow Circulating Fluidized Bed 3

1.3 Test Facility at NETL/US DOE 4

1.4 Literature Review 6

1.5 Contributions of the Thesis 8

$\begin{array}{lll}1.6 & \text { Outline of the Thesis } & 10\end{array}$

$\begin{array}{llr}\text { Chapter } 2 & \text { Bed Height Calculation } & 12\end{array}$

$\begin{array}{lll}2.1 & \text { Motivation } & 12\end{array}$

2.2 Packed Beds and Fluidized Beds $\quad 12$

2.3 Bed Height Calculation $\quad 12$

$\begin{array}{lll}2.4 & \varepsilon_{\mathrm{L}} \text { Values } & 16\end{array}$

$\begin{array}{lll}2.5 & \text { Results and Discussions } & 16\end{array}$

Chapter 3 Multiple Model Identification of a CFCFB 18

$\begin{array}{lll}3.1 & \text { Motivation } & 18\end{array}$

3.2 Multiple Model Identification: Basic Theory 18

3.3 Prediction Error Method 21

3.4 Estimating the Predictor Parameters 21

3.5 Results and Discussions 25 
Chapter 4 Linear System Identification of a CFCFB

4.1 Motivation 38

4.2 Linear System Identification: Basic Theory 38

4.3 Prediction Error Method 40

4.4 New System Model for the CFCFB: Grey Box Model 41

4.4.a Model Order Reduction when $\mathrm{n}>\ell \quad 42$

4.5 Results and Discussions 44

4.5.a Validation of the Model 45

4.5.b Deterministic Analysis of Solids Circulation Rate 48

4.5.c Stochastic Analysis of Solids Circulation Rate 50

4.6 Procedure 54

4.7 Limitations of Present Approach 55

Chapter 5 Air Flow Split and Route Estimation in a CFCFB 56

5.1 Motivation 56

5.2 Pressure Drop across the Bottom of the Standpipe 57

5.3 Recursive Prediction Error Method 59

5.4 Pressure Drop across the L-valve 61

5.5 Route Estimation for Aerations Fed Across the Standpipe 63

5.6 Results and Discussions 64

5.6.a Split Estimation 64

5.6.b Route Estimation 67

$\begin{array}{lll}\text { Chapter } 6 & \text { Adaptive PID Control of a CFCFB }\end{array}$

$\begin{array}{lll}6.1 & \text { Motivation } & 71\end{array}$

6.2 An Adaptive PID Control Algorithm 71

$\begin{array}{lll}6.3 & \omega_{\mathrm{c}} \text { Estimation } & 73\end{array}$

6.4 Summary of Adaptive PID Control Algorithm 74

6.5 Adaptive PID Control of a CFCFB 75 
Chapter 7 Conclusions and Recommendations

$\begin{array}{ll}\text { Appendix } & 81\end{array}$

$\begin{array}{ll}\text { A1 Characteristics of Solid Particles Used } & 81\end{array}$

A1.1 General Definitions $\quad 81$

A1.2 Particle Classification for Cork and Glass Beads 81

A2 Components of a NETL Cold Flow Circulating Fluidized Bed 83

$\begin{array}{lll}\text { A2.1 Riser } & 83\end{array}$

A2.2 Cross Over 83

A2.3 Gas-Solids Separator $\quad 84$

A2.4 Standpipe 84

A2.5 Solids Recycle Systems 84

A3 Pressure Balance Mechanism in a NETL CFCFB 85

A4 Solids Flow Rate Measurement in a NETL CFCFB 86

$\begin{array}{lr}\text { Notations } & 88\end{array}$

$\begin{array}{ll}\text { References } & 94\end{array}$ 


\section{List of Figures}

Figure (1.1): Schematic of a cold flow circulating fluidized bed present at NETL, US Department of Energy, Morgantown, WV

Figure (2.1): Schematic of a cold flow circulating fluidized bed. Also shown are the two region model of the standpipe and a plot of pressure profile in the standpipe

Figure (2.2): Comparison of measured and calculated bed height around $8.2 \mathrm{~m}$

Figure (2.3): Comparison of measured and calculated bed height greater than $8.2 \mathrm{~m}$

Figure (3.1): Block diagram for prediction of solids circulation rate using MMI

Figure (3.2): Block diagram for prediction of solids circulation rate using N4SID

Figure (3.3): $\quad$ BED MATERIAL - GLASS BEADS. Training data set that comprises of riser aeration and move air varied randomly one at a time and the developed pressure drop across the riser as the inputs (taken in September 2006). Also shown is the prediction of solids circulation rate with the predictors defined by MMI and N4SID

Figure (3.4): BED MATERIAL - GLASS BEADS. Validation data set that comprises of riser aeration, modulated move air flow and the developed pressure drop across the riser as the inputs (taken in September 2006). Also shown is the prediction of solids circulation rate with the predictors defined by MMI and N4SID

Figure (3.5): $\quad$ BED MATERIAL - GLASS BEADS. Validation data set that comprises of random riser aeration and move air flow, both varied simultaneously and the developed pressure drop across the riser as the inputs (taken in October 2006). Also shown is the prediction of solids circulation rate with the predictors defined by MMI and N4SID

Figure (3.6): BED MATERIAL - CORK. Validation data set that comprises of riser aeration modulated move air flow and the developed pressure drop across the riser as the inputs (taken in December 2001). Also shown is the prediction of solids circulation rate with the predictors defined by MMI and N4SID

Figure (4.1): Experimental condition - K39. Comparison of measured and estimated SCR

Figure (4.2): Experimental condition - K39. Comparison of deterministic measured and estimated solids circulation rate

Figure (4.3): Experimental condition - K39. Comparison of deterministic estimated solids circulation rate and move air flow to verify linear relationship between them

Figure (4.4): Experimental condition - K39. Comparison of stochastic estimated solids circulation rate and measured SCR.

Figure (4.5): Experimental condition - K39. Histogram for stochastic measured solids circulation rate 
Figure (4.6): Experimental condition - K39. Histogram for stochastic estimated solids circulation rate

Figure (4.7): Experimental condition - K39. Comparison of probability density function of stochastic measured and estimated solids circulation rate

Figure (4.8): Experimental condition - K39. Plot of pressure drops across various sections of the CFCFB vs. deterministic solids circulation rate to indicate all pressure drops lead SCR except $\Delta P_{L P S}$

Figure (4.9): Experimental condition - K39. Normality plot to show stochastic measured solids circulation rate indeed follows a normal distribution

Figure (4.10): Experimental condition - K39. Normality plot to show stochastic estimated solids circulation rate indeed follows a normal distribution

Figure (5.1): Illustration of a split of move air flow between the standpipe and L-valve of a cold flow circulating fluidized bed

Figure (5.2): A comparison plot of original move air and its split between the standpipe and the Lvalve

Figure (5.3): A comparison plot of measured and estimated bottom standpipe pressure drop based on more than $50 \%$ move air flow

Figure (5.4): A comparison plot of measured and estimated L-valve pressure drop based on less than $50 \%$ move air flow

Figure (5.5): A plot of move air split parameter per second at the location where the spiral vane is placed within the standpipe

Figure (5.6): A comparison plot of additional aeration flows and their routes within the standpipe 70

Figure (5.7): A comparison plot of measured and estimated pressure drop at the spiral location based on aerations including move air flow splits

Figure (6.1): Common controller design scenario

Figure (6.2): The cold flow circulating fluidized bed test plant. Only part of it required for control is shown.

Figure (6.3): $\quad$ Measured solids circulation rate (SCR)

Figure (6.4): $\quad$ Reference SCR and controlled SCR

Figure (6.5): $\quad$ Error between reference SCR and controlled SCR

Figure (A1.1): Powder classification developed by Geldart

Figure (A1.2): Geldart's classification for the particulate materials used in the thesis 
Figure (A3.1): The principle of working a CFCFB can be explained by the example of moving water in a loop by aeration

Figure (A3.2): The pressure balance around the CFCFB loop. The dotted lines represent the pressure profile at a higher circulation rate

Figure (A4.1): NETL spiral device designed by James Christopher Ludlow 


\section{List of Tables}

Table (3.1): Characteristics of glass beads used for obtaining identification data obtained in September, 2006

Table (3.2): Experimental condition at which move air flow was modulated. The experiment was performed with glass beads material in September, 2006

Table (3.3): $\quad$ BED MATERIAL - GLASS BEADS. Number of models in a multiple model approach and the corresponding model fits for the validation data set where move air flow was modulated. Model fit for linear time invariant system obtained with N4SID algorithm is also shown

Table (3.4): $\quad$ BED MATERIAL - GLASS BEADS. Number of models in a multiple model approach and the corresponding model fits for the validation data set where both riser aeration and move air flow were varied randomly at the same time. Model fit for linear time invariant system obtained with N4SID algorithm is also shown

Table (3.5): Experimental condition at which move air flow was modulated. The experiment was performed with cork material in December, 2001

Table (3.6): $\quad$ BED MATERIAL - CORK. Number of models in a multiple model approach and the corresponding model fits for the validation data set where move air flow was modulated. Model fit for linear time invariant system obtained with N4SID algorithm is also shown

Table (4.1): Comparison of the modelfits between the estimated solids circulation rate obtained from Eq. (4.5) and those from the combined stochastic and deterministic solids circulation rate from the grey box model 


\section{Abbreviations}

$\begin{array}{ll}\text { CFB } & \text { Circulating Fluidized Bed } \\ \text { FCC } & \text { Fluidized Catalytic Cracking } \\ \text { FBC } & \text { Fluidized Bed Combustor } \\ \text { MWh } & \text { Mega-Watt hour } \\ \text { CFBC } & \text { Circulating Fluidized Bed Combustion } \\ \text { CFCFB } & \text { Cold Flow Circulating Fluidized Bed } \\ \text { NETL } & \text { National Energy Technology Laboratory } \\ \text { DOE } & \text { Department } \bigcirc \text { f Energy } \\ \text { SCR } & \text { Solids Circulation Rate } \\ \text { EKF } & \text { Extended Kalman Filter } \\ \text { MATLAB } & \text { MATrix LABoratory } \\ \text { SITB } & \text { System Identification Toolbox } \\ \text { MIMO } & \text { Multi-Input Multi- } \bigcirc \text { utput } \\ \text { MMI } & \text { Multiple Model Identification } \\ \text { PEM } & \text { Prediction Error Method } \\ \text { N4SID } & \text { Numerical SubSpace for State Space Description } \\ \text { RPEM } & \text { Recursive Prediction Eror Method } \\ \text { PID } & \text { Proportional-Integral-Derivative } \\ \text { RLS } & \text { Recursive Least Squares } \\ \text { FF } & \text { Forgetting Factor } \\ \text { LTI } & \text { Linear Time Invariant } \\ \text { LTV } & \text { Linear Time Varying } \\ & \end{array}$




\section{Chapter 1}

\section{Introduction}

\subsection{Introduction}

Circulating fluidized bed (CFB) technology has been applied to power generation, catalytic cracking, coal combustion and other petroleum refining operations as well as various other chemical processes. It is a popular device used to react a granular solid with a fluid because of the high solid-fluid contacting efficiency. During the combustion process, the solids particles are suspended in the upwardly flowing gas and they are mixed in a turbulent fashion that provides more effective chemical reactions and heat transfer. Other advantages of circulating fluidized beds include excellent suitability for large-scale operations, better interfacial contacting and reduced back mixing. They can burn a wide variety of fuels such as coal, waste coal, anthracite, lignite, petroleum coke and agricultural waste with low heating value $(>1500 \mathrm{kcal} / \mathrm{kg})$, high moisture content $(<55 \%)$, and high ash content $(<60 \%)$, Kavidass et al. [1]. Add-on equipment is not necessary to use in a fluidized bed boiler to absorb sulphur dioxide $\left(\mathrm{SO}_{2}\right)$ generated during combustion in its furnace because of its low combustion temperature that is usually in the range of 800 to $900{ }^{\circ} \mathrm{C}$. Low emission of nitrous oxide $\left(\mathrm{NO}_{2}\right)$ during combustion is another attractive feature of CFB boilers.

Fluidization is a process whereby a bed of solids particles is transformed into something closely resembling a liquid. The upward flow of a fluid through a bed of particles gives rise to fixed bed at low flow rates, but if the velocity is sufficient to exert a force on the particles that exactly counteracts their weight, the particles will be freely supported in the fluid to give rise to a fluidized bed. In this way, the bed acquires fluid like properties, free to flow and deform, with the particles able to move relatively freely with respect to one another, Gibilaro [2].

A major application of fluidized technology is to be found in the catalytic-cracking reactor, which lies at the heart of the petroleum refining process. Fluid catalytic cracking (FCC) reactor is one of the important processes in petrochemical industry where heavy oil is injected in gas-solid risers. The FCC riser reactor converts heavy hydrocarbon petroleum fractions into a slate of more usable products such as gasoline, middle distillates and light olefins.

In energy companies, the combustion in a bubbling fluidized bed has become attractive when compared to the conventional combustion technologies, to burn solids, liquid and gas fuels as well as waste fuel and biomass consumption. This is because fluidized bed combustor (FBC) boilers are markedly superior to all

other combustion technologies in burning low quality coals, biomass and other waste fuels, as some of these fuels can be burned only in FBC boilers. According to Wiegan [3], Gaglia et. al. [4], Blauw et. al. [5] and Renz [6], in the range of $50 \mathrm{MWh}$ and over, $\mathrm{FBC}$ boilers are associated with lower unit energy production costs than the stationary, bubbling FBC boilers. They are cheaper by $10-15 \%$ than pulverized coal combustion boilers equipped with flue gas desulphurization.

Small and medium power boilers (up to $50 \mathrm{MWh}$ ), grate firing boilers and boilers burning liquid or solid fuels, which are nonoperational due to either shortage of liquid fuels or their failure to comply with the increasingly strict regulations on environmental protection, or which are uneconomical to operate, can be altered more easily to FBC technology. However, reconstruction of large boilers firing liquid fuels is not technically or economically justified if bubbling FBC is considered. In such a situation, reconstruction for 
pulverized coal combustion or circulating fluidized bed combustion (CFBC) is more acceptable. If the strict environmental protection regulations must also be fulfilled, CFBC is markedly superior. Therefore, when the utilization of different fuels is required, or if the previous mine has been exhausted, or cheaper fuels are available on the market, or if compliance with strict $\mathrm{SO}_{2}$ and $\mathrm{NO}_{2}$ emissions regulation are required, revitalization or reconstruction of old pulverized coal combustion boilers into circulating fluidized bed boilers becomes a more acceptable solution Basu et. al. [7], Tollet et. al. [8], Basu [9].

The use of fluidized beds in cement industries is another promising area of application, especially in the context of greenhouse gas reduction. Two raw materials, limestone and high-ash coal, are prepared separately by grinding and then fed to a CFB boiler at around $8508{ }^{\circ} \mathrm{C}$ for simultaneous precalcining of the limestone and combustion of high-ash coal with relatively high efficiencies, Reh [10]. Hot bed materials, which have the composition of clinker, discharge directly into a relatively short rotary kiln, where clinker formation occurs mainly under exothermic conditions, Squires [11]. Only a small amount of additional fuel (coal) has to be fired in the kiln to raise its temperature to that required for clinkering. Off-gases of the kiln and the adjacent cooler are sent as preheated secondary air into the CFB boiler.

The flue gas from the boiler furnace passes through its back-pass (convective section) and particulate collection device. Fly-ash captured by the particulate collector is sent to the clinkering machine for cement production, while steam generated in the boiler drives a turbine to produce electricity. The condensed steam can be used for space heating. Thus the process offers the trigeneration potential of producing cement clinker, electricity, and heat with low carbon dioxide and nitrous oxide emissions.

The direct substitution of ash for the clay component in cement saves the mining of clay and avoids disposal of ash completely. The investment cost is generally lower than that for two separate plants and heat utilization efficiency is very high. This option is useful particularly to countries with high cement and electricity demand and large reserve of low-grade coal.

Gas fluidized beds are also widely used as chemical reactors. Liquid-fluidized beds are employed extensively in water treatment, minerals processing and fermentation technology. Generally, the application of CFBs, established and potential are boundless. In summary, the principal application of gas-solid fluidization is in the following industries:

1. Energy conversion

2. Petro-chemical processing

3. Mineral processing

4. Chemical and pharmaceutical

5. Physical processing

Some major applications in each of the above areas are given below:

1. Energy conversion

- Steam generation

- Gasification

- Incineration

2. Petro-chemical processes

- Fluid catalytic cracking units

- Fischer-Tropsch Synthesis

3. Mineral Processing

- Calcination of alumina

- Roasting of ores 
- Prereduction of iron ores

- Precalcination for cement manufacture

4. Chemical and Pharmaceutical

- Phthalic anhydride from naphthalene

- Decomposition of sulfate, chloride

- Butane oxidation to maleic anhydrite

- Methanol to olefins conversion

5. Physical Processing

- Drying

- Coating of particles

- Heat exchanger and flue gas cleaning

- Heat treatment

Despite being embraced with such tremendous potentials to serve mankind, unfortunately, the circulating fluidized bed is not an exceptional creation in Mother Nature ruled out by disadvantages. Higher capital cost, and operating and maintenance expenses might be the primary limiting factors for developing nations, automation system vendors, universities, research centers, and the process industries to adopt or to continually strive to invent new and better methods in order to keep processes under control. Others including the operational issues might be the added complexity in designing and operating recirculating loop, or decreased suspension-to-wall heat transfer coefficients for given particles.

Regardless of all these drawbacks that are insignificant as compared to its enormous advantages benefiting humans, the fluidized bed technology is still a bright area for future research to dwell upon in order to improve and provide substantial gains in process industries, power generation companies, coal combustion technologies, petrochemical industries, pharmaceutical industries and physical processes.

\subsection{Cold Flow Circulating Fluidized Bed}

Research into the mechanisms of the fluidization process falls largely into two distinct categories: applied research, involving actual process plant or, more usually, laboratory units that seek to mimic the particular feature of the process plant that is the subject of study; and theoretical analysis, rooted in the rigorous framework of multiphase fluid mechanics, Gilibaro [2].

The construction and commissioning of large-scale process equipment, often involving high operating temperatures and pressures is extremely costly, so that any uncertainty at the design stage regarding the fluidization quality that will result in the completed plant represents a major cause of concern.

One way of tackling the problem is to build a model bed in which the fluidization quality of the proposed model can be simulated and studied. The cold model (cold flow circulating fluidized bed (CFCFB)) was built at National Energy Technology Laboratory (NETL), US Department of Energy (DOE), Morgantown, WV, where the particles, fluid and operating conditions are chosen to ensure equivalence to the final plant. Only fluidization characteristics are considered, so that the model can be operated without the heat transfer and chemical reaction processes required of the envisaged commercial unit; it may therefore be operated under ambient conditions of temperature and pressure (or perhaps under somewhat elevated pressure) and constructed cheaply perhaps using transparent material through which the behavior may be directly observed. The scaling criteria can then be used to map the fluidization quality of a cold model to that of the industrial system.

Due to the large scale of applications in different industries using this technology, small increases in overall system efficiency, through both improved raw material usage and utility consumption, would 
yield substantial savings, Park et. al. [12]. Therefore, optimization and control for CFB applications is very important and requires an accurate, real time model to describe and quantify the process, Huang [13]. However, an entire mathematical model is not available to fully represent the dynamics of a circulating fluidized bed. Because an adequate model would enhance the ability to operate and control CFBs and would significantly increase their efficiency, the modeling effort was undertaken to this end in order to analyze and predict the behavior of the cold flow circulating fluidized bed located at NETL.

\subsection{Test Facility at NETL/US DOE}

All these years the test unit present at NETL has been a center of research for CFBs communities, Chemical Engineering Departments and/or Control communities of many educational institutions through out United States. The prototype model consists of a riser, a gas/solid separator, a standpipe, a nonmechanical valve (shown in Fig. (1.1)) and other advanced measuring instruments. The riser is $15.45 \mathrm{~m}$ high and $0.305 \mathrm{~m}$ in diameter and it has several pressure sensors along its length. The main aeration (riser aeration) for the riser is at the bottom. Similarly, a $13.41 \mathrm{~m}$ high and $0.253 \mathrm{~m}$ wide standpipe contains eight pressure taps and four aeration points to maintain fluidization. During operation, solids flow up the riser due to the application of high airflow at the bottom. As the flow exits the top of the riser, the gas/solid separator, the cyclone, separates the gas from the solids. Gas is vented out while the solids fall back into the standpipe forming a dense bed. The non-mechanical loop seal valve (or an L-valve) connecting the bottom of the standpipe and riser allows the control of the solids circulation rate (SCR). Solids are fed from the bottom of the standpipe to the riser through the non-mechanical valve thus completing the CFB loop.

One of the variables that connects the dynamics of the riser with the standpipe is the solids flow rate. Its knowledge is essential to the control and improved performance of a circulating fluidized bed system. As the need to crack more and heavier hydrocarbon at even-shorter contact times grows in FCC riser reactor, oil droplet vaporization may become rate limiting. Droplet size distribution, particle characteristics (solids flux, volume fraction, diameter, heat capacity, and density), contact between the particles and the drops and the characterization of fluidizing medium determine the quality of overall process.

Momentum transfer, heat transfer, catalytic cracking reaction and droplet vaporization are interrelated and occur simultaneously in the commercial FCC riser reactor. To predict the accurate behavior of the riser reactor all these processes need to be modeled.

Similarly, with the advent of a concern for more energy, circulating fluidized bed technology has become attractive and the increased introduction of coal is imperative. Large volumes of solid material must be moved at one or many stages in the conversion of coal to energy. The rate at which the solids can be circulated has significant effects on the operability of coal combustion systems. A system that circulates badly is difficult to operate and may be run at a lower rate than desired, having an adverse effect on yields and product selectivities.

A twisted fiberglass vane installed in the packed bed portion of the standpipe of a cold model at NETL measures the value of solids circulation rate. Data is usually collected at $1 \mathrm{~Hz}$ for this variable.

Bed height is another factor that is important for the successful operation of a circulating fluidized bed unit. High performance assessment and prevention of high temperature operation are all attributed to the optimal control of a bed height level in the standpipe. This is because the measurement of too high bed height signifies that the system is running at high pressures that may result in the costly operation of the unit. Similarly, low bed height indicates that the system is not properly fluidized. A portion of the NETL CFCFB standpipe wall is made of clear acrylic for visual observation so that the bed height can be observed and recorded. The bed height is usually recorded at 10 to $15 \mathrm{~Hz}$. 


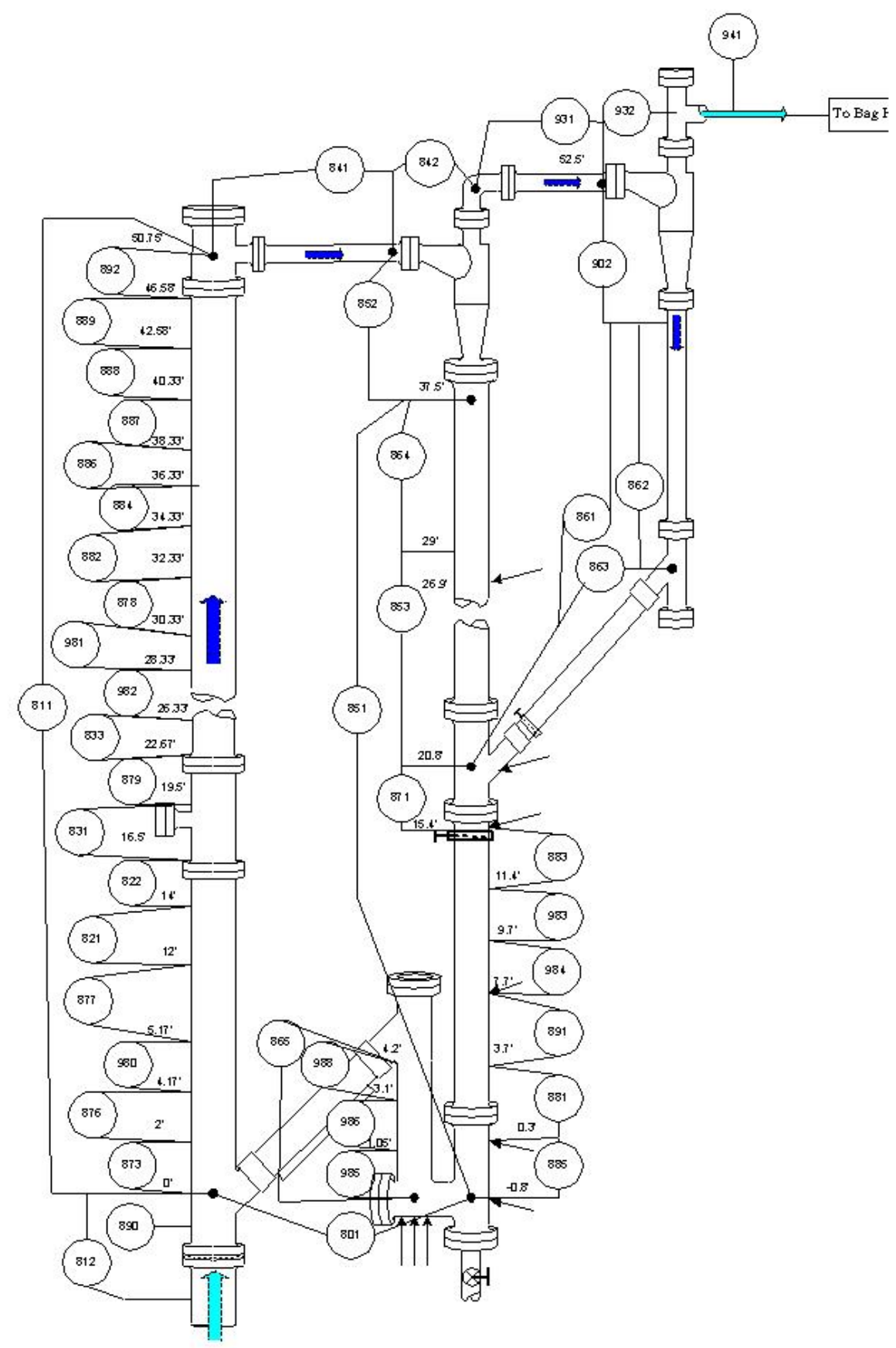

Figure (1.1): Schematic of a cold flow circulating fluidized bed present at NETL, US Department of Energy, Morgantown, WV. 
Third, pressure fluctuations are the commonly measured parameters in studies of a cold flow circulating fluidized bed. A pressure transducer, with a pressure range of $0-8 \mathrm{kPa}$ and an accuracy of $\pm 0.45 \mathrm{~Pa}$, attached to a pressure tap, is used to measure the pressure differential characteristic of the fluidized bed at one second sampling rate.

Finally, various control strategies are used to regulate pressure around the circuit by manipulation of either riser transport flow or standpipe aeration flows or loop seal (L-valve) aerations.

The emphasis of some of the work that has been carried out prior to this date was not on developing accurate flow model for overall process of a CFCFB but was on developing a framework that could predict well the solids circulation which affects the mass and heat transfer characteristics that further impact the efficiency of various chemical processes, besides bed height. The purpose of this test facility is to generate well-defined test data for model development and to verify it against the similar operating conditions carried out at different times. Other research includes the development of a measuring device for solids circulation rate, the identification of various operating regimes of the CFCFB, the transient analysis of a riser using a specific experimental procedure, and the optimal control of a device. All these works related to a NETL CFCFB are discussed in the following section.

\subsection{Literature Review}

Over the past years, a one-dimensional dynamic model of the standpipe was developed by Sams [14] for state estimation. In this model, gas void fraction is treated as a state variable. It is based on the continuity equation and a modified Richardson-Zaki Correlation to relate the slip velocity between the gas and solids to the void fraction in the fluidization condition. An extended Kalman filter (EKF) was developed in Shim et. al. [15] to estimate the state by comparing the pressure measurements to those estimated through a truncated Ergun equation.

For some time varying case in which EKF was not performing well, $\mathrm{H}_{\infty}$ estimation method was successfully applied in Jalali et al. [16]. This method used the same algorithms and approximations of the system model as those of the EKF. However, the measurement and process noise were assumed to be different than those of EKF which assumes them to be white Gaussian noise. The state estimation problem was then reduced to minimizing a single cost function parameter unlike EKF.

To estimate the void fraction utilizing a one dimensional dynamic model of the standpipe, the solids superficial velocity is required. In [12] and Park et. al. [17], the solids circulation rate (SCR) estimator was developed using a Kalman filter on the system derived from the system identification toolbox (SITB) in the MATLAB environment through the subspace algorithm, N4SID.

In the absence of process and measurement noise, move air flow, riser aeration and total pressure drop across the riser were chosen as the inputs. This model required eight pressure drops measurements across the standpipe to estimate the SCR. The SCR was then utilized to determine solids superficial velocity required to estimate void fraction and pressure profile using a sliding mode estimator. The solids circulation rate and void fraction were estimated with only four pressure drops across the standpipe by Rupen et al. [18].

In [18], it was believed that in industrial applications, it would be economical to keep the number of pressure tap measurements as small as possible to determine solids circulation rate and void fraction. Certainly, each transmitter that is used at NETL costs about $\$ 2,000$ and there are many installed on the unit. Since it is a research facility and having all of these pressure measurements is important to understand the fluid mechanics going on within the unit, the smallest number of pressure variables offers a least significant financial benefits at such a set up. However, in an industrial unit, when one builds a 
production facility (as opposed to a research facility) they do not want to add unnecessary instrumentation. In a hot reacting transport reactor unit, there are added expenses for engineering the ports, purging, and then diluting the product stream. The process engineers want to keep those costs to a manageable level and hence, they want to minimize instrumentation ports and devices to only those necessary. The rational behind choosing only two pressure drops in the dense region of the standpipe was based on the proposition of a linear relationship between the pressure profile and the height of the standpipe in the lower region especially observed with the analyzed cork data set and the belief that other pressure drops in the dense region of the standpipe could be satisfactorily determined from these two dense phase pressure drop measurements and the respective heights using the linear model.

In [12] and [17], the formulae for bed height calculation based on a two region model concept were derived. A linear controller was developed to control the SCR and bed height. For testing this linear controller, an entire mathematical model of the CFCFB system was required and the neural network based model that was obtained with available sets of experimental data, was incorporated as a complete model of the CFCFB.

On the other hand, different approach to model and control CFB using neural networks was carried out in Davari et al [19]. The solids flow rate was modeled as a function of the differential pressures in the standpipe section and the flow rate of the aeration utilizing a multi-input multi-output (MIMO) neural network model, and a neural network controller was developed to manipulate the mass flow rate by varying the aeration.

In [13], the transient behavior of large CFB units was modeled using cork as the fluidizing material and air at ambient conditions. A tank in series model and a cluster flow model were used to model the riser. The solids flow rate and voidage in the dense phase of the standpipe were predicted using the model based on Ergun equation. To obtain the integrated CFB model, the pressure balance analysis was utilized to simulate the CFCFB in real time under transient conditions.

Many other research projects were performed related to the CFCFB by a NETL research team. The development of a spiral device to measure the solids circulation rate was presented and explained in Ludlow et al [20]. The overall solids circulation rate was calculated using the speed of rotation of the twisted vane, the bulk density of solids, and the cross-sectional area of the standpipe when the passage of solids through the spiral vane forced it to rotate during operation.

In Lawson et al. [21], the hydrodynamics of each component leg in the circulating fluid bed was characterized using polyvinyl chloride particles to facilitate control of integrated circulating loop. In addition, both open-loop and close-loop analysis techniques were investigated. The process variables were defined and dependencies among them were characterized experimentally. Control techniques for uncoupling process variables around the circulating loop were demonstrated.

In Shadle et al.[22], the flow regimes were studied from the homogenous dilute-phase flow regime to dense phase fluidization flow regime employing coarse cork particles. Flow instabilities in riser pressure fluctuations and solids circulation rates when operating in the intermediate, turbulent and fast fluidization regimes were discussed. Operating flow regime was evaluated and axial voidage profile along the riser for different flow regimes were characterized as a function of gas velocities and mass fluxes. Saturation carrying capacities were determined as a function of riser velocity using the solids cut off method.

Similarly, in Shadle et al. [23], simple and rapid dynamic tests were used to evaluate fluid dynamic behavior of granular materials with particle densities ranging from 189 to $2500 \mathrm{Kg} / \mathrm{m}^{3}$ and mean sizes from 61 to $812 \mu \mathrm{m}$ in the transport regime. Standpipe aeration was ramped to obtain maximum solids loading of the riser. The solids flow was then abruptly halted leading to a riser pressure drop. The 
associated solids flux was estimated for each time step from the changes in the pressure drop per unit time during riser emptying transient. Limitations for accurately estimating the related steady state condition were identified in each operating regime. Experimental results required to obtain accurate unbiased results were discussed including effects of bed materials, gas pressure and particle size distribution.

\subsection{Contributions of the Thesis}

A NETL cold flow circulating fluidized bed is a highly complex system characterized by a large number of components that are strongly coupled and have a large number of non-measurable unknowns internal to the system. Modeling and control of such a complicated system is one of the more challenging areas of system theory.

In [12], a formula to calculate bed height was derived based on a 2- region model concept comprising of two homogenous regions. It utilizes the total pressure drop across the standpipe and the pressure drops across the dense and lean region of the standpipe. The difficulty with this 2-region model formula is sometimes the bed in the standpipe is high enough to cover the lower tap defining the pressure drop (between $8.2 \mathrm{~m}$ and top of the standpipe) in the lean region. This problem can be avoided if the simplification that the pressure drop at the top of the bed is considered same as the pressure drop at the top of the standpipe is used. A modified algorithm was developed in [17], where such a problem did not exist. However, the void fraction measurements that are not available from the cold unit at NETL are not known exactly for employing in this modified algorithm. The lean phase void fraction has to be calculated either from the previous pressure drop when the bed height is less than $8.2 \mathrm{~m}$ or is assumed to be of a constant value and the dense pressure drop in the standpipe is required to calculate void fraction in that region. A new formula has been developed in Chapter 2 that involves only lean phase void fraction term rather than three. The choice of this unknown quantity that was found to be in the range 0.98 and 1 in [18] giving satisfactory results with different experimental data set is used in the new formula.

The third chapter focuses on the prediction of the solids circulation rate as a function of riser aeration, move air flow and total riser pressure drop based on a multiple model identification algorithm (MMI) derived by Verdult et. al.[24]. It identifies composite state space models using a projected gradient search method from the input and output measurements taken from the given system. Within this multiple model domain, the CFCFB is considered as a nonlinear black box dynamic system. A white noise experiment was conducted using glass beads as a bed material and by varying move air flow and riser aeration uniformly one at a time to obtain a data set that is used for estimating the predictor model parameters. The resulting model is tested on fresh data sets to validate the prediction of solids circulation rate and compared with observed measurements of SCR made at different experimental conditions in addition to the measurements taken with the cork bed material. On the other hand, the same system is modeled using the global linear state space predictor model estimated by the N4SID algorithm present in the system identification toolbox in the MATLAB environment. The output response obtained from both these models is compared in terms of the model fit and the results are shown; the results from the nonlinear predictor model outperform those from the linear model in all the cases.

In [12], and [17], the initial system was determined from the N4SID algorithm without considering measurement and process noise acting on the CFCFB. Then, using the output-to-state transformation, the Kalman filter was applied to estimate the deterministic states. Such concept concludes that the CFCFB was noise free when its initial model was determined and the same system was acted upon by the noises when the Kalman filter was used to estimate its state variables. Moreover, Kalman filter is robust in the statistical sense in the presence of noises acting on the system and hence, its use in the state estimation seems ambiguous in [12], and [17]. Most probably the simulation results in these works were due to the system identification rather than Kalman filter. 
Moreover, the use of N4SID algorithm to determine system matrices as in [12], and [17] results in biased parameter estimates because all these experiments in the CFCFB at NETL are carried out in the closed loop in the presence of the PID controllers [21] and the N4SID algorithm present in the SITB is implemented for the open loop case. Since this is not the case with the prediction-error-method (PEM), Ljung [25], while the system is operating under closed loop, the PEM is used in place of the N4SID algorithm in Chapter 4, although its characteristics are much superior to those of the PEM Overschee et. al. [26]. If one is interested to use N4SID for the closed loop system, then the closed loop N4SID identification algorithm derived as in Overschee et. al.[27] should be employed.

In Chapter 4, the noise model is derived considering process and measurement noises acting on the cold flow circulating fluidized bed with a cork particulate material. The outputs of the initial model are the total pressure drop across the riser, the pressure drop across the crossover, the pressure drop across the primary cyclone, the total pressure drop across the standpipe, the pressure drop across the loop seal and the solids circulation rate. The stochastic estimate of solids circulation rate is determined from the noise model using the stochastic pressure drop estimates. The deterministic estimate is obtained through the inputs taken as move air flow, riser aeration and loop seal fluidization air that are all independent variables of the given set up and under the control of the user. The theory has been developed to convert a complete black-box model to a grey box model through the output-to-state transformation such that both the models of the CFCFB consists of all these output variables as the states of the system and only pressure drops across the system as the output measurements. Thus, the final models do not include any fictitious terms and they are defined only in terms of physical parameters of the given system. Both components of solids circulation rate are separately analyzed. The combined solids circulation rate response of both the noise model and deterministic model is compared with the validation data set of this state variable in terms of modelfit and the results are shown.

Furthermore, it has always been a concern in CFB community and Control community whether a CFB is a linear or a nonlinear device. With the present sets of experiments that are conducted under dilute transport regime, [13], with cork bed material, the current work rationalizes under the careful explanation of the cause of move air flow on pressure drops and solids circulation rate, that the CFB necessarily operates in a linear fashion under this regime. In other regimes of operation, perhaps this is not the case. It is also discussed and justified that the measurement errors experienced during pressure taps measurements and spiral vane measurements follow a normal distribution.

All the previous approaches using system identification based on linear/nonlinear system theory [12], [13], [18], and Rupen et. al.[28] or on neural network theory [19] to model the fluidized bed column use the dependent variables such as the pressure drops across the various sections of the CFCFB as the inputs to the system to determine system parameters. In the present case, the aerations that are independent variables and under the control of the user are used for this purpose. The advantage of using the independent variables such as move air flow and other aerations flows for identification is that these can be independently controlled and do not depend upon other parameter settings. The pressure drops are responses and as such may be influenced by the total inventory of solids in the unit and other things which may not be easily known in a hot unit. An example might be the use of pressure drops to measure standpipe bed height. For some modes of operation, the ratio of an incremental pressure drop to the overall pressure drop might be used to estimate a bed height; however, if the inventory is below the incremental pressure taps chosen for the measurement, then the particular response is not a representative of the pressure drop in the bed and will lead to unrealistic results. The response variables can be used for these purposes, but the constraints must be well understood. On the other hand, the physical phenomena taking place inside the CFCFB during operation is taken into account to choose the output for the initial black box modeling of a present model. This helps to determine the stochastic response of solids circulation rate from those of pressure drops. 
There are other modeling efforts where the prediction of some of the dependent variables from independent variables is desired because at other facilities, the ability to measure those dependent variables does not exist. The solids circulation rate is a good example. At the CFCFB facility in NETL, there is the spiral [20] which continuously measures circulation rate but to implement such an instrument in a hot pressurized unit would be very difficult or perhaps even impossible. There it is desirable to have a model which would allow the estimation of the circulation using independent inputs or dependent variables that are easily measurable. The model developed in this chapter pursue a goal of calculating the solids circulation rate which is not easily measured or as a substitute for making its measurements when the CFCFB operates under dilute transport regimes.

In Chapter 5, a novel approach is used for the online estimation of the split of a move air flow between the standpipe and the L-valve of a cold flow circulating fluidized bed (CFCFB). The treatment technique using the Recursive Prediction Error Method (RPEM) is developed to create a discrete time model describing the pressure drop at the bottom of the standpipe at the current time as a function of the product of the average mass flux and a split of move air flow and the bottom pressure drop at the previous time instant. The comparison of measured and estimated L-valve pressure drop as a function of the L-valve aeration and the remnant move air flow is used to verify if the split is correct.

On the other hand, it is a common scenario within the standpipe fed with additional aerations across various sections to assist solids movement, that these air flow either in upward or downward direction. Extra job is then to identify the route of these aerations within the standpipe during operation of the CFCFB. A procedure of estimating these aerations path is designed in this chapter.

Referring to the control action, controller scaling can be used to frequency-scale an existing PID controller for a large class of plants which eliminates the repetitive controller tuning process for plants that differ mainly in gain and bandwidth, Gao [29]. Controller parameterization makes the controller parameters a function of a single variable, the loop-gain bandwidth, and greatly simplifies the tuning process. The controller is scaled according to the gain and frequency scales of the given plant transfer function and the bandwidth parameter whose initial value is set based on the bandwidth requirement from the transient response. This single parameter is required to be gradually adjusted according to a controller performance.

However, if the explicit transfer function of a given system is not known, the gain and frequency scales should be determined from an empirical model transfer function obtained from the System Identification procedure. In Chapter 6, we propose a new technique based on the theory of Recursive Least Squares (RLS) Estimation that allows determining the bandwidth parameter as well as the closed loop model parameters online. As the technique is recursive by nature, it further avoids the adjustment of a bandwidth parameter in accordance with the controller performance.

\subsection{Outline of the Thesis}

The thesis is a compilation of results that have been published or might be published elsewhere in different form.

R. Panday, B. D. Woerner, J. C. Ludlow, L. J. Shadle, and E. J. Boyle, "Linear System Identification of a Cold Flow Circulating Fluidized Bed", Journal of Process Mechanical Engineering, London, UK, June, 2008.

R. Panday, P. Famouri, R. Turton, B. D. Woerner, J. C. Ludlow, L. J. Shadle, and E. J. Boyle, "Multiple Model Identification of a Cold Flow Circulating Fluidized Bed", CFB-08, Hamburg, Germany, May, 2008. 
R. Panday, P. Famouri, R. Turton, and E. J. Boyle, "Identification of a Standpipe of CFCFB with a Minimal Number of Pressure Drops”, CCCT-06, Florida, USA, July, 2006.

R. Panday, B. D. Woerner, J. C. Ludlow, L. J. Shadle, and E. J. Boyle, “Air Flow Split Route Estimation in a Cold Flow Circulation Fluidized Bed", Powder Technology, USA, To be sent.

R. Panday, B. D. Woerner, J. C. Ludlow, L. J. Shadle, T. Woerstell and E. J. Boyle, "Adaptive PID Control", IEEE, USA, To be sent.

This thesis consists of three parts:

Part I (Chapter 2) derives a new formula for bed height calculation based on the first principles (physical laws) of a cold flow circulating fluidized bed.

Part II (Chapter3-5) deals with the system identification of a cold flow circulating fluidized bed describing it as a nonlinear device, a linear time invariant (LTI) device and a linear time varying (LTV) system. To incorporate the wide range of CFCFB's operating condition considered in Chapter 3, a nonlinear model is chosen, while the LTI model in Chapter 4 is developed in a single operating regime of the riser achieved with a cork particulate material. The move air flow split between the standpipe and the L-valve, and additional aerations routes within the standpipe need to be determined online during operation and hence, a LTV model is opted as a good choice for the same machine in Chapter 5.

Part III (Chapter 6) discusses the adaptive PID control of the CFCFB operating near the fast fluidized regime with glass beads material.

There is a similarity between Chapter 3 and Chapter 4 consisting of a prediction-error-method (PEM) to minimize a cost function between measured and predicted output in order to identify model parameters; however, the difference lies in the linear and nonlinear description of the system model. The recursive prediction error method (RPEM) used in Chapter 6 depends on the material presented in Chapter 5. Part I can be read independently from Parts II and III. Conclusions and recommendations for further research can be found in Chapter 7.

Notations are presented at the end of the thesis to avoid confusion that may arise by using the same symbols for different parameters. This is especially the case with system identification chapters using the same notations for input and output of the system to comply with the conventions used in Control community. 


\section{Chapter 2}

\section{Bed Height Calculation}

\subsection{Motivation}

Bed height is one of the factors that is important for the successful operation of a circulating fluidized bed (CFB) unit. It is useful for assessing system performance and to prevent poor temperature control, poor gas solids separation performance, flow reversal up the standpipe, etc. The standpipe bed level can provide the guidance on the needed action to bring the CFB under control when a drop in circulation rate is indicated. When taken in conjunction with the fluidization regime in the riser, the relative gas-solids velocity in the standpipe, and the distribution of standpipe aeration, the standpipe bed level can signal either a loss in solids capture efficiency, excessive attrition, flooding or defluidization in the standpipe or any other number of potential solids flow problems. Hence, it is desirable to obtain a formula that could be used to calculate the bed height and to keep the height of solids in the standpipe optimally at a desired level during operation entailed by some control means. A new formula has been derived based on a 2region model concept for bed height calculation.

\section{$2.2 \quad$ Packed Beds and Fluidized Beds}

A fixed or packed bed refers to a bed of stationary particles residing on a perforated grid, through which a gas flows. In moving packed beds, the solids move with respect to the walls of the column, like those in the return leg of a CFB. In either case, the particles do not move relative to each other. When the gas flows through a packed bed of solids it exerts a drag force on the particles, causing a pressure drop across the bed.

A typical packed bed is a cylindrical column that is filled with a suitable packing material. The information on different types of packing material can be found in Perry and Green [30]. From a fluid mechanical perspective, the most important issue is this pressure drop required for the liquid or the gas to flow through the column at a specified flow rate. To calculate this quantity, a friction factor correlation attributed to Ergun is normally used, Geldart [31].

A fluidized bed is a packed bed through which a fluid flows at such a high velocity that the bed is loosened and the particle-fluid mixture behaves as though it is a fluid. Thus, when a bed of particles is fluidized, the entire bed can be transported like a fluid, if desired. Both gas and liquid can be used to fluidize a bed of particles.

\subsection{Bed Height Calculation}

Bed height calculation is carried out using the new formula based on a 2-region model. In this model, basically, there are two homogeneous regions - Lean and Dense - and the bed height $H_{s p}$ separates these two regions as shown in Fig. (2.1). Lean region is defined as the region where the bulk solid density is low and the region with high bulk solid density is said to be a dense region.

Generally, when the gas (air) is fed through the bottom of the standpipe, the net weight of the bed has to be balanced against the upward force exerted on the bed, namely the pressure drop across the bed, $\Delta P$ multiplied by a cross-sectional area of the bed $A$. In doing this balance, the small frictional force exerted on the wall of the column by the flowing fluid is neglected. 
Upward force on the bed $=\triangle P A$

If the height of the bed at this point is $L$ and the void fraction is $\varepsilon$, we can write

Volume of particles $=(1-\varepsilon) A L$

If the acceleration due to gravity is $g$, the net gravitational force on the particles (net weight) is $(1-\varepsilon)\left(\rho_{p}-\rho_{g}\right) A L g$.

Balancing the two yields

$$
\Delta P=\left(\rho_{p}-\rho_{g}\right)(1-\varepsilon) L g
$$

where $\rho_{p}$ is the solids particle density in $\mathrm{Kg} / \mathrm{m}^{3}$ and $\rho_{g}$ is the gas particle density $\mathrm{Kg} / \mathrm{m}^{3}$.

The symbols appearing in Fig. (2.1) are defined as follows:

$\Delta P_{T}, L_{s p}$ : Total pressure drop and total height of the standpipe in Pascal and meter, respectively.

$\Delta P_{L}, z_{L}$ : Overall pressure drop in the lean region in Pascal and the height corresponding to lean phase pressure drop in meter.

$\Delta P_{D}, H_{s p}$ : Overall pressure drop in the dense region in Pascal and the height corresponding to dense phase pressure drop in meter.

$\Delta P, z$ : Measured pressure drop in Pascal and measured height in meter in the dense region.

$\Delta P_{h}, h$ : Portion of pressure drop in Pascal and height in meter in the dense region to be determined.

To derive a formula for calculating bed height, Eq. (2.1) has been utilized in the lean region of the stand pipe. With the present data cork data set, $\rho_{p}-\rho_{g} \approx \rho_{p}$, therefore, Eq. (2.1) reduces to

$$
\Delta P_{L}=\rho_{p}\left(1-\varepsilon_{L}\right) g z_{L}
$$

where $\varepsilon_{L}$ is the void fraction in the lean region and $\Delta P_{L}$ is the lean phase pressure drop. The bed height is calculated using a pressure balance equation in the standpipe, which is expressed as follows:

$$
\begin{aligned}
\Delta P_{T} & =\Delta P_{L}+\Delta P_{D} \\
& =\rho_{p}\left(1-\varepsilon_{L}\right) g z_{L}+\left(\Delta P+\Delta P_{h}\right)
\end{aligned}
$$

$\Delta P_{h}$ can be found from the proportional relationship between pressure drop and height as follows:

$$
\begin{aligned}
& \frac{\Delta P_{D}}{\Delta P}=\frac{H_{S P}}{z} \\
& \text { or, } \frac{\Delta P+\Delta P_{h}}{\Delta P}=\frac{z+h}{z}
\end{aligned}
$$




$$
\begin{aligned}
& \text { or }, 1+\frac{\Delta P_{h}}{\Delta P}=1+\frac{h}{z} \\
& \therefore \Delta P_{h}=\Delta P \frac{h}{z}
\end{aligned}
$$

Substituting the value of $\Delta P_{h}$ in Eq. (2.3) and manipulating, we get,

$$
h=\frac{\Delta P_{T}-\Delta P-\rho_{p}\left(1-\varepsilon_{L}\right) g\left(L_{S P}-z\right)}{\frac{\Delta P}{z}-\rho_{p}\left(1-\varepsilon_{L}\right) g}
$$

Hence, the bed height, $H_{S P}=z+h$

The pressure drop $\Delta P$ in Eq. (2.3) is considered at $4.6 \mathrm{~m}$ above the bottom of the standpipe. In the CFCFB, the bed height is usually kept above $6 \mathrm{~m}$. With respect to the test operations at NETL, the CFB cold flow unit is typically operated with the bed above the spiral solids flow meter located about $4 \mathrm{~m}$ above the loop seal. Additional solids are being added when the bed level goes below $6 \mathrm{~m}$ height. A relatively safe high inventory of solids is chosen to avoid problems with the measurement device that will read inaccurate values if the spiral vane becomes exposed.

The lower section of the bed is denser and therefore, it results in higher pressure drop per unit height of the bed relative to the upper section, which is leaner. For a given amount of inventory, the solids are distributed between the bed and the return leg in such a way that the pressure drops across two legs of the loop balance each other out. Thus, we see a pressure profile being closed around the CFB loop.

The pressure drop across the non-mechanical valve controlling the solids flow is proportional to its flow rate. Therefore, when the solids flow rate is increased by increasing the aeration air at the base of the standpipe, the pressure drop at the bottom increases, and the pressure drop across the moving bed in the return leg increases from its previous value. The pressure drop across the riser as well as the cyclone also changes.

The point where the change takes place and separates the lean and dense regions of the standpipe as illustrated in a bottom right corner of Fig. (2.1), is a function of the solids circulation rate and solids inventory. Because the experiment is really a closed system with respect to the bed material (that is to say those solids do not continuously enter or leave the system), then the amount of material in the riser and standpipe during operation is usually present in the standpipe before we begin circulation. The maximum amount of material we can easily accommodate in the system "at rest" has the standpipe full and solids sitting in the primary cyclone. After we begin circulating solids, the total amount of material splits between the riser and the standpipe such that the standpipe bed height is between 6 and 11 meters. The variation in the standpipe bed height depends on the circulation rate and the gas flow through the riser. 


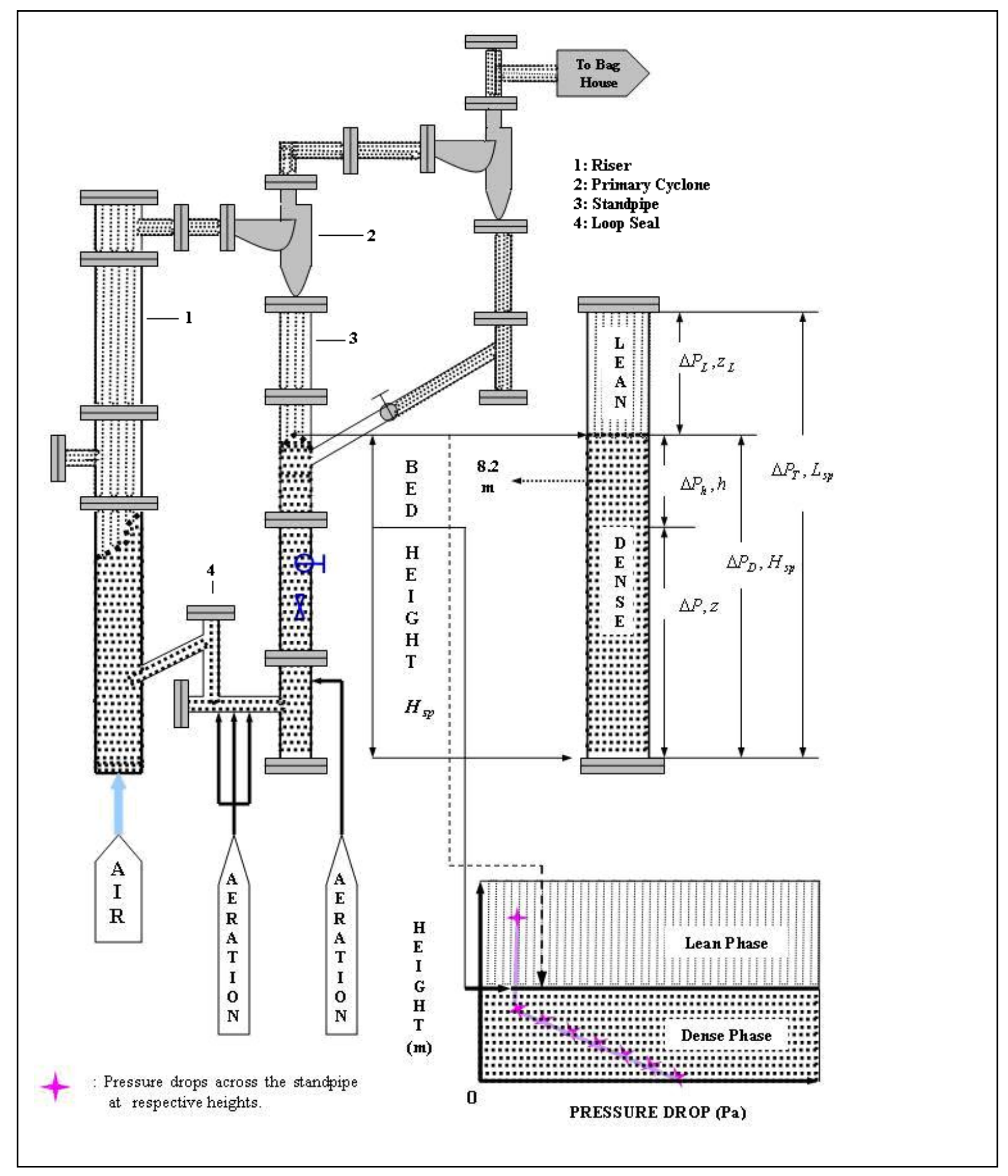

Figure (2.1): Schematic of a cold flow circulating fluidized bed. Also shown are the two region model of the standpipe and a plot of pressure profile in the standpipe.

Finally, there are experiments performed where we need to watch the standpipe bed height as a function of time as in the present case. These experiments are conducted either during continuous operation or 
during the transient when the solids circulation is halted and the riser clears its inventory back into the standpipe. In either case, we need to watch the bed height and the 6-11 meter height is convenient.

\section{$2.4 \quad \varepsilon_{L}$ Values}

As can be seen from Eqs. (2.3) - (2.4), the bed height formula involves the lean phase void fraction whose measurements are not available from the cold unit at NETL. In Rupen et. al. [18], it was chosen by a trial and error and $0.98<\varepsilon_{L}<1$ was found to be reasonable for the present sets of data.

\subsection{Results and Discussions}

At present, there is no particular device installed in the given set up to measure bed height in the standpipe. A portion of the standpipe wall is made of clear acrylic for visual observation so that the bed height can be observed and recorded. The bed height is recorded once every 15 seconds and the sampling rate is converted to $1 \mathrm{~Hz}$ using shape preserving interpolant under MATLAB environment.

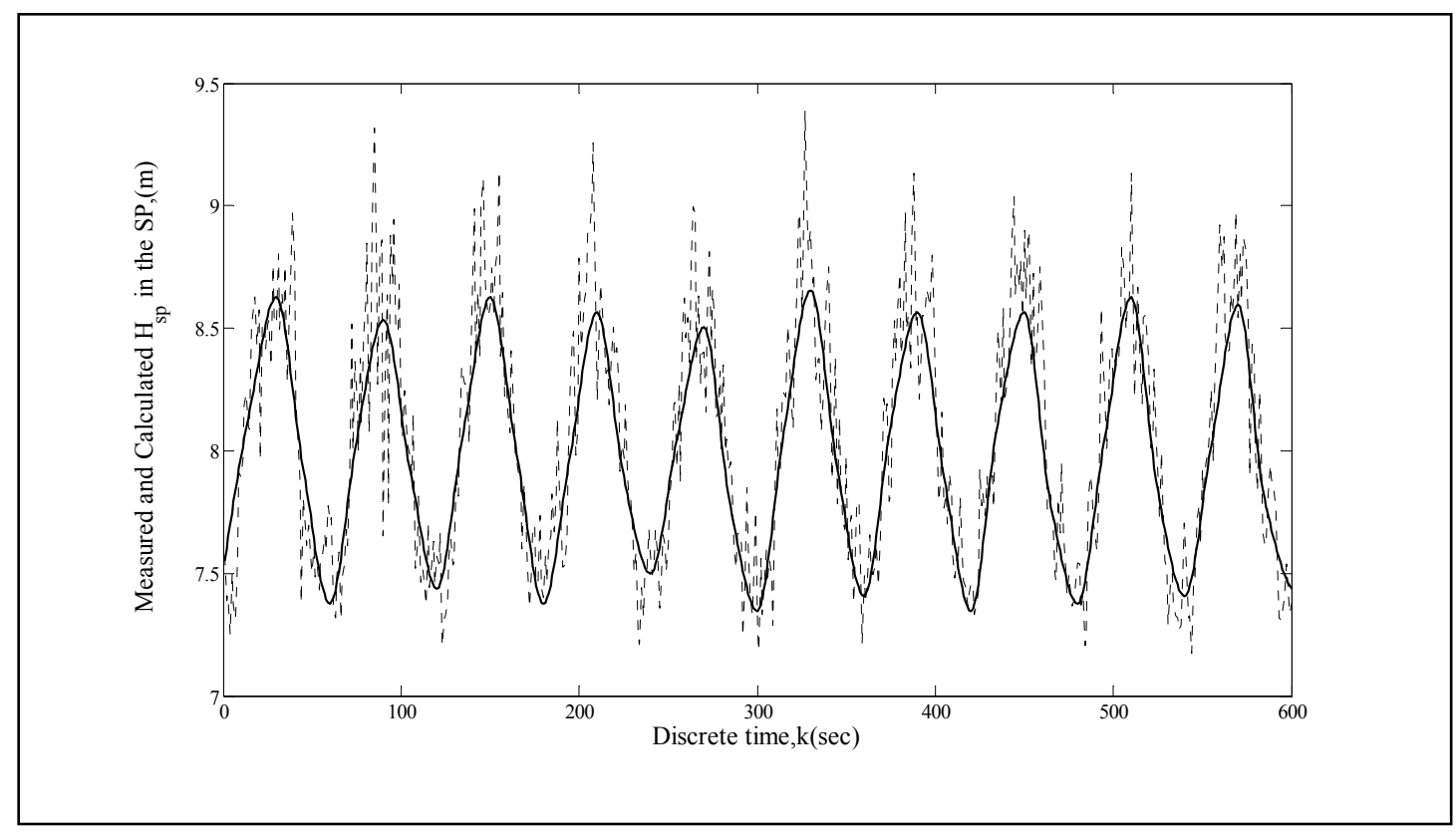

Figure (2.2): Comparison of measured and calculated bed height around $8.2 \mathrm{~m}$.

It seems that the variations in the bed height data are dominated by observation errors only rather than the process noise similar to those acting upon solids circulation rate measured by a spiral vane or as in the case of pressure tap measurements and hence, the bed height observations appear smooth as shown in Figs. (2.2) and (2.3).

Both the measurements and simulation results for bed height are shown in Figs. (2.2) and (2.3) for the case when the bed height is above $8.2 \mathrm{~m}$ and around $8.2 \mathrm{~m}$. In these cases, a solid black line and a dotted black line represent measured and simulated values, respectively. The simulated results involve the measured values of pressure drops and hence, contain the noise associated with these measurements. 


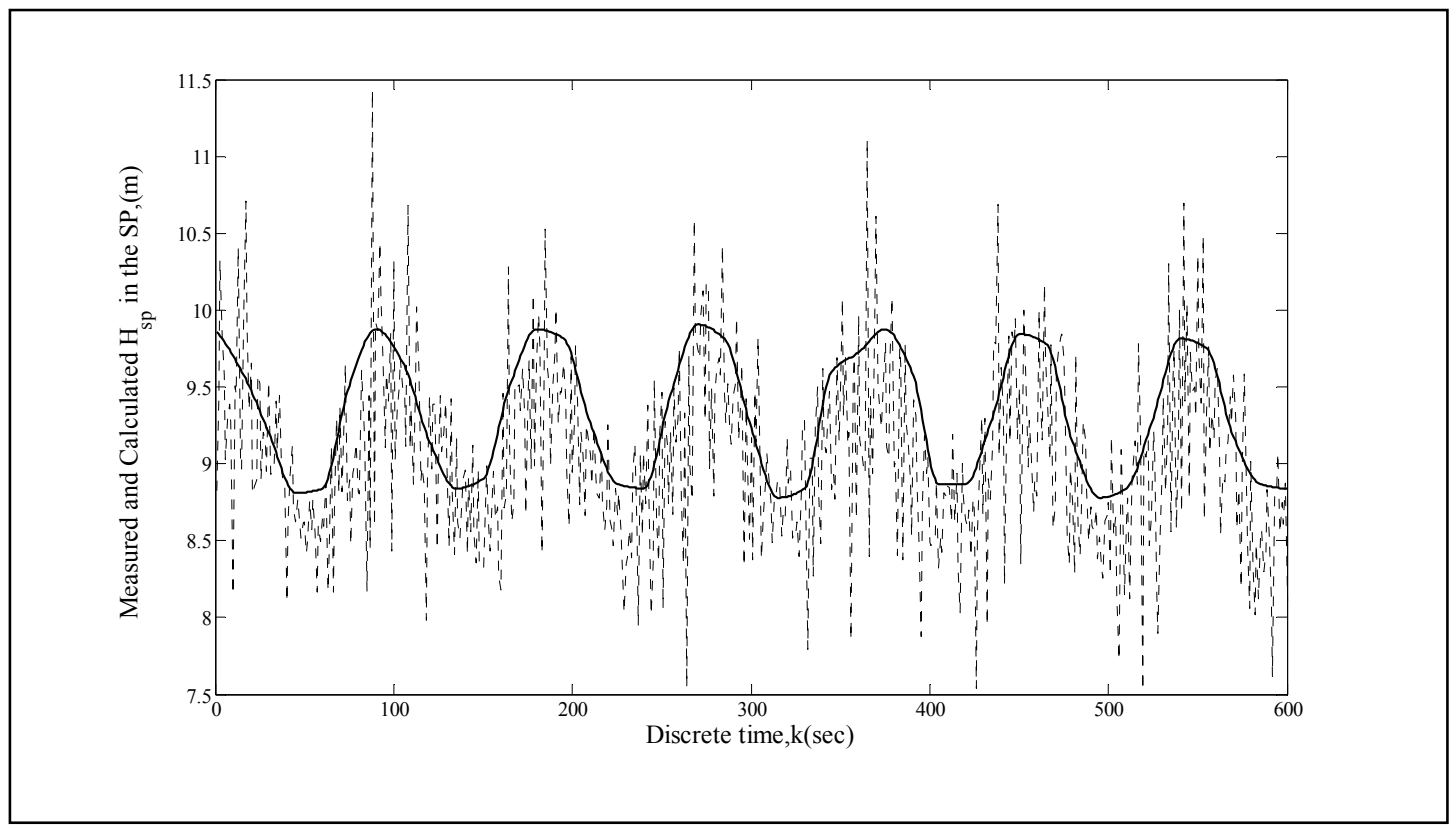

Figure (2.3): Comparison of measured and calculated bed height greater than $8.2 \mathrm{~m}$.

In summary, a new formula has been derived for bed height calculation based on a 2-region model concept. The $\varepsilon_{L}$ value required for the calculation is chosen between 0.98 and 1 for two different experiments where the bed height is $8.2 \mathrm{~m}$ and around $8.2 \mathrm{~m}$. The rational behind maintaining bed height in the standpipe of a CFCFB during experiments at a cold model present in NETL is carefully explained. Simulation results are compared with observed values of the bed height in the experimental plots. 


\section{Chapter 3}

\section{Multiple Model Identification of a Cold Flow Circulating Fluidized Bed}

\subsection{Motivation}

Multiple model identification is a novel method that allows the determination of local linear state space models from input and output data to approximate a nonlinear dynamic system. CFBs may exhibit different behaviors when operating in different flow regimes: bubbling, slugging, turbulent, fast, and transport and because each flow regime tends to be explained by its own model, intuition would indicate that multiple model identification would seem a worthwhile method to predict the behavior of a CFB across its entire operating range. Note though that the multiple model identification method does not use or find models for the different flow regimes. Rather it assumes that the behavior of a nonlinear dynamic system such as a CFCFB is explained as the weighted behavior of several unknown linear states, which are called local models. Unless a transition between states is occurring, one of the linear states is dominant and its weight is much greater than the others. The approach is to use measurements of the riser aeration, move air flow, the total riser pressure drop, and the spiral vane to first train the model and then use the model to predict SCR from the riser aeration, move air flow and total riser pressure drop.

\subsection{Multiple Model Identification: Basic Theory}

Let us assume that the solids circulation rate, $y(k)$, at discrete time $k$ depends on the move air flow, riser aeration and total riser pressure drop and the solids circulation rate at the previous time instant $k-1$, i.e.,

$y_{k}=h\left(y_{k-1}, u_{k-1}\right) \Rightarrow y_{k+1}=h\left(y_{k}, u_{k}\right)$

where $h$ is a continuous function with certain smoothness properties and $u_{k}=[\operatorname{Move} \operatorname{Air}$ Flow $(k)$, Riser Aeration $(k)$, Total Riser Pressure Drop $(k)]$.

Let us now decompose the operating range of the given system (3.1), into a certain number of subsets in which a linearized model is believed to be a reasonable approximation of the given system. These subsets are termed as the operating regimes of the systems. The system (3.1) is then divided into $s$ distinct operating regimes and each regime is combined to form an entire regime of the CFCFB through a weighting function $p_{i}\left(\varphi_{k}\right) \in \mathfrak{R}$ as follows:

$y_{k+1}=\sum_{i=1}^{s} p_{i}\left(\phi_{k}\right) h\left(y_{k}, u_{k}\right)$

where $p_{i}\left(\varphi_{k}\right)$ has a local support that satisfy

$0 \leq p_{i}\left(\phi_{k}\right) \leq 1, \quad \sum_{i=1}^{s} p_{i}\left(\phi_{k}\right)=1, \quad i=1,2,3, \ldots, s$ 
and $\phi_{k}=\psi\left(y_{k}, u_{k}\right)$ is the scheduling parameter that schedules the system from one operating regime to the other. The weighting function $p_{i}\left(\varphi_{k}\right)$ is close to one in the $i^{\text {th }}$ operating regime while the other weighting functions are zero. In the transitions between the operating regimes, the weights smoothly vary from approximately zero to approximately one and vice versa.

The function $h$ is linearized at an operating point $\left(y_{e}^{i}, u_{e}^{i}\right)$ in every operating regime with the Taylor series expansion as:

$h\left(y_{k}, u_{k}\right) \approx h\left(y_{e}^{i}, u_{e}^{i}\right)+\left(y_{k}-y_{e}^{i}\right) \frac{\partial h}{\partial y_{k}^{T}}\left(y_{e}^{i}, u_{e}^{i}\right)+\left(u_{k}-u_{e}^{i}\right) \frac{\partial h}{\partial u_{k}^{T}}\left(y_{e}^{i}, u_{e}^{i}\right)$

neglecting higher order terms. At each operating point, the function $h$ becomes

$$
h\left(y_{k}, u_{k}\right)=M_{i} y_{k}+N_{i} u_{k}+\left[h\left(y_{e}^{i}, u_{e}^{i}\right)-M_{i} y_{e}^{i}-N_{i} u_{e}^{i}\right]=M_{i} y_{k}+N_{i} u_{k}+Q_{i}
$$

with

$$
\begin{aligned}
M_{i} & :=\frac{\partial h}{\partial y_{k}^{T}}\left(y_{e}^{i}, u_{e}^{i}\right) \\
N_{i} & :=\frac{\partial h}{\partial u_{k}^{T}}\left(y_{e}^{i}, u_{e}^{i}\right) \\
Q_{i} & :=h\left(y_{e}^{i}, u_{e}^{i}\right)-M_{i} y_{e}^{i}-N_{i} u_{e}^{i},
\end{aligned}
$$

$Q_{i}$ is also termed as the offset in the dynamic linearization of a given system (3.1), Verdult et. al. [24], Johansen et. al. [32].

Substituting Eq. (3.5) into Eq. (3.2), we get,

$$
y_{k+1}=\sum_{i=1}^{s} p_{i}\left(\phi_{k}\right)\left(M_{i} y_{k}+N_{i} u_{k}+Q_{i}\right)
$$

Eq. (3.6) can be rewritten as

$$
y_{k}=\left(q I-\sum_{i=1}^{s} p_{i}\left(\phi_{k}\right) M_{i}\right)^{-1} \sum_{i=1}^{s} p_{i}\left(\phi_{k}\right)\left(N_{i} u_{k}+Q_{i}\right)
$$

where $q$ is the forward time shift operator defined as $q z_{k}=z_{k+1}$. Let us define 
$C\left(q I-\sum_{i=1}^{s} p_{i}\left(\phi_{k}\right) A_{i}\right)^{-1} \sum_{i=1}^{s} p_{i}\left(\phi_{k}\right)\left(B_{i} u_{k}+O_{i}\right)=\left(q I-\sum_{i=1}^{s} p_{i}\left(\phi_{k}\right) M_{i}\right)^{-1} \sum_{i=1}^{s} p_{i}\left(\phi_{k}\right)\left(N_{i} u_{k}+Q_{i}\right)$

or, $C x_{k}=\left(q I-\sum_{i=1}^{s} p_{i}\left(\phi_{k}\right) M_{i}\right)^{-1} \sum_{i=1}^{s} p_{i}\left(\phi_{k}\right)\left(N_{i} u_{k}+Q_{i}\right)$

where, $x_{k}=\left(q I-\sum_{i=1}^{s} p_{i}\left(\phi_{k}\right) A_{i}\right)^{-1} \sum_{i=1}^{s} p_{i}\left(\phi_{k}\right)\left(B_{i} u_{k}+O_{i}\right)=$ State of the CFCFB at time instant $k$

Using the definition of the $q$ operator and manipulating the last term of Eq. (3.7), we get,

$x_{k+1}=\sum_{i=1}^{s} p_{i}\left(\phi_{k}\right)\left(A_{i} x_{k}+B_{i} u_{k}+O_{i}\right)$

Hence, the general nonlinear state space model that is a collection of linearized models for the CFCFB is defined as:

$x_{k+1}=\sum_{i=1}^{s} p_{i}\left(\phi_{k}\right)\left(A_{i} x_{k}+B_{i} u_{k}+O_{i}\right), \quad$ a state equation in a nonlinear difference form.

$y_{k}=C x_{k}, \quad$ an algebraic output equation.

It is clear from the above derivation that there is no particular meaning of the state $x_{k}$ that can be interpreted as any physical variable of the given system. The state $x_{k}$ is introduced as a fictitious intermediate variable to connect input to the output of the given set up.

If the contribution of unknown white noise $v_{k} \in \mathfrak{R}$ acting on the solids circulation rate measurement is considered, then the description of Eq. (3.9) becomes

$x_{k+1}=\sum_{i=1}^{s} p_{i}\left(\phi_{k}\right)\left(A_{i} x_{k}+B_{i} u_{k}+O_{i}\right)$

$y_{k}=C x_{k}+v_{k}$

Furthermore, if it is assumed that the measurement error $v_{k}$ propagates to the internal dynamics of the system through a matrix $K_{i}$ in each operating regime, we get a new system as follows:

$x_{k+1}=\sum_{i=1}^{s} p_{i}\left(\phi_{k}\right)\left(A_{i} x_{k}+B_{i} u_{k}+O_{i}+K_{i} v_{k}\right)$

$y_{k}=C x_{k}+v_{k}$

Instead of introducing a fictitious vector $x_{k}$, the given system (3.1) can be directly implemented in the input-output form as presented in Eq. (3.5). However, state space systems are more attractive for dealing with multivariable inputs and outputs as with our CFCFB case. This is because the input-output description requires more complicated structure to achieve results commensurate with those of state-space 
model, than Eq. (3.1). For example, such description may require more time lagged inputs and outputs (Eq. (3.12)) rather than single time lagged inputs and outputs as in Eq. (3.1):

$y_{k+1}=h\left(y_{k}, y_{k-1}, y_{k-2,}, \ldots, y_{k-P}, u_{k}, u_{k-1}, u_{k-2}, \ldots, u_{k-P}\right)$

On the other hand, state space systems are likely to require fewer parameters, especially for multivariable systems. One of the reasons is that the number of time-lagged inputs and outputs $P$ used in the inputoutput description (3.12) satisfies $n \leq P \leq 2 n+1$ with $n$ the order of the state space realization of this system, provided it exists, Stark et. al.[33],. Stark [34]. In addition, many nonlinear control methods require a state-space model of the system. Because of these reasons, state-space models are often preferred to input-output models. In the present case, a fictitious state vector is introduced to attain a desired result only by defining the CFCFB in the form of Eq.(3.1), that would have been otherwise obtained using a system description (3.12). This, however, leads to the loss of interpretation of $x_{k}$ which is not associated with any physical parameters of the CFCFB.

\subsection{Prediction Error Method (PEM)}

System identification is usually carried out by minimizing the difference between the output of a pre selected model and the measured output of the system to be identified. The model mismatch is quantified by a cost function that decreases when the output predicted by the model approaches the measured output. A commonly used cost function is the sum of squared errors between the predicted output and the measured output for a fixed, large number of samples. When the model is completely described by a set of parameters, system identification boils down to finding the values of the parameters that minimize this cost function. This approach is commonly known as prediction-error approach.

The one-step-ahead predictor for the CFCFB can be defined such that the cost function between the measured and predicted solids circulation rate is minimized to identify its parameters. Such a predictor is derived from Eq. (3.11) that is given below:

$$
\begin{aligned}
& \hat{x}_{k+1}=\sum_{i=1}^{s} \hat{p}_{i}\left(\phi_{k}\right)\left(\left(\hat{A}_{i}-\hat{K}_{i} \hat{C}\right) \hat{x}_{k}+\hat{B}_{i} u_{k}+\hat{O}_{i}+\hat{K}_{i} y_{k}\right) \\
& \hat{y}_{k}=\hat{C} \hat{x}_{k}
\end{aligned}
$$

where $\hat{x}_{k} \in \mathfrak{R}^{n}, \hat{p}_{i}\left(\phi_{k}\right) \in \mathfrak{R}$, and $\hat{y}_{k} \in \mathfrak{R}^{\ell}$ are the estimated values. $\hat{A}_{i}, \hat{B}_{i}, \hat{O}_{i}, \hat{K}_{i}$ and $\hat{C}$ are the system matrices to be determined from the experimental data.

\subsection{Estimating the Predictor Parameters}

The identification algorithms based on a multiple model approach that is used to obtain the predictor (3.13) can be seen from the following block diagram (Fig. (3.1)). Here, we have to present measured inputs and output to the algorithm to obtain the predictor (3.13). Riser aeration, move air flow, and total riser pressure drop are the inputs, $u_{k}$, to the one-step-ahead predictor (3.13). The solids circulation rate is the measured output $y_{k}$. The prediction of the solids circulation rate is $\hat{y}_{k}$. 


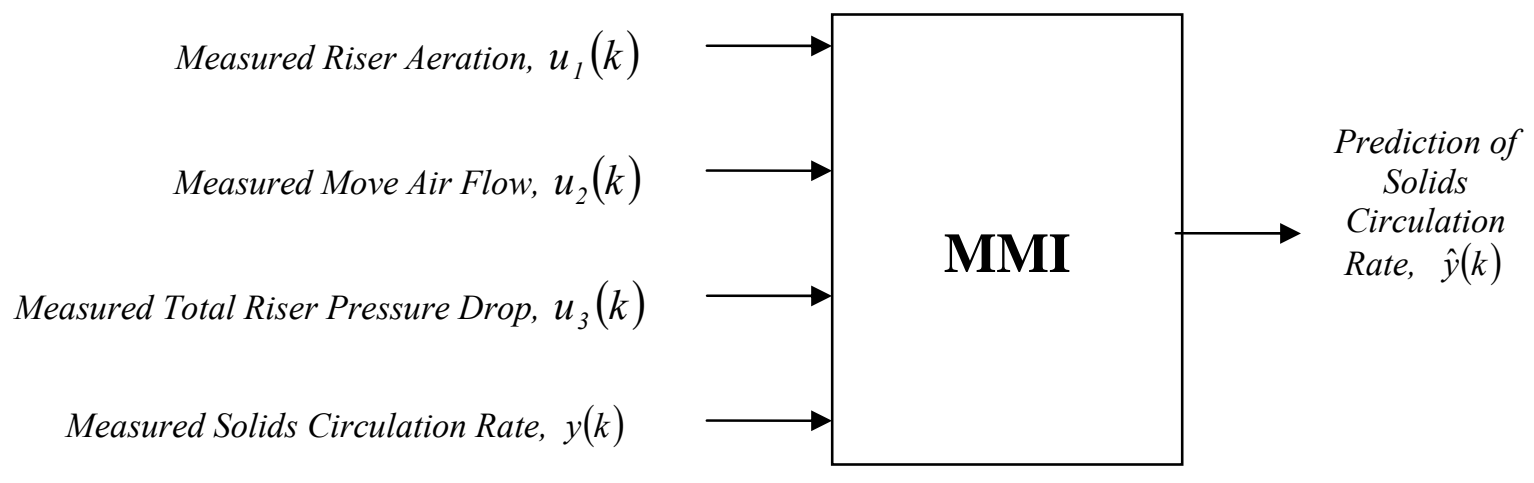

Figure (3.1): Block diagram for prediction of solids circulation rate using MMI.

In the output-error identification method that corresponds to the system description with white measurement noise only at the output (Eq. (3.10)) and with the prediction-error equals the difference between the measured output and the simulated output of the model, it is necessary to supply both measured inputs and output to the algorithm to determine predictor parameters during identification. During validation phase, only inputs need to be supplied to predict the future values of solids circulation rate. In the prediction-error method that considers process noise and measurement noise acting on the system as in Eq. (3.14), both measured inputs and output should be provided during identification and validation stages as it is not possible to estimate a process noise a priori acting on the system. In this work, the output-error identification method was attempted to predict the solids circulation rate in the beginning but the results obtained were not improved as those obtained from the prediction-error method.

As with many prediction error methods, the predictor output depends nonlinearly on the parameters that describe the predictor and hence, the minimization of the prediction-error cost function becomes a nonlinear, nonconvex problem that can have several local minima. It is common practice to use a gradient search method known as the Levenberg-Marquardt algorithm to solve this optimization problem. In this case, the initial starting point determines in which local minimum the algorithm will end up. Therefore, it is of paramount importance to have a good initial estimate of the given system available when using prediction-error methods.

Subspace identification methods like N4SID are notable exception to this approach. Some of them aim at recovering the column space of the extended observability matrix and use the shift-invariant structure of this matrix to estimate the matrices $A$ and $C$ and use these approximate matrices in a second step to estimate the matrix $B$ and the initial state $x(0)$. They do not require a particular parameterization of the system; this makes them numerically attractive and especially suitable for multivariable linear time invariant systems of the following form.

$$
\begin{aligned}
& x_{k+1}=A x_{k}+B u_{k}+K e_{k} \\
& y_{k}=C x_{k}+e_{k}
\end{aligned}
$$

where $e_{k}$ is called the "innovation" that represents that part of the output $y_{k}$ that cannot be predicted from the past data, Ljung [25]. 
The block diagram shown in Fig. (3.2) indicates the prediction of solids circulation rate using N4SID algorithm. In order to identify the parameters of a linear system in the presence of measurement and process noise using N4SID, both inputs and outputs need to be provided during estimation as well as validation phase.

Subspace methods can be used to generate an initial starting point for iterative prediction-error methods. This makes it less likely that the prediction-error method ends up in a local minimum that corresponds to a bad model performance. This combination of two methods is a powerful tool to tackle multiple model identification problems.

All the local models are initialized using the system matrices estimated by N4SID as in Eq. (3.14). This leads to better results than that predicted by a linear model, Sjöberg et. al. [35] as would be shown through the plots of the experimental results. All $O_{i}^{\prime} s$ are initialized at the arbitrary equilibrium point $(x(0), u(0))$.

Moreover, the initial value of the state $x(0)$ is required. It is also obtained from the N4SID routine. In this routine, $A$ and $C$ are estimated from the extended observability matrix that is formed from the input-output data, and the matrix $B$ and the initial state $x(0)$, if required, can be determined from the following predictor, [25]:

$\hat{y}(k \mid B, x(0))=\hat{C}(q I-\hat{A})^{-1} x(0) \delta_{k}+\hat{C}(q I-\hat{A})^{-1} B u_{k}$

using least squares like method:

$\underset{B, x(0)}{\arg \min } \frac{1}{N} \sum_{k=1}^{N}\left\|y_{k}-\hat{C}(q I-\hat{A})^{-1} B u_{k}-\hat{C}(q I-\hat{A})^{-1} x(0) \delta_{k}\right\|_{2}^{2}$

Here, $N$ is the total number of data samples, $\delta_{k}$ is the unit pulse at time 0 and \|\|$_{2}$ is the 2-Euclidean norm that is a 'metric' or measure of distance in Euclidean space.. The matrix $\mathrm{K}$ in Eq. (3.14) is determined from the Kalman filter algorithm once the process and measurement noise are estimated from the state and measurement equations, [25].

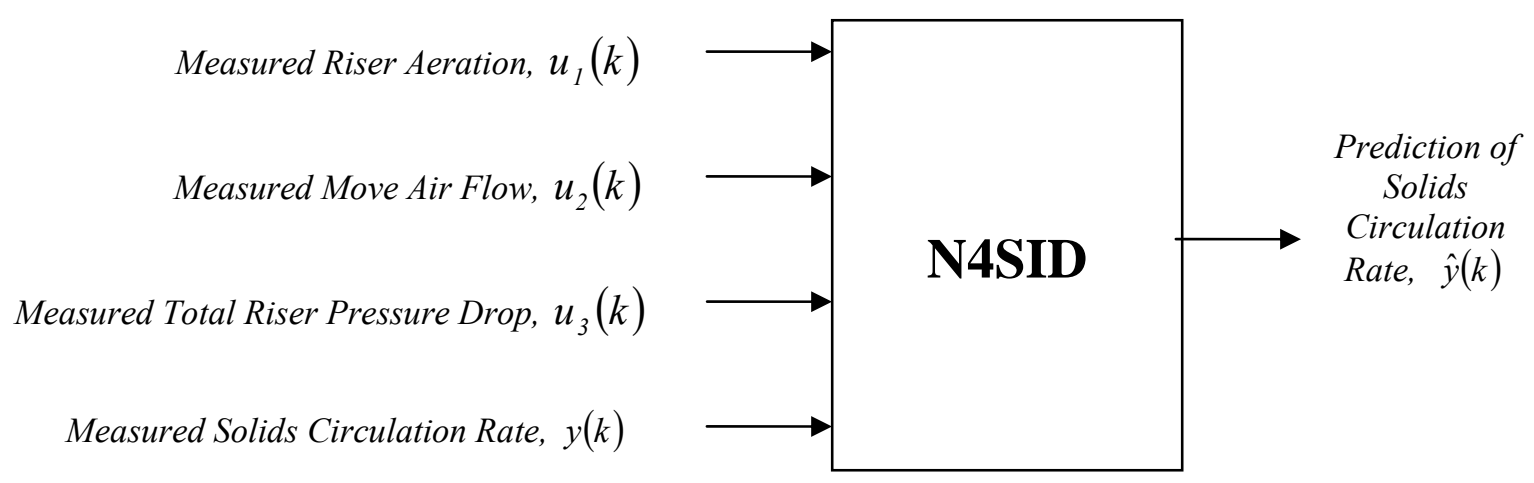

Figure (3.2): Block diagram for prediction of solids circulation rate using N4SID. 
The other requirement for using these algorithms is the desired order $n$, the number of state variables, of the given system. In N4SID routine, the singular values of the Hankel matrices of the impulse response of the given system for different orders are calculated if orders are entered as a row vector as in order $=$ [1:10]. The idea is to choose an order such that the singular values for higher orders are comparatively small. If order = 'best', a model of "best" (default choice) order is computed. With the current data set, the best model order is 3 and third order state space models are used for the prediction of solids circulation rate.

The scheduling of local models is carried out using inputs (the scheduling vector $\phi_{k}$ is taken as $u_{k}$ ) to avoid the instability problems during training that arise due to a complicated dependence of the gradient at time instant $\mathrm{k}$ with respect to the predictor parameters over the entire past of the state sequence. The weighting function $p_{i}\left(u_{k}\right)$ is chosen as a normalization of radial basis function $\left(r_{i}\left(u_{k}, c_{i}, w_{i}\right)=\exp \left(-\frac{\left(u_{k}-c_{i}\right)^{T}\left(u_{k}-c_{i}\right)}{w_{i}^{2}}\right)\right)$, where $c_{i}$ is the called the center and $w_{i}$ is called the width of the $i^{t h}$ radial basis function. To avoid subtle side effects such as reactivation of the basis functions, loss of their uniform shape and shift in minima that may arise during training with this normalization, Murray-Smith and Johansen [36], the uniform widths are used between the radial basis functions. That is, the initial widths are taken as

$$
\begin{aligned}
& w_{1}=\left\|c_{1}-c_{2}\right\|_{2}, \\
& w_{j}=\frac{1}{2}\left[\left\|c_{j}-c_{j-1}\right\|_{2}+\left\|c_{j}-c_{j+1}\right\|_{2}\right] \quad j=2,3, \ldots, s-1, \\
& w_{s}=\left\|c_{s}-c_{s-1}\right\|_{2}
\end{aligned}
$$

where the initial centers $c_{i}^{\prime} s$ are determined from fuzzy C-means clustering algorithm.

The regularized cost function is minimized using the Levenberg-Marquardt algorithm, [24], to determine the matrices $A_{i}, B_{i}, O_{i}, K_{i}$ and $C$, which describe the local models, and $c_{i}$ and $w_{i}$, which characterize the radial basis functions, from $u_{k}$ and $y_{k}$. The cost function is regularized to avoid the problem of it becoming ill conditioned in the sense that the Hessian may become an ill conditioned matrix during the training process.

A full parameterization of the system matrices is used and the gradient with respect to the predictor parameters is projected along the direction that changes the cost function while using the update rule. This is required because of the non-uniqueness of the state space representation for the predictor model defined in Eq. (3.13) that may not change the cost function at each iteration, [24]. This non-uniqueness of the state space representation is due to the similarity transformation $\widetilde{x}_{k}=T^{-1} x_{k}$, where $T \in \mathfrak{R}^{n x n}$ stands for any invertible transformation matrix, that describes the same input output behavior of the given system. The above solution can be interpreted as letting the algorithm decide the parameterization that is used at each iteration.

For training and validation of the predictors (3.13) and (3.14) (predictor for (3.14) can be similarly defined as in (3.13)), both inputs and output are normalized to [0,1] range with the following equations: 


$$
\begin{aligned}
u_{\text {normalized }} & =\frac{u-\min (u)}{\max (u)-\min (u)}, \\
y_{\text {normalized }} & =\frac{y-\min (y)}{\max (y)-\min (y)}
\end{aligned}
$$

This is done to avoid the dominance of one variable over the other while training the predictor, that is, to minimize the influence of one component with very large magnitudes as opposed to other data components that are small in magnitude.

Finally, the percentage of output variation that is explained by both of these prediction models derived from these algorithms are computed as

$$
\text { Modelfit }=100 *\left(1-\frac{\|\hat{y}-y\|_{2}}{\|y-\operatorname{mean}(y)\|_{2}}\right)
$$

where $\hat{y}$ is the estimated solids circulation rate and $y$ is the measured solids circulation rate.

When the model fits are compared, there is a significant increase in the model fit using the one-step-ahead predictor obtained by the multiple model identification technique as compared with the results obtained from N4SID as is shown in the next section.

\subsection{Results and Discussions}

Data used throughout this research were collected from the CFCFB at NETL. The training data set comprises glass beads as a bed material whose characteristics are given in Table (3.1). This data set was recorded at the test facility in September, 2006. The linear and nonlinear predictor models are validated on glass beads and cork data sets taken in September 2006, October 2006 and December 2001 respectively under different operating conditions.

\begin{tabular}{|c|c|c|}
\hline \multicolumn{2}{|c|}{ Characteristics of Glass Beads } \\
\hline Solids particle density $\left(\mathrm{Kg} / \mathrm{m}^{3}\right)$ & $\rho_{p}$ & 2488 \\
\hline Bulk density fluffed $\left(\mathrm{Kg} / \mathrm{m}^{3}\right)$ & $\rho_{b f}$ & 1457 \\
\hline Bulk density packed $\left(\mathrm{Kg} / \mathrm{m}^{3}\right)$ & $\rho_{b p}$ & 1564 \\
\hline Voidage at packed bed & $\varepsilon_{p b}$ & 0.413 \\
\hline Voidage at fluffed bed & $\varepsilon_{f b}$ & 0.37 \\
\hline Particle diameter $(\mu \mathrm{m})$ & $d_{p}$ & 180 \\
\hline Minimum Fluidization velocity $(\mathrm{m} / \mathrm{s})$ & $U_{m f}$ & 0.03 \\
\hline
\end{tabular}

Table (3.1): Characteristics of glass beads used for obtaining identification data obtained in September, 2006. 
To generate the identification data, the white noise experiment was performed in which the standpipe aeration was varied randomly between 0 and $0.14 \mathrm{~m} / \mathrm{sec}$. About $75 \%$ of the total move air flow was introduced at a point $0.4 \mathrm{~m}$ above the non-mechanical valve. The remainder of the flow was distributed uniformly along the length of the standpipe. Similarly, the riser aeration was varied randomly between 4.3 $\mathrm{m} / \mathrm{sec}$ and $10.8 \mathrm{~m} / \mathrm{sec}$. The subsequent range of the pressure drop developed across the riser between the side port, $0.23 \mathrm{~m}$ diameter and $0.27 \mathrm{~m}$ above the gas distributor, through which the solids enter the riser and the solids exit port $0.2 \mathrm{~m}$ at $90^{\circ}$ about $1.2 \mathrm{~m}$ below the top of the riser at a point $15.45 \mathrm{~m}$ above the solids entry location (centerline to centerline, Fig. (1.1)) is 0-72,000 Pascals.

The parameter values for the one-step-ahead predictors generated by the MMI and N4SID algorithms after training on the identification data are enlisted below (rounded off to 4 decimal places):

$$
\begin{aligned}
& \text { MMI: } \quad A_{1}=\left[\begin{array}{ccc}
0.3836 & -0.5694 & 0.3850 \\
-0.3945 & 0.6048 & 0.4517 \\
-0.0444 & -0.0684 & 0.7269
\end{array}\right], A_{2}=\left[\begin{array}{ccc}
0.3869 & -0.5701 & 0.3855 \\
-0.3957 & 0.6067 & 0.4159 \\
-0.0456 & -0.0670 & 0.7293
\end{array}\right] \text {, } \\
& B_{1}=\left[\begin{array}{ccc}
-0.2173 & -0.0152 & 0.1079 \\
-0.1703 & -0.0114 & 0.0704 \\
0.0264 & 0.0023 & 0.0132
\end{array}\right], B_{2}=\left[\begin{array}{ccc}
-0.2217 & -0.0065 & 0.1078 \\
-0.1667 & -0.0139 & 0.0732 \\
0.0101 & 0.0116 & 0.0117
\end{array}\right] \text {, } \\
& O_{1}=\left[\begin{array}{c}
-0.0141 \\
-0.0140 \\
0.0069
\end{array}\right], O_{2}=\left[\begin{array}{c}
-0.0119 \\
-0.0129 \\
0.0106
\end{array}\right], K_{1}=\left[\begin{array}{l}
0.1337 \\
0.0548 \\
0.0054
\end{array}\right], K_{2}=\left[\begin{array}{c}
0.1300 \\
0.0624 \\
-0.0057
\end{array}\right], \\
& C=\left[\begin{array}{lll}
7.5371 & -1.4028 & 2.8645
\end{array}\right], c_{1}=\left[\begin{array}{llll}
0.3902 & 0.3224 & 0.4150
\end{array}\right], \\
& c_{2}=\left[\begin{array}{lll}
0.6186 & 0.7926 & 0.7595
\end{array}\right], w_{1}=0.6520 \text { and } w_{2}=0.6496 \text {. } \\
& \text { N4SID: } A=\left[\begin{array}{ccc}
0.3871 & -0.5710 & 0.3854 \\
-0.3877 & 0.6082 & 0.4145 \\
-0.0464 & -0.0691 & 0.7278
\end{array}\right], B=\left[\begin{array}{ccc}
-0.2204 & -0.0099 & 0.1083 \\
-0.1691 & -0.0124 & 0.0720 \\
0.0194 & 0.0083 & 0.0120
\end{array}\right] \text {, } \\
& K=\left[\begin{array}{c}
0.1070 \\
0.0480 \\
-0.0068
\end{array}\right] \text { and } C=\left[\begin{array}{lll}
7.5368 & -1.4034 & 2.8646
\end{array}\right] \text {. }
\end{aligned}
$$

The initial state used for the MMI is

$$
x(0)=\left[\begin{array}{c}
0.0229 \\
0.0288 \\
-0.0242
\end{array}\right]
$$

The experimental conditions chosen for the glass beads material at which move air flow is modulated at period of 90 seconds are given in Table (3.2). 


\begin{tabular}{|c|c|c|c|c|c|c|}
\hline Variable & \multicolumn{6}{|c|}{ Properties } \\
\hline Bed Material & \multicolumn{6}{|c|}{ Glass Beads ( $d_{p}=180 \mu \mathrm{m}, \rho_{p}=2488 \mathrm{Kg} / \mathrm{m}^{3}$ ) } \\
\hline Riser Aeration, $(\mathrm{m} / \mathrm{s})$ & 7.34 & 5.57 & 6.06 & 5.51 & 4.22 & 4.4 \\
\hline Move Air Flow $(\mathrm{m} / \mathrm{s})$ & 0.02 & 0.013 & 0.06 & 0.02 & 0.016 & 0.016 \\
\hline Period $(s)$ & 90 & 90 & 90 & 90 & 90 & 90 \\
\hline
\end{tabular}

Table (3.2): Experimental condition at which move air flow was modulated. The experiment was performed with glass beads material in September, 2006.

The predictor is trained with the random data set that is obtained by varying move air and riser aeration one at a time. Approximately 7000 data points are used for training the predictors. The training results are shown in Fig. (3.3). Once the predictor is trained, it is validated against the modulated data set that is not seen by the predictors. The number of models is increased from $s=2$ to $s=5$ and the corresponding model fits achieved with the predictor against the validation data set are shown in Table (3.3). The validation results with the modulated data set are shown in Fig. (3.4).

The same predictor validated against the data set recorded in October 2006, in which both riser aeration and move air flow are varied simultaneously obtains a 58.7\% model fit. In this case as well, the number of models is increased from $s=2$ to $s=5$, and there is no significant difference in model fits on the validation data set by increasing the number of models, as can be seen from Table (3.4). The validation results are shown in the Fig. (3.5).

\begin{tabular}{|c|c|c|}
\hline No. of Models in MMI & MMI Model fit & \multirow{2}{*}{ N4SID Model fit, $\boldsymbol{s}=\mathbf{I}$} \\
\hline$s=2$ & $65.60 \%$ & \multirow{2}{*}{$31.80 \%$} \\
\hline$s=3$ & $65.50 \%$ & \\
\hline$s=4$ & $65.10 \%$ & \\
\hline$s=5$ & $64.50 \%$ & \\
\hline
\end{tabular}

Table (3.3): BED MATERIAL - GLASS BEADS. Number of models in a multiple model approach and the corresponding model fits for the validation data set where move air flow was modulated. Model fit for linear time invariant system obtained with N4SID algorithm is also shown. 

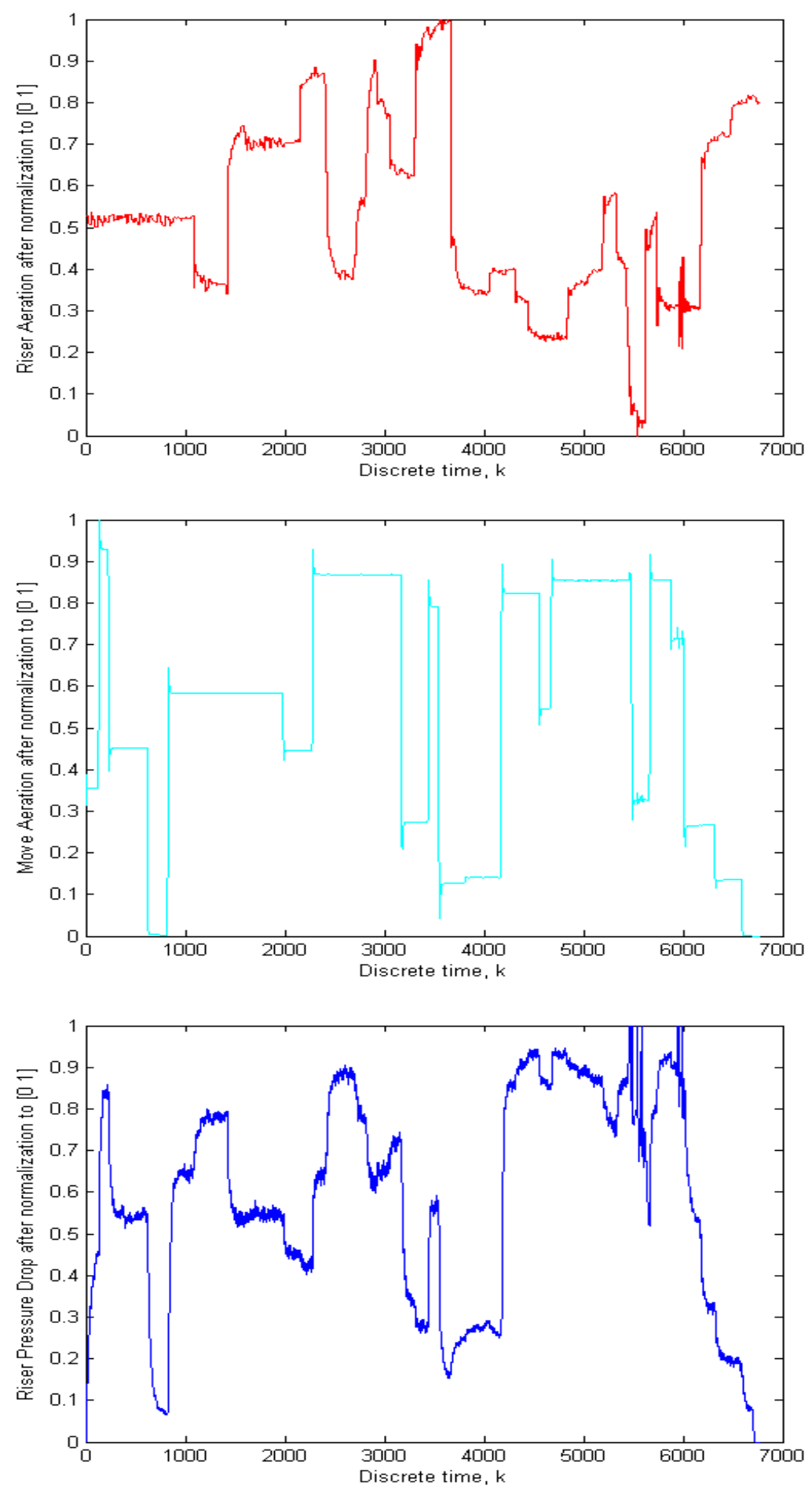

Figure $\quad 3.3 \quad$ (a): Riser aeration normalized between $[0,1]$.

Figure 3.3 (b): Move air flow normalized between $[0,1]$.
Figure 3.3 (c): Total riser pressure drop normalized between $[0,1]$.

Three inputs comprising of riser aeration, move air flow and total riser pressure drop, used to train the predictors defined in Eq. (3.13) and Eq. (3.15). 


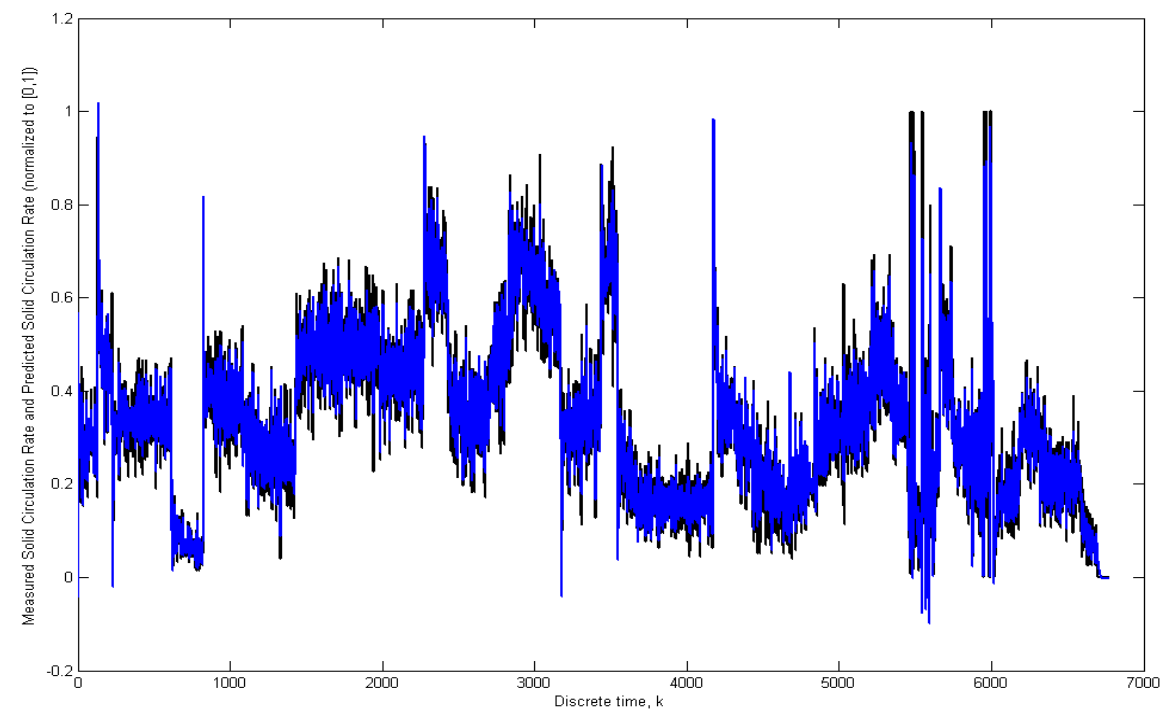

Figure 3.3 (d): Prediction of solids circulation rate using MMI, $\boldsymbol{s}=\mathbf{2}$ and $\boldsymbol{m o d e l}$ fit $=\mathbf{6 3 . 0 0} \%$. Blue line represents the predicted solids circulation rate and black line represents measured solids circulation rate. Solids circulation rate is normalized between $[0,1]$.

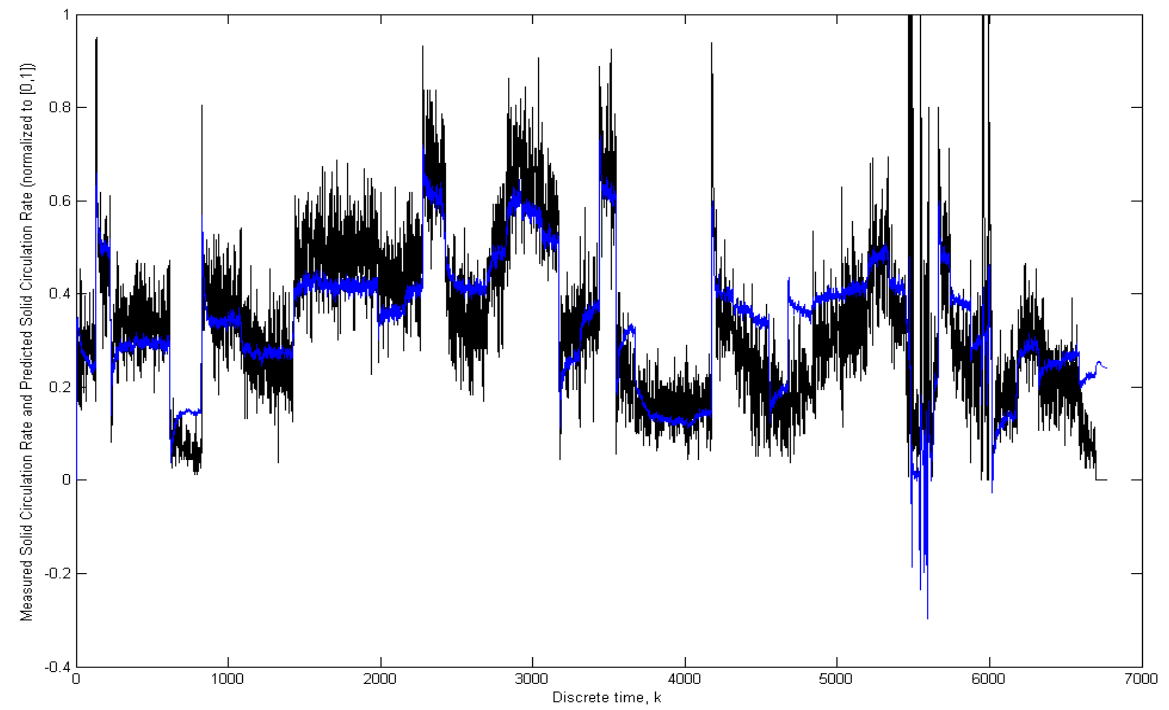

Figure 3.3 (e): Prediction of solids circulation rate using N4SID, $s=1$ and $\boldsymbol{m o d e l}$ fit $=\mathbf{3 5 . 6 7 \%}$. Blue line represents the predicted solids circulation rate and black line represents measured solids circulation rate. Solids circulation rate is normalized between $[0,1]$.

Figure (3.3): BED MATERIAL - GLASS BEADS. Training data set that comprises of riser aeration and move air varied randomly one at a time and the developed pressure drop across the riser as the inputs (taken in September 2006). Also shown is the prediction of solids circulation rate with the predictors defined in Eqs. (3.14) and (3.15). X-axis is the discrete time index. 


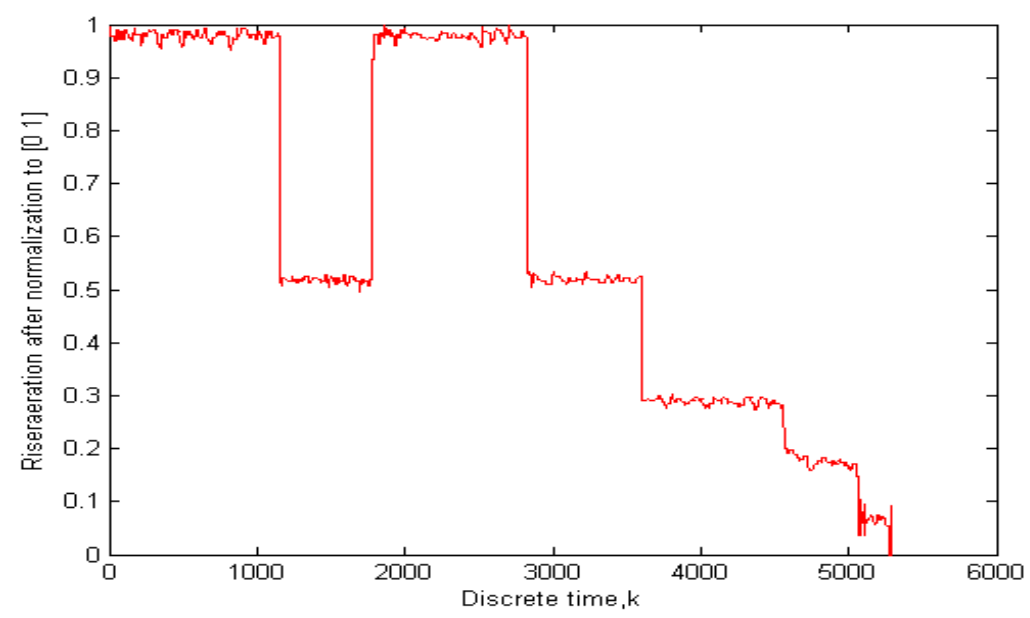

Figure $\quad 3.4 \quad$ (a): $\quad$ Riser aeration normalized between $[0,1]$.

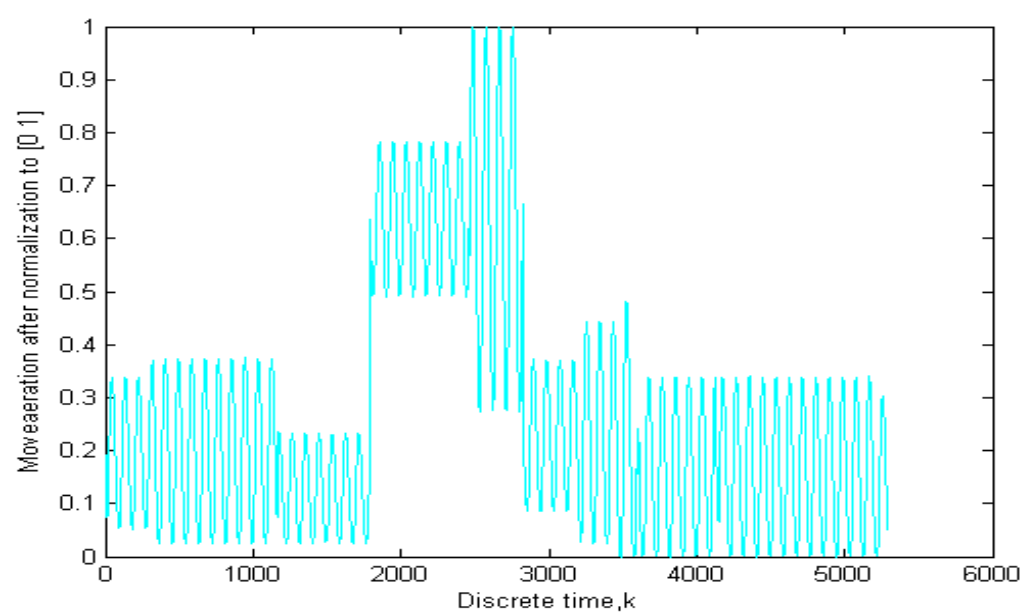

Figure 3.4 (b): Move air flow normalized between $[0,1]$.

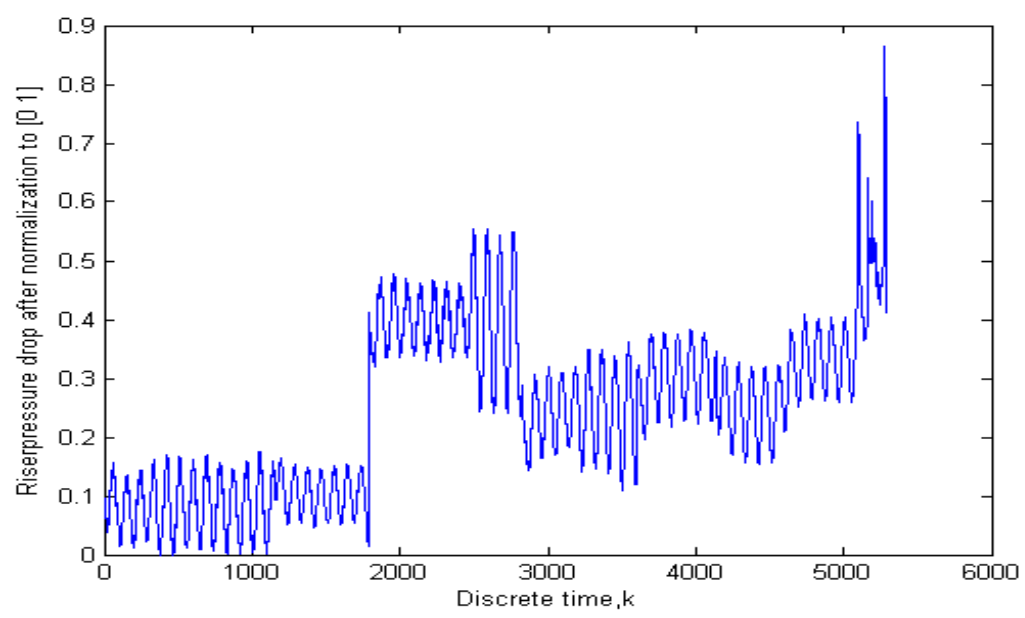

Figure 3.4 (c): Total riser pressure drop normalized between $[0,1]$.

Three inputs comprising of riser aeration, move air flow and total riser pressure drop, used to validate the predictors defined in Eq. (3.13) and Eq. (3.15). 


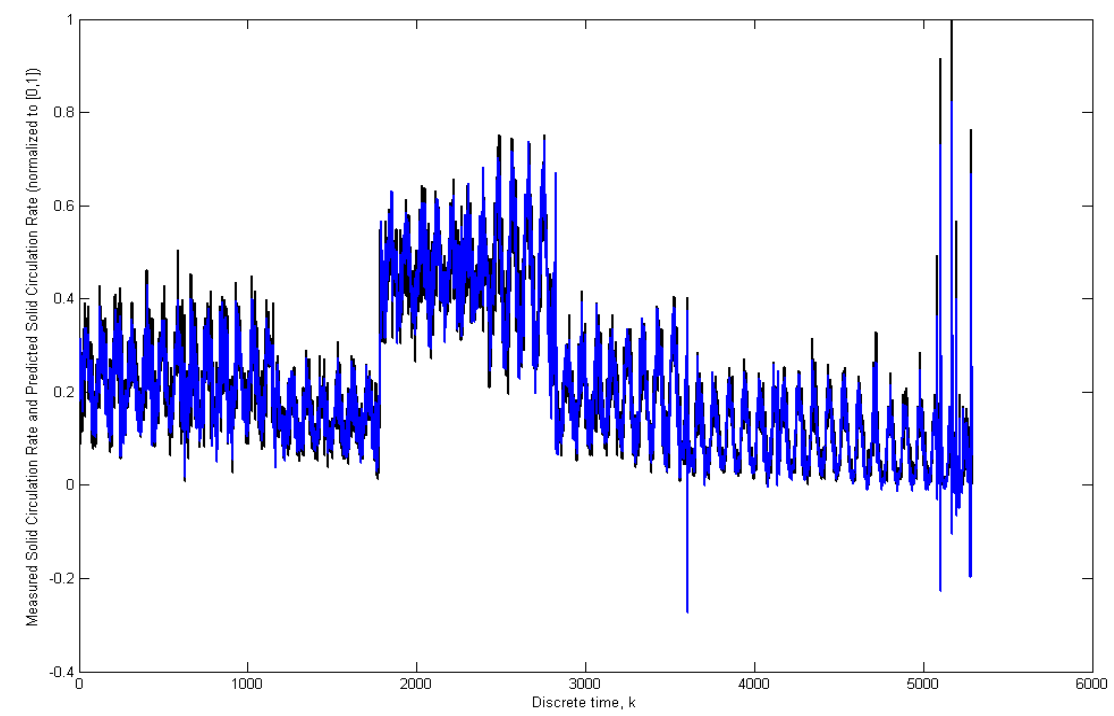

Figure 3.4 (d): Prediction of solids circulation rate using MMI, $\boldsymbol{s}=\mathbf{2}$ and $\boldsymbol{m o d e l}$ fit $=\mathbf{6 5 . 6 0} \%$. Blue line represents the predicted solids circulation rate and black line represents measured solids circulation rate. Solids circulation rate is normalized between $[0,1]$.

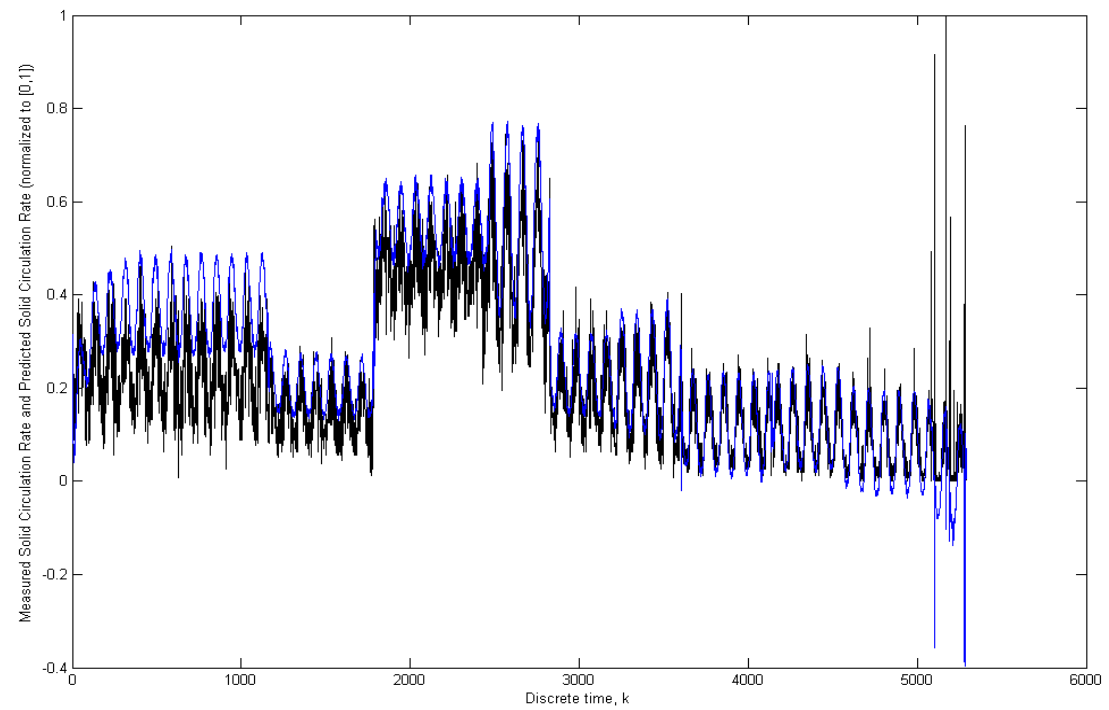

Figure 3.4 (e): Prediction of solids circulation rate using N4SID, $s=1$ and model fit $=\mathbf{3 1 . 8 0} \%$. Blue line represents the predicted solids circulation rate and black line represents measured solids circulation rate. Solids circulation rate is normalized between $[0,1]$.

Figure (3.4): BED MATERIAL - GLASS BEADS. Validation data set that comprises of riser aeration, modulated move air flow and the developed pressure drop across the riser as the inputs (taken in September 2006). Also shown is the prediction of solids circulation rate with the predictors defined in Eqs. (3.14) and (3.15). $\mathrm{X}$-axis is the discrete time index. 


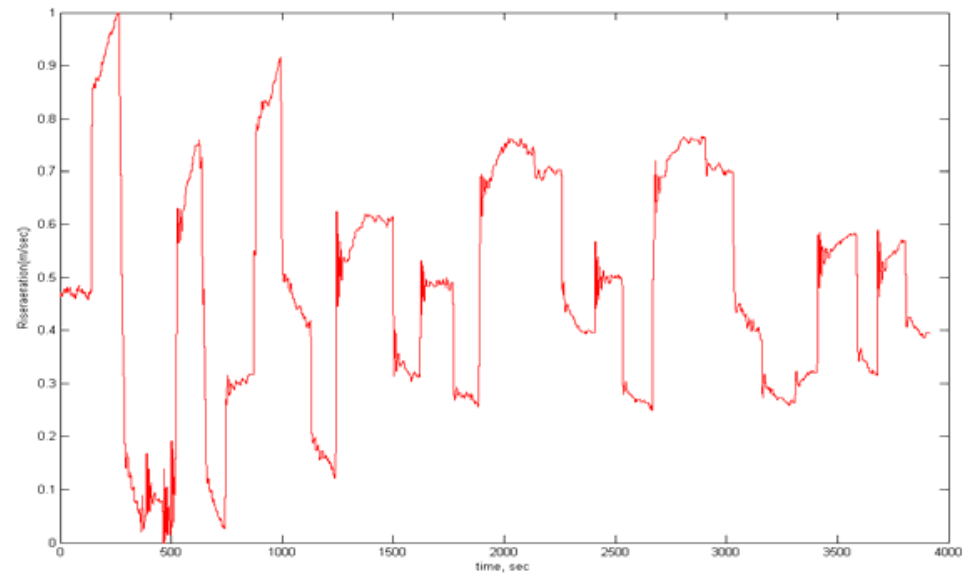

Figure $\quad 3.5 \quad$ (a): Riser aeration normalized between $[0,1]$.

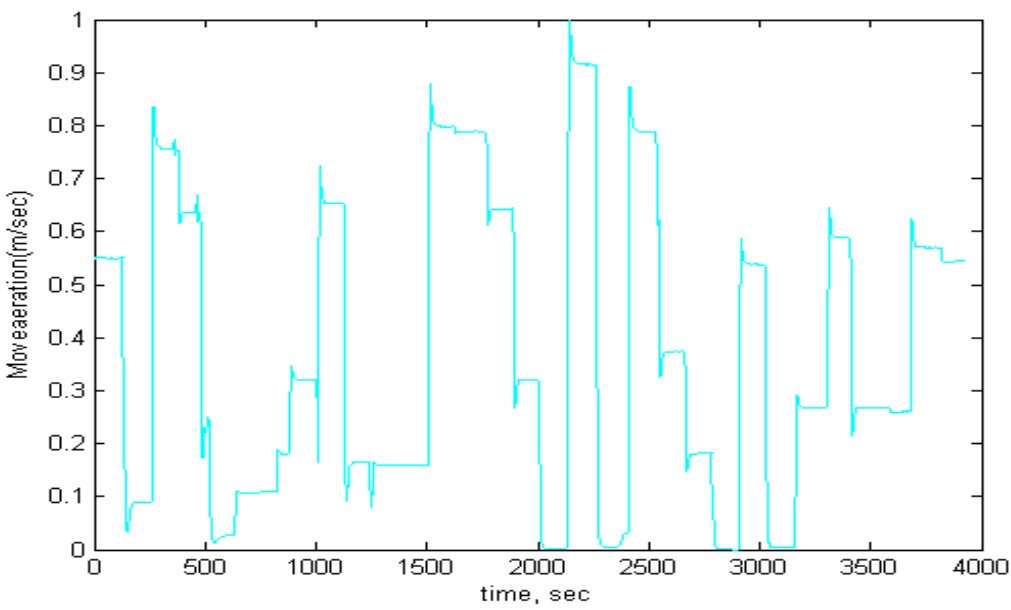

Figure 3.5 (b): Move air flow normalized between $[0,1]$.

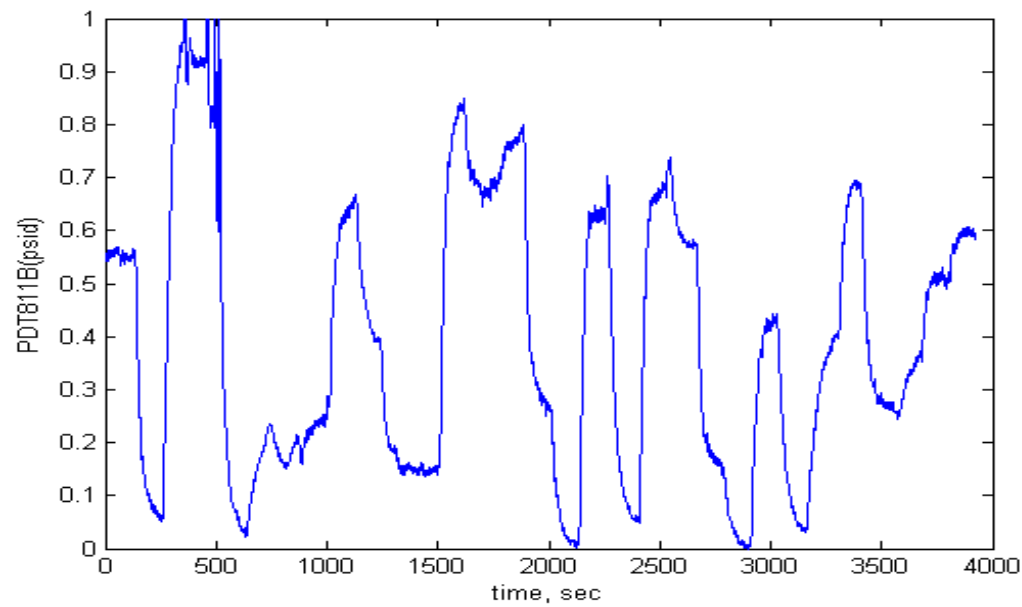

Figure 3.5 (c): Total riser pressure drop normalized between $[0,1]$.

Three inputs comprising of riser aeration, move air flow and total riser pressure drop, used to validate the predictors defined in Eq. (3.13) and Eq. (3.15). 


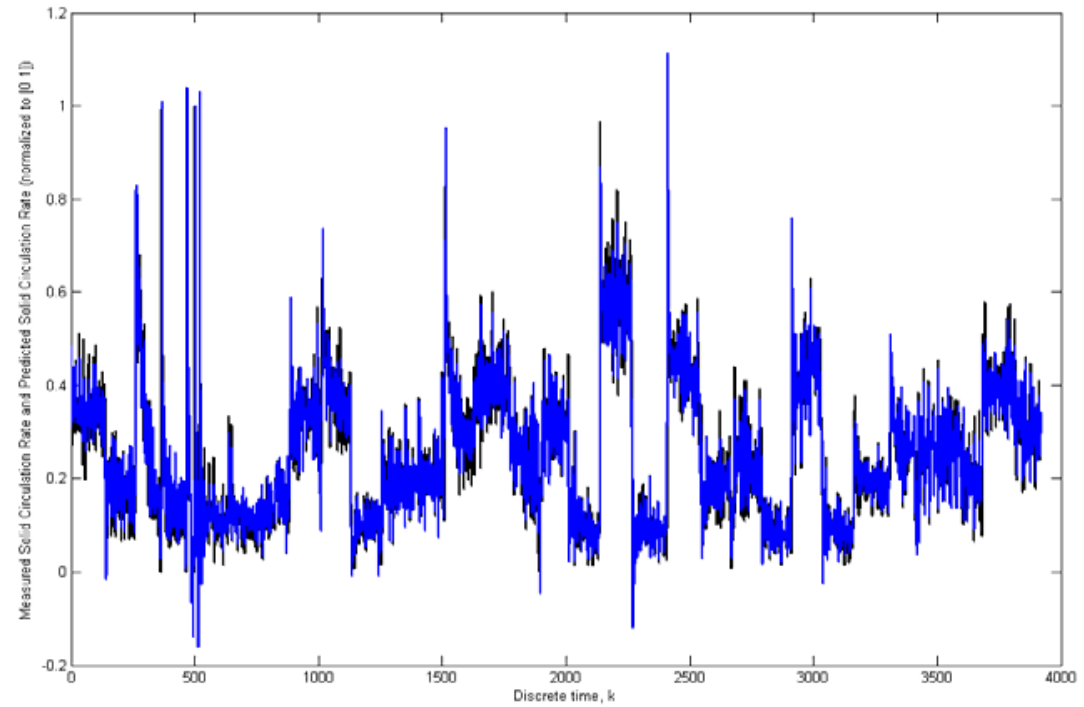

Figure 3.5 (d): Prediction of solids circulation rate using MMI, $\boldsymbol{s}=\mathbf{2}$ and $\boldsymbol{m o d e l}$ fit $=\mathbf{5 8 . 7 0} \%$. Blue line represents the predicted solids circulation rate and black line represents measured solids circulation rate. Solids circulation rate is normalized between $[0,1]$.

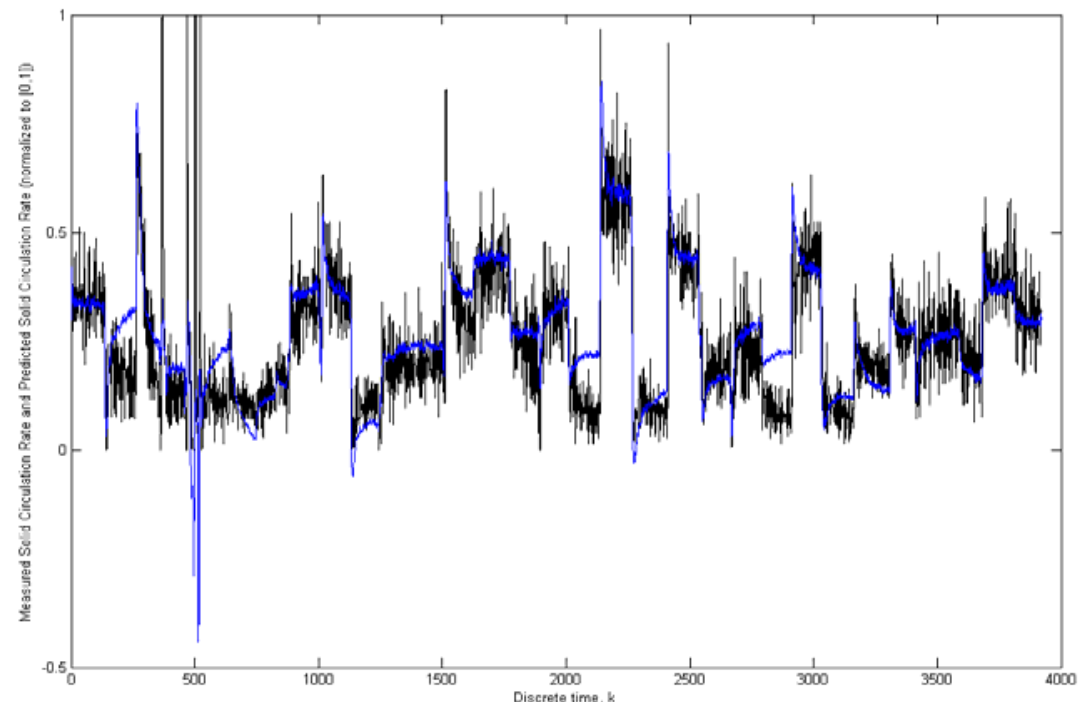

Figure 3.5 (e): Prediction of solids circulation rate using N4SID, $s=1$ and $\operatorname{model}$ fit $=\mathbf{3 6 . 8 3} \%$. Blue line represents the predicted solids circulation rate and black line represents measured solids circulation rate. Solids circulation rate is normalized between $[0,1]$.

Figure (3.5): BED MATERIAL - GLASS BEADS. Validation data set that comprises of random riser aeration and move air flow, both varied simultaneously and the developed pressure drop across the riser as the inputs (taken in October 2006). Also shown is the prediction of solids circulation rate with the predictors defined in Eqs. (3.14) and (3.15). X-axis is the discrete time index. 


\begin{tabular}{|c|c|c|}
\hline No. of Models in MMI & MMI Model fit & \multirow{2}{*}{ N4SID Model fit, $\boldsymbol{s}=\mathbf{I}$} \\
\hline$s=2$ & $58.70 \%$ & \multirow{2}{*}{$36.83 \%$} \\
\hline$s=3$ & $58.72 \%$ & \\
\hline$s=4$ & $58.32 \%$ & \\
\hline$s=5$ & $58.16 \%$ & \\
\hline
\end{tabular}

Table (3.4): BED MATERIAL - GLASS BEADS. Number of models in a multiple model approach and the corresponding model fits for the validation data set where both riser aeration and move air flow were varied randomly at the same time. Model fit for linear time invariant system obtained with N4SID algorithm is also shown.

The predictor generated using glass bead data is also validated against the cork data set that is obtained according to the experimental condition given in Table (3.5). When the model fits obtained with different numbers of models are compared, there is a slight improvement with five models over two models (Table (3.6)). However, the two model version can be used to avoid the complexity that may arise using higher number of models during control. The validation results for cork data sets are shown in Fig. (3.6). Thus, in vastly different experimental conditions (glass beads and cork bed materials), the one-step-ahead predictor obtained by training with the data sets that change randomly move air flow and riser aeration, one at a time, can predict well the solids circulation rate.

\begin{tabular}{|c|c|c|c|c|c|c|}
\hline Variable & \multicolumn{7}{|c|}{ Properties } \\
\hline Bed Material & \multicolumn{7}{|c|}{ Cork $\left(\boldsymbol{d}_{\boldsymbol{p}}=829 \mu \boldsymbol{\mu m}, \rho_{\boldsymbol{p}}=189 \mathbf{K g} / \mathbf{m}^{3}\right)$} \\
\hline Riser Aeration, (m/s) & 5.25 & 5.25 & 5.25 & 5.25 & 5.25 & 5.25 \\
\hline Move Air Flow (m/s) & 0.15 & 0.12 & 0.12 & 0.15 & 0.12 & 0.15 \\
\hline Period (s) & 90 & 60 & 90 & 120 & 120 & 60 \\
\hline
\end{tabular}

\begin{tabular}{|c|c|c|c|c|c|c|}
\hline Variable & \multicolumn{7}{|c|}{ Properties } \\
\hline Bed Material & \multicolumn{7}{|c|}{ Cork $\left(\boldsymbol{d}_{\boldsymbol{p}}=829 \mu \boldsymbol{m}, \rho_{\boldsymbol{p}}=189 \mathbf{K g} / \mathbf{m}^{\mathbf{3}}\right)$} \\
\hline Riser Aeration, (m/s) & 5.25 & 5.25 & 5.25 & 5.25 & 5.25 & 5.25 \\
\hline Move Air Flow (m/s) & 0.12 & 0.15 & 0.12 & 0.15 & 0.15 & 0.12 \\
\hline Period (s) & 90 & 120 & 120 & 90 & 60 & 60 \\
\hline
\end{tabular}

Table (3.5): Experimental condition at which move air flow was modulated. The experiment was performed with cork material in December, 2001. 

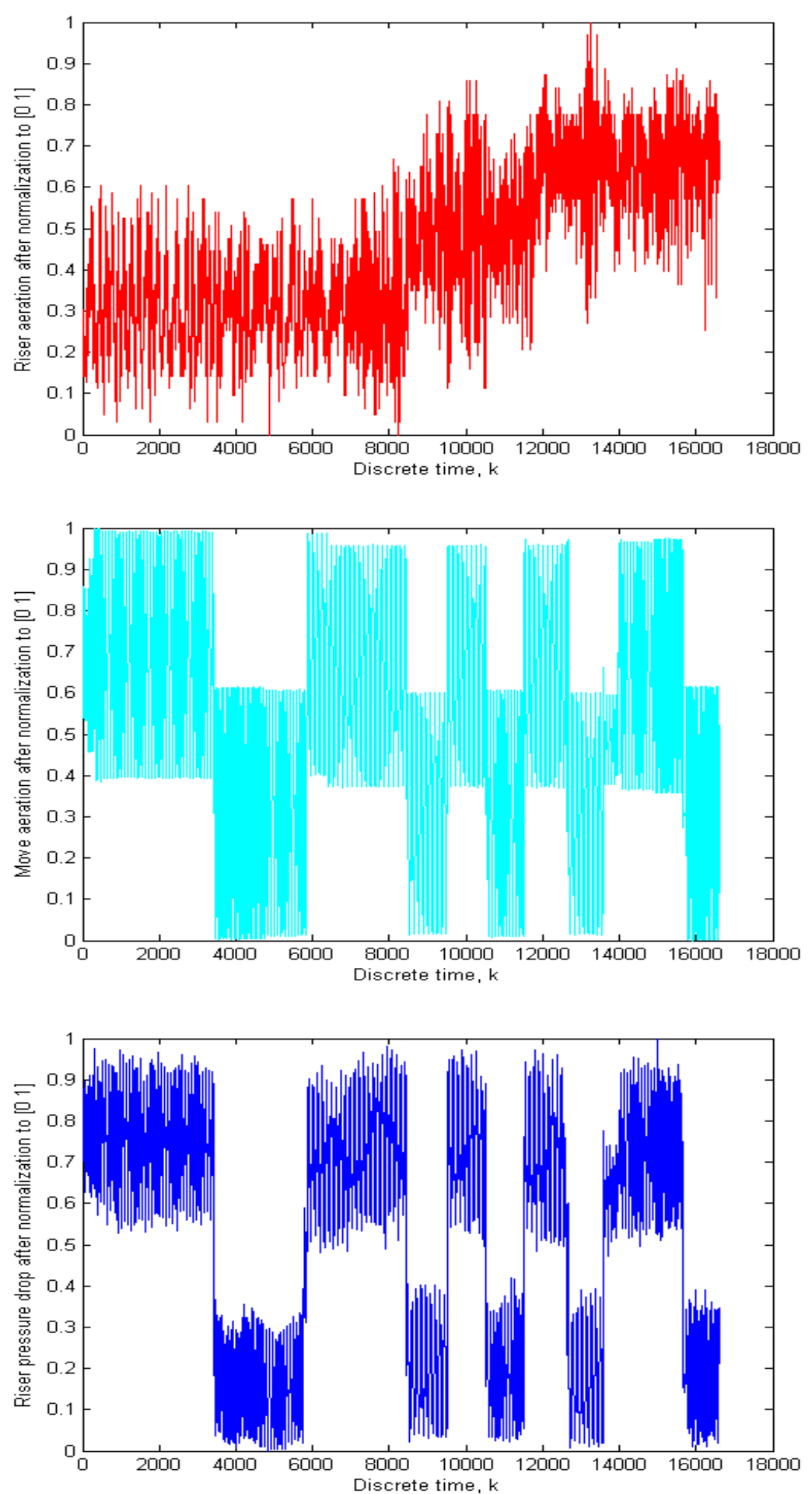

Figure $3.6 \quad$ (a): Riser aeration normalized between $[0,1]$.

Figure 3.6 (b): Move air flow normalized between $[0,1]$.
Figure 3.6 (c): Total riser pressure drop normalized between $[0,1]$.

Three inputs comprising of riser aeration, move air flow and total riser pressure drop, used to validate the predictors defined in Eq. (3.13) and Eq. (3.15). 


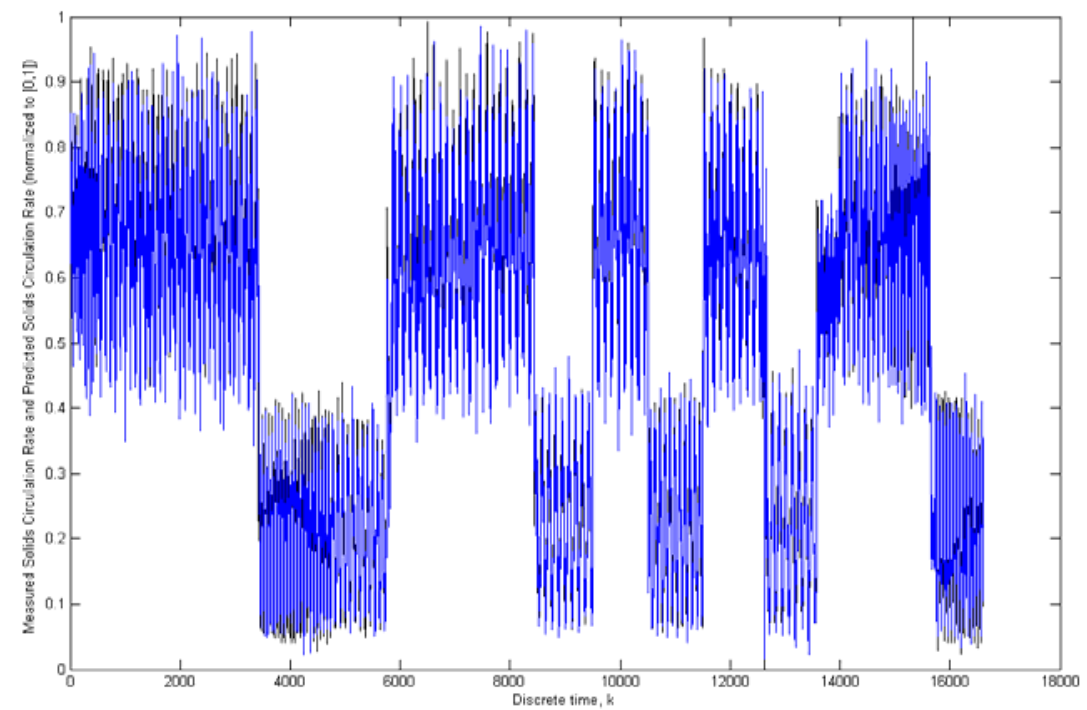

Figure 3.6 (d): Prediction of solids circulation rate using MMI, $s=2$ and model fit $=\mathbf{7 1 . 4 9} \%$. Blue line represents the predicted solids circulation rate and black line represents measured solids circulation rate. Solids circulation rate is normalized between $[0,1]$.

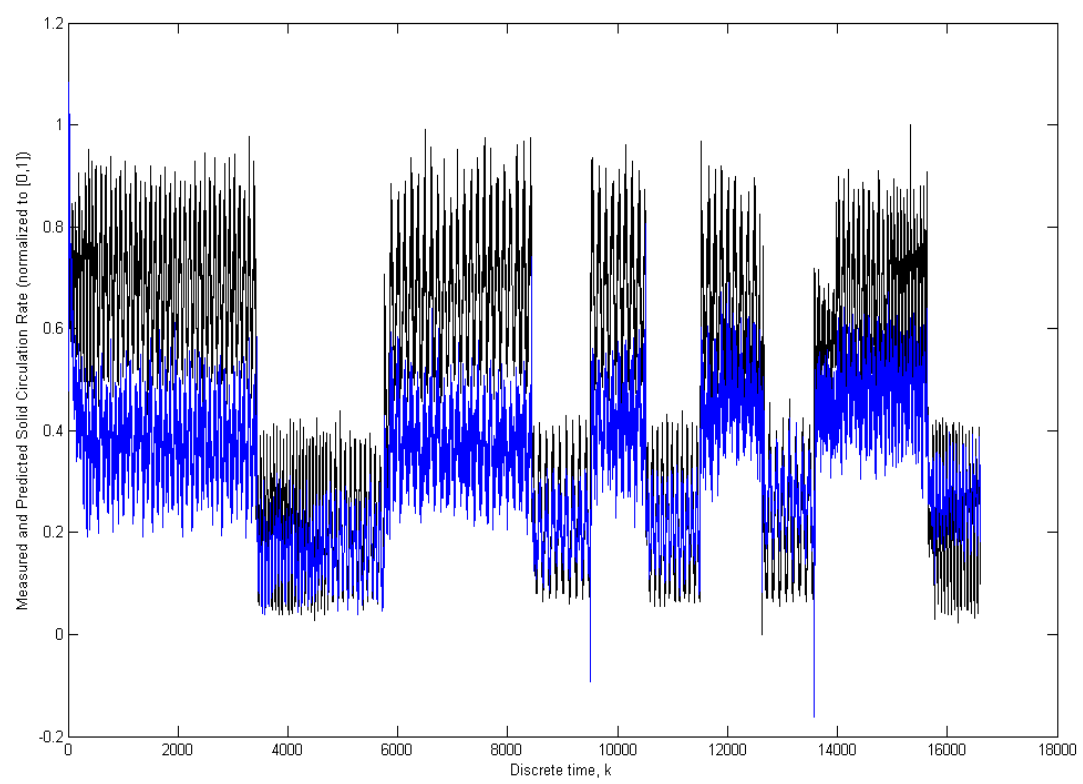

Figure 3.6 (e): Prediction of solids circulation rate using N4SID, $s=1$ and model fit $=7.41 \%$. Blue line represents the predicted solids circulation rate and black line represents measured solids circulation rate. Solids circulation rate is normalized between $[0,1]$.

Figure (3.6): BED MATERIAL - CORK. Validation data set that comprises of riser aeration modulated move air flow and the developed pressure drop across the riser as the inputs (taken in December 2001). Also shown is the prediction of solids circulation rate with the predictors defined in Eqs. (3.14) and (3.15). X-axis is the discrete time index. 


\begin{tabular}{|c|c|c|}
\hline No. of Models in MMI & MMI Model fit & \multirow{2}{*}{ N4SID Model fit, $\boldsymbol{s}=\mathbf{I}$} \\
\hline$s=2$ & $71.46 \%$ & \multirow{2}{*}{$7.41 \%$} \\
\hline$s=3$ & $71.98 \%$ & \\
\hline$s=4$ & $71.96 \%$ & \\
\hline$s=5$ & $73.30 \%$ & \\
\hline
\end{tabular}

Table (3.6): BED MATERIAL - CORK. Number of models in a multiple model approach and the corresponding model fits for the validation data set where move air flow was modulated. Model fit for linear time invariant system obtained with N4SID algorithm is also shown.

The SCR results from the global linear model estimated by N4SID are presented in Figs. 2.3(e), 2.4(e) and 2.5(e) for glass beads and Fig. 2.6(e) for cork. The model fits for the N4SID results are respectively $36.57 \%, 31.8 \%, 36.83 \%$ and $7.41 \%$. They can be compared to the SCR predictions for the two model case presented in Figs. 2.3(d), 2.4(d), 2.5(d), and 2.6(d), where model fits are respectively, 63.0\%, 65.6\%, $58.7 \%$ and $71.49 \%$. There is an appreciable increase in model fit with two local state space models than the global linear model estimated by the N4SID.

In this section, identification based on a multiple model approach is used to obtain a predictor model for the cold flow circulating fluidized bed that is considered as a black box nonlinear dynamic system. The data set is divided into identification and validation data sets. The number of models is increased from $s=2$ to $s=5$ for the glass beads data set (training data set) that is obtained by randomly varying riser aeration and move air flow one at a time and two third order models give appreciable improvement in the model fit for the different validation data sets. The comparison of the model fits between the results obtained from N4SID and multiple model approach for the given data sets and the experimental results are also presented. 


\section{Chapter 4}

\section{Linear System Identification of a Cold Flow Circulating Fluidized Bed}

\subsection{Motivation}

The motivation behind the separate analysis of deterministic and stochastic components of any physical variable of interest within the given system urges one to develop a model that pursues this goal. This helps to analyze if the statistical description of a certain variable follows a specific distribution such that this inference could be made for the measurement errors in future. Similarly, when the system operates under a particular regime, it would be helpful to understand if there exists a certain relationship between dependent and independent variables. The presence of noise in measurements makes the task difficult and hence, the separation of measured quantities into stochastic and deterministic elements helps to deduce this relationship.

A theory has been developed that starts out with a complete black-box modeling and converts this model to the grey box model that does not include any fictitious variable so that the interaction between the physical parameters within the given system can then be analyzed and interpreted. Separate noise and deterministic models have been deduced for both cases when the order of intermediate state equals or is greater than the number of outputs used. The stochastic and deterministic components of these variables are then separated; this separation lacks in any of the works that have been carried out using either system identification or physical modeling of the CFCFB, till date.

\subsection{Linear System Identification: Basic Theory}

This chapter deals with the identification of CFCFB, considering it as a discrete-time linear time invariant (LTI) system while operating under dilute transport regime. A state-space description of a discrete-time LTI system has the form:

$x(k+1)=f\left(x_{k}, u_{k}\right)$

$y(k)=g\left(x_{k}, u_{k}\right)$

where $x_{k} \in \mathfrak{R}^{n x l}$ is the state of the system, $u_{k} \in \mathfrak{R}^{m x 1}$ the input, $y_{k} \in \mathfrak{R}^{\ell x 1}$ the output and $f$ and $g$ are linear vector functions. The dimension $n$ of the state vector is called the order of the system. The order indicates the memory that is present in the system, and has an influence on the time behavior of the system. The variable $k$ is the discrete time index.

With regard to the CFCFB, the input vector $u_{k}$ consists of move air flow, riser aeration and loop seal fluidization air. The state vector $x_{k}$ has no physical meaning associated with the CFCFB and the order of the system is not known a priori. Hence, Eq. (4.1) is a black-box representation of a given system.

Hence, instead of using a state-space description (4.1) of a system that includes a fictitious vector $x_{k}$, the given system can be implemented in the input-output form as presented in Chapter 3 where the relation includes only known terms. However, all those advantages that arise due to the use of state space 
description as discussed in that chapter amount to a rational behind choosing the state space description for the CFCFB.

Move air flow, aeration injected into the standpipe just above the entrance of the non-mechanical valve, combined with aeration supplied to the non-mechanical valve controls the solids circulation rate. When the move air flow is changed, the solids circulation rate changes. Changing the solids circulation rate shifts the split of inventory between the riser and the standpipe which, in turn, changes the bed height in the standpipe. Move air flow is under the control of the user and hence, it is chosen as one of the inputs in the determination of the model through the prediction error method. Other inputs include riser aeration injected upwards at the base of the riser and the loop seal fluidization air. Outputs are the pressure drops across the CFCFB such as the total pressure drop across the riser, the pressure drop across the crossover, the pressure drop across the cyclone, the total pressure drop across the stand pipe, the pressure drop across the loop seal and the measured SCR during identification phase.

For the CFCFB, the pressure balance is Rhodes et. al.[37]:

$$
\Delta P_{\text {valve }}+\Delta P_{\text {riser }}+\Delta P_{\text {crossover }}+\Delta P_{\text {cyclone }}=\Delta P_{\text {stan dpipe }}
$$

where $\Delta P_{\text {valve }}$ is the pressure drop around loop seal to the base of the riser, $\Delta P_{\text {riser }}$ is the pressure drop across the riser, $\Delta P_{\text {crossover }}$ is the pressure drop across the crossover, $\Delta P_{\text {cyclone }}$ is the pressure drop across the primary cyclone and $\Delta P_{s \tan \text { dpipe }}$ is the pressure drop across the stand pipe.

The pressure drop across the riser must be balanced by the pressure drop across the stand pipe. This balancing pressure drop is generated by gas flows up through the two parts of the equipment. If the circulation rate is increased, then the inventory is shifted from the stand pipe and into the riser and hence, increases the riser pressure drop $\Delta P_{\text {riser }}$. The higher $\Delta P_{\text {riser }}$ in the riser combined with the lower amount of solids in the stand pipe (note that the increased solids in the riser comes from the stand pipe) causes more gas to flow upward through the stand pipe. The higher gas flow results in a higher pressure drop across the packed bed of the stand pipe. The gas flow increases until the stand pipe and the riser $\Delta P_{\text {riser }}$ are in balance again. However, there is a limit to the amount of gas which can flow up through the stand pipe. The maximum amount of $\Delta P_{s \tan \text { dpipe }}$ in the stand pipe is fixed by the minimum fluidization velocity of the bed material. Once the minimum fluidization velocity has been reached, then the pressure drop across the bed is constant and the inventory would be shifted back into the stand pipe from the riser until the system was back in pressure balance.

A fact that is very closely related to the above discussion should be remembered. The intent of the CFB is to have gas flow upward through the riser and solids flow from the standpipe into the riser. If the pressure balance gets too far out of balance, then the primary function of the CFB is significantly disrupted with major gas flow upward through the stand pipe.

Second, because the experiment is really a closed system with respect to the bed material (that is to say those solids do not continuously enter or leave the system), then the amount of material in the riser and standpipe during operation is usually present in the standpipe before the circulation is begun. The maximum amount of material that can be easily accommodated in the system "at rest" has the stand pipe full and solids sitting in the primary cyclone. After solids circulation begins, the total amount of material splits between the riser and the stand pipe such that the stand pipe bed height is observed within certain 
limits. The variation in the stand pipe bed height depends on the circulation rate and the gas flow through the riser.

Thus, with the above observation of this interrelation among these variables under the action of independent stand pipe and riser aerations, intuition suggests choosing them as the outputs $(y(k))$ of the system under the influence of these gas flows.

With the Taylor series expansion of Eq. (4.1) at the operating point $\left(\left(x_{e}, u_{e}\right) \equiv(0,0)\right)$ and neglecting higher order terms other than the first order of the expansion, we obtain the following system:

$x(k+1)=A x(k)+B u(k)$

$y(k)=C x(k)$

with the proper definition of $A \in \mathfrak{R}^{n x n}, B \in \mathfrak{R}^{n x m}$ and $C \in \mathfrak{R}^{l x n}$, Kailath [38].

In a real system like the NETL CFCFB, the measurements of physical variables are always contaminated with noise. If it is assumed that the measurement noise $e(k) \in \mathfrak{R}^{l x l}$ propagates to the deterministic dynamics of the system (3.3) through a matrix $K \in \mathfrak{R}^{n x l}$, the plant can be defined as:

$$
\begin{aligned}
& x(k+1)=A x(k)+B u(k)+K e(k) \\
& y(k)=C x(k)+e(k)
\end{aligned}
$$

The term $e(k) \in \mathfrak{R}^{l x l}$ is also called the "innovation" that represents that part of the output $y(k)$ that cannot be predicted from the past data Ljung[25] and hence, the plant is said to be defined in "innovation" form.

The one-step-ahead predictor for the CFCFB can then be defined as:

$$
\begin{aligned}
& \hat{x}(k+1)=\hat{A} \hat{x}(k)+\hat{B} u(k)+\hat{K}(y(k)-\hat{y}(k)) \\
& \hat{y}(k)=\hat{C} \hat{x}(k)
\end{aligned}
$$

where $\hat{y}(k)$ is the estimated output vector, $\hat{A} \in \mathfrak{R}^{n x n}, \hat{B} \in \mathfrak{R}^{n x m}, \hat{K} \in \mathfrak{R}^{n x l}$ and $\hat{C} \in \mathfrak{R}^{l x n}$ are the estimates of the system matrices which need to be determined from the input-output data available from the plant and $\hat{x}(k) \in \mathfrak{R}^{n x l}$ is the state estimate.

\subsection{Prediction Error Method}

As discussed in Chapter 3, system identification through the prediction error method is carried out by minimizing the difference between the output of a model (4.5) and the measured output of the system (4.4). The Gauss Newton algorithm is used to minimize the prediction-error cost function. Subspace method N4SID is used to generate an initial starting point for an iterative prediction-error method. The desired order of the system is determined through the N4SID routine in the System Identification Toolbox present in MATLAB. 


\subsection{New System Model for the CFCFB: Grey Box Model}

Now, it is clear that CFCFB is described in terms of a fictitious state vector $x_{k}$, it is quite reasonable to arrive at a model that does not include any such unknown terms and is defined only in terms of physical parameters of the given system. To start with, the system (4.4) is split up into deterministic and stochastic subsystems, by splitting up the state $x(k)$ and the output $y(k)$ in a deterministic $\left({ }^{\mathrm{d}}\right)$ and stochastic $\left(.{ }^{\mathrm{s}}\right)$ components: $x(k)=x^{d}(k)+x^{s}(k), y(k)=y^{d}(k)+y^{s}(k)$. The deterministic state $\left(x^{d}(k)\right)$ and output $\left(y^{d}(k)\right)$ follow from the deterministic subsystem, which describes the influence of the deterministic input $(u(k))$ on the deterministic output:

$x^{d}(k+1)=A x^{d}(k)+B u(k)$

$y^{d}(k)=C x^{d}(k)$

The stochastic state $\left(x^{s}(k)\right)$ and output $\left(y^{s}(k)\right)$ follow from the stochastic subsystem, which describes the influence of the unmeasurable components including process and measurement noises on the stochastic output:

$$
\begin{aligned}
& x^{s}(k+1)=A x^{s}(k)+K e(k) \\
& y^{s}(k)=C x^{s}(k)+e(k)
\end{aligned}
$$

When the matrices $\hat{A}, \hat{B}, \hat{K}$, and $\hat{C}$ are identified using prediction-error method on the one-step-ahead predictor defined in Eq. (4.5), the innovation term $e(k)$ is obtained from Eq. (4.4). In this research, the measured values of solids circulation rate are used only in the determination of $\hat{A}, \hat{B}, \hat{K}$, and $\hat{C}$. With the point made out in the introduction, that the solids circulation rate is not measurable in the extreme process conditions, it is required to obtain the stochastic estimate of SCR from the pressure drops across the CFCFB. Hence, using the transformation $z(k)=M y(k)$ where $M=I_{(l-l) x l}, I$ : Identity matrix, the system (4.4) becomes

$$
\begin{aligned}
& \bar{e}(k)=z(k)-M \hat{C} x(k) \\
& x(k+1)=\hat{A} x(k)+\hat{B} u(k)+\hat{K} T \bar{e}(k)
\end{aligned}
$$

where $\bar{e}(k) \in \mathfrak{R}^{(l-I) x l}=M e(k)$ is the innovation vector for pressure drops across the CFCFB determined from the measured values of these pressure variables $(z(k))$ and $T \in \mathfrak{R}^{\ell x(\ell-1)}$ is the pseudoinverse of $M$. The stochastic SCR is then obtained from

$$
\begin{aligned}
& x^{s}(k+1)=\hat{A} x^{s}(k)+\hat{K} T \bar{e}(k) \\
& \hat{y}^{s}(k)=\hat{C} x^{s}(k)+T e(k)
\end{aligned}
$$

It is clear that for the pressure drops across the CFCFB, the innovation term contains that part of these parameters that cannot be predicted by the model. Hence, combining the stochastic pressure drops and deterministic estimate, the model response for these variables turns out to be as exact as those produced by the plant itself, that is, the modelfit (defined in the next section) between the estimated and measured pressure drops comes out to be $100 \%$. Such results would make no sense since the innovation terms for 
these pressure drops were obtained by the estimation of that part of their measurements obtained from the CFCFB that could not be predicted by the model and then adding the ensuing stochastic response from these innovation vectors and the perfect deterministic estimate. A similar observation could be made for the solids circulation rate as well if the innovation term is computed for the solids circulation rate from its measurement (even though it can be done in the present case with the offline data set at hand, it has not been performed for computation except for comparison purpose only) and then combining the stochastic and deterministic estimate. Instead, the SCR stochastic response is obtained from the noise model utilizing the innovation terms for the pressure drops only rather than computing its own innovation term from its measurements and utilizing it in the noise model. Consequently, this would lead to the computation of the real time stochastic response for the solids circulation rate from the stochastic pressure drops during control action in which case the actual measured solids circulation rate of the plant is not required while using the noise model.

As mentioned above, the order of the system needs to be determined from the input-output data only, it can be either $n=\ell$ or $n>\ell$. The standing assumption during the identification phase is that the system (4.4) is stable, controllable and observable, [25]. The observability condition leads to the fact that the state matrix $\hat{A}$ and the output matrix $\hat{C}$ are invertible when $n=\ell$. For the $n=\ell$ case, a new model can be obtained by the output-to-state transformation $x^{d}(k)=\hat{C}^{-1} y^{d}(k)$ and $x^{s}(k)=\hat{C}^{-1}\left(y^{s}(k)-T e(k)\right)$. The new state vectors $y^{d}(k)$ and $y^{s}(k)$ contain deterministic and stochastic pressure drops and solids circulation rate, respectively and the new output vectors $z^{d}(k) \in \mathfrak{R}^{(\ell-1) x l}=M y^{d}(k)$ and $z^{s}(k) \in \mathfrak{R}^{(\ell-1) x l}=M y^{s}(k)+\bar{e}(k)$ are defined in terms of pressure drops only.

$y^{d}(k)=A_{\text {new }} y^{d}(k-1)+B_{\text {new }} u(k-1)$

$z^{d}(k)=M y^{d}(k)$

$y^{s}(k)=A_{\text {new }} y^{s}(k-1)+K_{n e w} T \bar{e}(k-1)+T e(k)$

$z^{s}(k)=M y^{s}(k)+\bar{e}(k)$

with $A_{\text {new }}=\hat{C} \hat{A} \hat{C}^{-1}, B_{\text {new }}=\hat{C} \hat{B}$ and $K_{\text {new }}=\hat{C}\left(\hat{K}-\hat{A} \hat{C}^{-1}\right)$. Thus, the new system (4.10) is defined in terms of physical parameters of CFCFB only. The different pressure drops across the cold set up and the solids circulation rate are linearly related to each other under the influence of aeration flows fed across various section of the machine.

On the other hand, when $n>\ell$, the model order of deterministic and stochastic subsystems (4.6) and (4.9) needs to be reduced such that $n=\ell$. This is the more general case and hence, the derivation is discussed in the separate sub-section.

\section{4.a Model Order Reduction when $n>l$}

Model order reduction is achieved by deleting states in I/O balancing realization, Laub et. al.[39], Moore[40], and Laub[41]. The state coordinate transformation $\bar{x}^{d}=Q_{d} x^{d}$ or $\bar{x}^{s}=Q_{s} x^{s}$ produces the equivalent model

$$
\begin{aligned}
& \bar{x}^{d}(k+1)=Q_{d} \hat{A} Q_{d}^{-l} \bar{x}^{d}(k)+Q_{d} \hat{B} u(k) \\
& y^{d}(k)=\hat{C} Q_{d}^{-l} \bar{x}^{d}(k)
\end{aligned}
$$


$\bar{x}^{s}(k+1)=Q_{s} \hat{A} Q_{s}^{-1} \bar{x}^{s}(k)+Q_{s} \hat{K} T \bar{e}(k)$

$\hat{y}^{s}(k)=\hat{C} Q_{s}^{-l} \bar{x}^{s}(k)+T \bar{e}(k)$

and transforms the grammians to $\bar{W}_{c}^{d}=Q_{d} W_{c}^{d} Q_{d}^{T}, \bar{W}_{o}^{d}=Q_{d}^{-T} W_{o}^{d} Q_{d}^{-1} \quad$ or $\bar{W}_{c}^{s}=Q_{s} W_{c}^{s} Q_{s}^{T}, \bar{W}_{o}^{s}=Q_{s}^{-T} W_{o}^{s} Q_{s}^{-l}$ where the controllability and observability grammians $W_{c}^{d}, W_{o}^{d}$ or $W_{c}^{s}, W_{o}^{s}$ are defined as

$$
\begin{array}{ll}
W_{c}^{d}=\sum_{k=0}^{\infty} \hat{A}^{k} \hat{B} \hat{B}^{T}\left(\hat{A}^{T}\right)^{k}, & W_{o}^{d}=\sum_{k=0}^{\infty}\left(\hat{A}^{T}\right)^{k} \hat{C}^{T} \hat{C} \hat{A}^{k} \\
W_{c}^{s}=\sum_{k=0}^{\infty} \hat{A}^{k}(\hat{K} T)(\hat{K} T)^{T}\left(\hat{A}^{T}\right)^{k}, & W_{o}^{s}=\sum_{k=0}^{\infty}\left(\hat{A}^{T}\right)^{k} \hat{C}^{T} \hat{C} \hat{A}^{k}
\end{array}
$$

The transformation $Q_{d}$ and $Q_{s}$ are computed such that $\bar{W}_{c}^{d}=\bar{W}_{o}^{d}=\operatorname{diag}\left(g_{d}\right)$ and $\bar{W}_{c}^{s}=\bar{W}_{o}^{s}=\operatorname{diag}\left(g_{s}\right)$, respectively, where $g_{d}$ or $g_{s}$ reflects the combined controllability and observability of individual states of the balanced model. Hence, these states with small $g_{d}(i), i=1, \ldots, n$ or $g_{s}(i), i=1, \ldots, n$ can be deleted until $n=\ell$ while retaining the most important input-output characteristics of the original system.

In the present case, the full state vector $x^{d}(k)$ or $x^{s}(k)$ is partitioned as $x^{d}(k)=\left[x_{\ell}^{d}(k) \quad x_{n-\ell}^{d}(k)\right]^{T}$ or $x^{s}(k)=\left[\begin{array}{ll}x_{\ell}^{s}(k) & x_{n-\ell}^{s}(k)\end{array}\right]^{T}$ where $x_{n-\ell}^{d}(k)$ or $x_{n-\ell}^{s}(k)$ is to be discarded, and the reduced state is set to $x_{r=\ell}^{d}(k)=x_{\ell}^{d}(k)+R_{d} x_{n-\ell}^{d}(k)$ or $x_{r=\ell}^{s}(k)=x_{\ell}^{s}(k)+R_{s} x_{n-\ell}^{s}(k)$ where $R_{d}$ or $R_{s}$ is chosen to enforce matching DC gains (steady state response) between original subsystems and reduced subsystems. The matched DC gain method is performed by setting $x_{n-\ell}^{d}(k+1)=x_{n-\ell}^{d}(k)$ or $x_{n-\ell}^{s}(k+1)=x_{n-\ell}^{s}(k)$ and solving the resulting equation for $x_{\ell}^{d}(k)$ or $x_{\ell}^{s}(k)$. The state vectors $x_{n-\ell}^{d}(k)$ or $x_{n-\ell}^{s}(k)$ are discarded because they are assumed to possess smaller values of $g_{d}$ or $g_{s}$, respectively.

The resulting deterministic and stochastic subsystems become

$$
\begin{aligned}
& x_{\ell}^{d}(k+1)=\hat{A}_{\ell x \ell}^{d} x_{\ell}^{d}(k)+\hat{B}_{\ell x m}^{d} u(k) \\
& y^{d}(k)=\hat{C}_{\ell x \ell}^{d} x_{\ell}^{d}(k)+\hat{D}_{\ell x m}^{d} u(k) \\
& x_{\ell}^{s}(k+1)=\hat{A}_{\ell x \ell}^{s} x_{\ell}^{s}(k)+\hat{K}_{\ell x(\ell-1)}^{s} \bar{e}(k) \\
& y^{s}(k)=\hat{C}_{\ell x \ell}^{s} x_{\ell}^{s}(k)+\hat{D}_{\ell x(\ell-1)}^{s} \bar{e}(k)
\end{aligned}
$$

Here, the state vectors still bear no physical meaning of any system variables and hence, with the outputto-state transformation as in the case of $n=\ell$, we again obtain the following grey box model of the CFCFB, where there again exists a linear relationship between the various pressure drops and solids circulation rate under the action of the aeration flows. 
$y^{d}(k)=A_{\text {new }}^{d} y^{d}(k-1)+B_{\text {new }}^{d} u(k-1)+\hat{D}_{\ell x m}^{d} u(k)$

$z^{d}(k)=M y^{d}(k)$

where $A_{\text {new }}^{d}=\hat{C}_{\ell x \ell}^{d} \hat{A}_{\ell x \ell}^{d}\left(\hat{C}_{\ell x \ell}^{d}\right)^{-1}, B_{\text {new }}^{d}=\hat{C}_{\ell x \ell}^{d}\left(\hat{B}_{\ell x m}^{d}-\hat{A}_{\ell x \ell}^{d}\left(\hat{C}_{\ell x \ell}^{d}\right)^{-1} \hat{D}_{\ell x m}^{d}\right)$

$y^{s}(k)=A_{\text {new }}^{s} y^{s}(k-1)+K_{\text {new }}^{s} \bar{e}(k-1)+\hat{D}_{\ell x(\ell-1)}^{s} \bar{e}(k)$

$z^{s}(k)=M y^{s}(k)+\bar{e}(k)$

where $A_{\text {new }}^{s}=\hat{C}_{\ell x \ell}^{s} \hat{A}_{\ell x \ell}^{s}\left(\hat{C}_{\ell x \ell}^{s}\right)^{-1}, K_{n e w}^{s}=\hat{C}_{\ell x \ell}^{s}\left(\hat{K}_{\ell x(\ell-1)}^{s}-\hat{A}_{\ell x \ell}^{s}\left(\hat{C}_{\ell x \ell}^{s}\right)^{-1} \hat{D}_{\ell x(\ell-1)}^{s}\right)$. Thus, as in the case of $n=\ell$, the new system(4.14) and (4.15) are also defined in terms of physical parameters of CFCFB only. The only difference with system (4.10) is that the system matrices for deterministic and stochastic subsystems are different as a consequence of model order reduction. In either case, the new state-space equations are defined at present times, as a function of inputs and states at previous time step.

\subsection{Results and Discussions}

The experimental data used in this work were obtained during operations where the following parameters were varied, Huang[13] and Table (3.5):

$F_{m}$ : Flow rate of move air, which has two levels $(0.15$ or $0.25 \mathrm{~m} / \mathrm{s})$.

Period: Period of sine function of move air's flow rate, which has three levels (60, 90 or 120 seconds). $U_{g}$ : Riser aeration that is fixed at $5.25 \mathrm{~m} / \mathrm{s}$.

$F_{l p s l}$ a $:$ Loop seal fluidization air that is fixed at $0.25 \mathrm{~m} / \mathrm{s}$.

The physical data and constants are listed as follows:

Inside cross-sectional area of standpipe: $A_{s}=0.05067 \mathrm{~m}^{2}$.

Inside cross-sectional area of riser: $A_{r s}=0.07296 \mathrm{~m}^{2}$.

Density of particles: $\rho_{P}=189 \mathrm{Kg} / \mathrm{m}^{3}$.

Density of gas: $\rho_{g}=1.22 \mathrm{Kg} / \mathrm{m}^{3}$ (air at $15^{\circ} \mathrm{C}$ and $1 \mathrm{~atm}$ ).

Viscosity of gas: $\mu=1.8 \times 10^{-5}$ Pa.s.

Particle sphericity: $\psi=0.84$.

Surface-volume diameter of particles: $d_{s v}=8.12 \times 10^{-4} \mathrm{~m}$.

Particle terminal velocity: $U_{t}=0.81 \mathrm{~m} / \mathrm{s}$.

The stochastic response of the SCR and that of the deterministic model are added together to obtain the overall estimate of the SCR. The estimate can be compared with the measured SCR in terms of modelfit defined as:

Modelfit $=\left(1-\frac{\left\|y_{S C R}(k)-\hat{y}_{S C R}(k)\right\|_{2}}{\left\|y_{S C R}(k)-\operatorname{mean}\left(y_{S C R}(k)\right)\right\|_{2}}\right) \times 100 \%, k=1, \ldots, N$

where, $y_{S C R}(k)$ is the measured SCR, $\hat{y}_{S C R}(k)$ is the estimated SCR. 
The initial states $x(0), x^{d}(0)$ and $x^{s}(0)$ are all taken as 0 . During the identification phase, when the order of the CFCFB is estimated from the singular values of the Hankel matrices of the impulse response, the default order comes out to be greater than 50 for the analyzed data set. However, the degree of excitation of the inputs comprising of move air flow $u_{1}$, riser aeration $u_{2}$ and loop seal fluidization air $u_{3}$, that is, the order of a model that the individual inputs are capable of estimating in an unambiguous way (persistently exciting condition of the inputs required for identification) is only [ 505050$]$. Hence, the order of the CFCFB model is determined using trial and error and the order of 7 was found to be a good choice in the one-step-ahead predictor identification. Model order reduction is therefore used to arrive at a grey box model of the CFCFB.

For estimation and validation of the model, both inputs and outputs are normalized to a $[0,1]$ range with the following equations:

$$
u_{\text {normalized }}=\frac{u-\min (u)}{\max (u)-\min (u)}
$$

and

$y_{\text {normalized }}=\frac{y-\min (y)}{\max (y)-\min (y)}$

Then, both the input and output measurements are detrended to remove linear trends from them. When the data has been collected from a physical plant, it is typically measured in physical units. The levels in these raw input and output measurements might not match in any consistent way. This will force the models to waste some parameters correcting the levels. The aeration flows in CFCFB were measured in Standard cubic feet per hour during operation where as the solids circulation rate was measured in pounds per hour. To derive a present model, aeration flows were converted to meters per second to comply with gas velocities standards and the solids circulation rate was converted to $\mathrm{Kg}$ per second.

\section{5.a Validation of the Model}

In [13], it has been shown that all the experiments with the cork bed material were conducted under dilute transport flow conditions. The dilute transport regime is usually a final stage of fluidization at which very high air creates a very dilute suspension of solids and gas that travel upward together within the riser and there is no backward flow of solids at all. It is characterized by a riser in which an axial voidage distribution is flat except for the profile in the acceleration zone near the solids inlet. The voidage is high and slip velocities approach the terminal velocities of separate particles. At the NETL facility, this regime was obtained at constant superficial velocity (i.e., riser aeration) while the solids mass flux was changed. The solids circulation rate was varied by controlling the aeration at the base of the standpipe. It is obvious from Table (4.1), the riser aeration was kept fixed at $5.25 \mathrm{~m} / \mathrm{s}$ in all the experiments, and the mass flow rate was changed according to the amplitude and period of move air flow.

Experiments representing six different combinations of riser aeration, periods of move air flow and inventory of solids within the CFCFB were used in the analysis; five of these conditions (K39, K41, K36, $\mathrm{K} 35, \mathrm{~K} 37$ and $\mathrm{K} 38$ ) are the replicates of the experiments $\mathrm{K} 30, \mathrm{~K} 31, \mathrm{~K} 32, \mathrm{~K} 33$ and $\mathrm{K} 34$ (out of which the experiments K35 and K37 are the duplicates of the experiment K33). The remaining K40 experiment is of different nature as the period of move air flow is lower (60 seconds). The validation data set consists of 3600 data samples from K39, K41, K36, K35, K37 and K38 experiments, each yielding 600 data samples. The most important aspect during the model development phase is the identification of a one-step-ahead predictor as the new model design is heavily dependent on the accuracy of the $\hat{A}, \hat{B}, \hat{C}$ and $\hat{K}$ matrices. Moreover, it is possible to obtain the estimated SCR directly from Eq. (4.5). In such a situation, the 
interpretation of the state $x(k)$ becomes a fictitious vector as stated in Section 3 that might be considered as some intermediate vector that connects the inputs to the outputs of the CFCFB. The modelfits between the estimated SCR obtained from Eq. (4.5) and those from the combined stochastic SCR and deterministic estimate are compared in Table (4.1). In all these experiments, the combined estimate of SCR is better than those obtained from the one-step-ahead predictor (4.5). This is due to the balanced realization of a new reduced order grey box model where those state vectors were discarded that did not contribute much to the controllability and observability modes of the original system (4.4).

Validation data set K39 has been used for the separate analysis of deterministic and stochastic components. Analysis for other case studies can be similarly performed. The overall prediction result for K39 experiment is illustrated in Fig. (3.1). The dotted red line represents the estimated values while the solid black line indicates the measured values. From this plot, it can be concluded that the prediction of solids circulation rate as the response of combined systems (3.14) and (3.15) is satisfactory.

\begin{tabular}{|c|c|c|c|c|c|c|c|}
\hline $\begin{array}{c}\text { S. } \\
\text { No. }\end{array}$ & $\begin{array}{c}\text { Experiment } \\
\text { No. }\end{array}$ & $\begin{array}{c}\text { Riser } \\
\text { Aeration } \\
(\mathbf{m} / \mathbf{s})\end{array}$ & $\begin{array}{c}\text { Loop Seal } \\
\text { Fluidization } \\
\text { Air }(\mathbf{m} / \mathbf{s})\end{array}$ & $\begin{array}{c}\text { Move Air } \\
\text { Flow }(\boldsymbol{m} / \mathbf{s})\end{array}$ & $\begin{array}{c}\text { Period of } \\
\text { Move Air } \\
\text { Flow (s) }\end{array}$ & A \% & B \% \\
\hline 1. & K36 & 5.25 & 0.25 & 0.15 & 90 & 73.77 & 76.11 \\
\hline 2. & K37 & 5.25 & 0.25 & 0.25 & 120 & 61.24 & 63.98 \\
\hline 3. & K38 & 5.25 & 0.25 & 0.15 & 120 & 74.22 & 76.55 \\
\hline 4. & K39 & 5.25 & 0.25 & 0.25 & 90 & 61.36 & 66.81 \\
\hline 5. & K40 & 5.25 & 0.25 & 0.25 & 60 & 58.63 & 63.32 \\
\hline 6. & K41 & 5.25 & 0.25 & 0.15 & 60 & 74.15 & 75.10 \\
\hline
\end{tabular}

Table (4.1): Comparison of the modelfits between the estimated solids circulation rate obtained from Eq. (4.5) and those from the combined stochastic and deterministic solids circulation rate from the grey box model.

A: Modelfit between measured and estimated SCR obtained from a one-step-ahead predictor Eq. (4.5) in $\%$.

B: Modelfit between measured and estimated SCR obtained from the combined stochastic and deterministic SCR from the grey box model Eqs..(4.14) and (4.15), in \%.

*: Identification data set.

The grey box model parameters for the deterministic and stochastic subsystems of the CFCFB are given below (rounded off to four decimal place):

Deterministic Model:

$$
A_{\text {new }}^{d}=1.0 e+002 *\left[\begin{array}{cccccc}
0.1575 & -0.05764 & -0.0669 & -0.0766 & 0.0069 & -0.0106 \\
0.0080 & -0.0001 & -0.0062 & 0.0014 & -0.0027 & -0.0008 \\
-0.0056 & 0.0033 & 0.0116 & 0.0035 & 0.0049 & 0.0012 \\
0.1195 & -0.0433 & -0.0528 & -0.0562 & 0.0045 & -0.0076 \\
-0.1571 & 0.0636 & 0.0690 & 0.0773 & -0.0055 & 0.0113 \\
1.1418 & -0.4402 & -0.5093 & -0.5992 & 0.0414 & -0.0833
\end{array}\right]
$$




$$
\begin{aligned}
B_{\text {new }}^{d} & =\left[\begin{array}{ccc}
-0.0889 & 0.1114 & -0.1170 \\
0.0244 & 0.0095 & -0.0078 \\
-0.1666 & -0.0166 & 0.0234 \\
-0.0121 & 0.0968 & -0.0994 \\
0.5264 & -0.0956 & 0.1183 \\
-0.0130 & 0.7823 & -0.8745
\end{array}\right] \\
\hat{D}_{\ell x m}^{d} & =\left[\begin{array}{ccc}
0.0002 & -0.0017 & 0.0019 \\
-0.0005 & 0.0038 & -0.0043 \\
-0.0028 & 0.0200 & -0.0224 \\
0.0015 & -0.0105 & 0.0117 \\
0.0004 & -0.0026 & 0.0029 \\
-0.0005 & 0.0034 & -0.0038
\end{array}\right]
\end{aligned}
$$

Stochastic Model:

$$
\begin{aligned}
A_{\text {new }}^{s}= & =\left[\begin{array}{cccccc}
-0.4725 & 0.3455 & 0.4764 & 1.0481 & 0.2884 & 0.2008 \\
-1.5590 & 1.0288 & 0.4890 & 1.2586 & -0.3448 & 0.1530 \\
1.8685 & -0.0569 & 0.2484 & -1.4338 & 0.5993 & 0.0580 \\
-0.8784 & 0.2166 & 0.2807 & 1.5519 & 0.1353 & 0.1580 \\
1.0320 & -0.6138 & -0.6292 & -1.0156 & -0.0622 & -0.2625 \\
-7.0274 & 1.8638 & 2.6158 & 5.0422 & 1.2967 & 1.0949
\end{array}\right] \\
K_{\text {new }}^{s}= & {\left[\begin{array}{ccccccc}
0.7601 & -0.3404 & -0.5079 & -1.0261 & -0.3346 \\
1.8508 & -0.8820 & -0.5570 & -1.0706 & 0.3370 \\
-1.6716 & -0.0544 & -0.1326 & 1.0452 & -0.5362 \\
1.1065 & -0.2327 & -0.3362 & -1.4652 & -0.1884 \\
-1.1629 & 0.2257 & 0.5941 & 1.1627 & 0.1266 \\
6.8215 & -1.9502 & -2.5756 & -5.1383 & -1.526
\end{array}\right] } \\
\hat{D}_{\ell \times(\ell-1)}^{s} & =\left[\begin{array}{ccccccc}
0.9987 & 0.0005 & 0.0001 & -0.0000 & -0.0045 \\
-0.0023 & 1.0008 & 0.0002 & -0.0000 & -0.0080 \\
0.0031 & -0.0011 & 1.0000 & 0.0001 & 0.0106 \\
0.0006 & -0.0002 & -0.0000 & 1.0000 & 0.0020 \\
0.0078 & -0.0027 & -0.0006 & 0.0002 & 1.0268 \\
0.0056 & -0.0020 & -0.0004 & 0.0001 & 0.0193
\end{array}\right]
\end{aligned}
$$




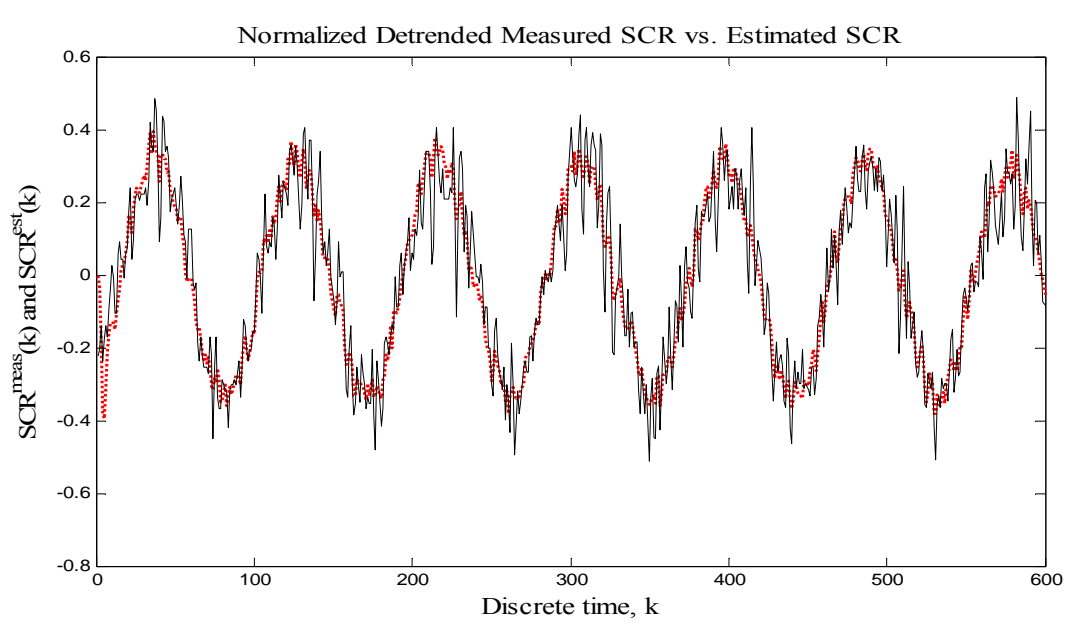

Figure (4.1): Experimental condition - K39. Modelfit between measured and estimated SCR is $66.81 \%$.

\section{5.b Deterministic Analysis of Solids Circulation Rate}

The absolute deviation of the estimated deterministic SCR from the measured one, that is, mean square error (MSE) is calculated as in Eq. (4.19) and it is found to be $4.33 \times 10^{-4}$. The absolute difference between measured and deterministic components (indicated by a solid blue line) is depicted along with both SCR, in Fig. (4.2).

$M S E=\operatorname{var}\left(y_{\text {meas }}(k)-\hat{y}(k)\right)$

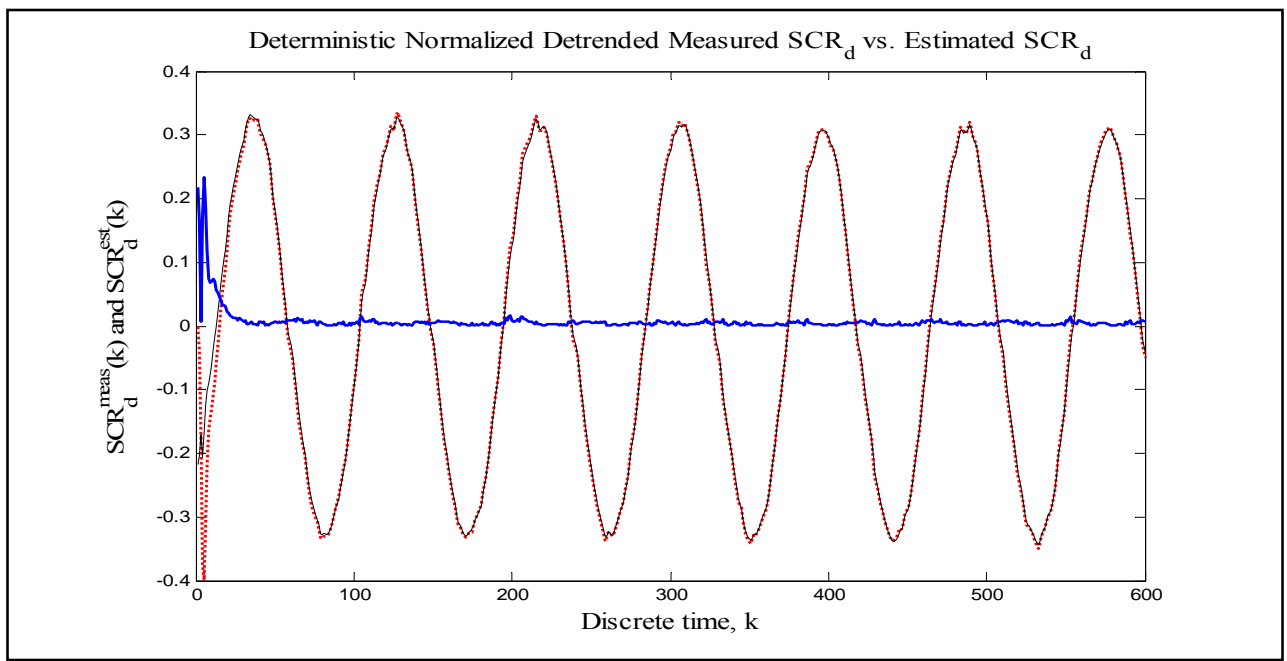

Figure (4.2): Experimental condition - K39. MSE is $\mathbf{4 . 3 3} \times \mathbf{1 0}^{-4}$. Comparison of deterministic measured and estimated solids circulation rate.

As it is known, the standpipe is divided into two portions, the free-fall portion where the solids fall from the bottom of the primary cyclone to the top of the packed bed, and the second packed-bed portion where the solids move much more slowly and as a packed bed. The free-fall portion is a relatively dilute region 
with high velocity and the packed-bed portion is a dense region with low velocity. The top of the packed bed separates these two regions in the standpipe.

It is to be noted that the pressure is greater at the bottom of the riser and standpipe and the pressure difference between the top and bottom generates a gas flow upward (with respect to the standpipe bed). The gas flow can be reasonably predicted using the Ergun equation which predicts pressure drop per length through a packed bed as a function of gas velocity, gas viscosity, bed particle diameter, and bed void fraction. The point to notice here is that the equation is for the pressure drop per length. Stated differently, the slope of a pressure vs height curve is constant and is a function of the above quantities. Using this perspective, it is not surprising that the cork data shows a linear $\Delta P$ vs height $(L)$ graph.

Now that it is understood why a linear graph might be expected, it is discussed about how a non-linear graph might be generated. One of the simplest ways is to have large lengths or high pressure drops. Under these conditions, there might be experienced a large change in pressure from one end of the packed bed to the other. This large pressure change results in a large change in the gas velocity from one end of the bed to the other. And, as stated earlier, the pressure drop is a strong function of gas velocity. Therefore, the $\Delta P / L$ at one point in the bed might be measurably different than the $\Delta P / L$ in another part of the bed. The cork operations did not generate much pressure drop across the bed, however, and hence large velocity differences probably did not occur.

A second method by which the graph might not be linear comes about when it is realized that the solids in the standpipe are aerated. That is to say that additional air is injected into the bed at various locations to assist in the movement of the material. Most of this aeration occurs near the bottom of the bed but there is measurable air used throughout the height of the packed bed portion of the stand pipe. Notice that aeration above the packed bed portion of the stand pipe serves no purpose and hence, is not used. This aeration has the effect of changing the velocity of gas through different portions of the standpipe. Different velocities result in different $\Delta P / L$. Again, it is believed that these aeration flows were relatively small in the present case and hence, did not result in much of a change in $\Delta P$ vs $L$ curve slope. 


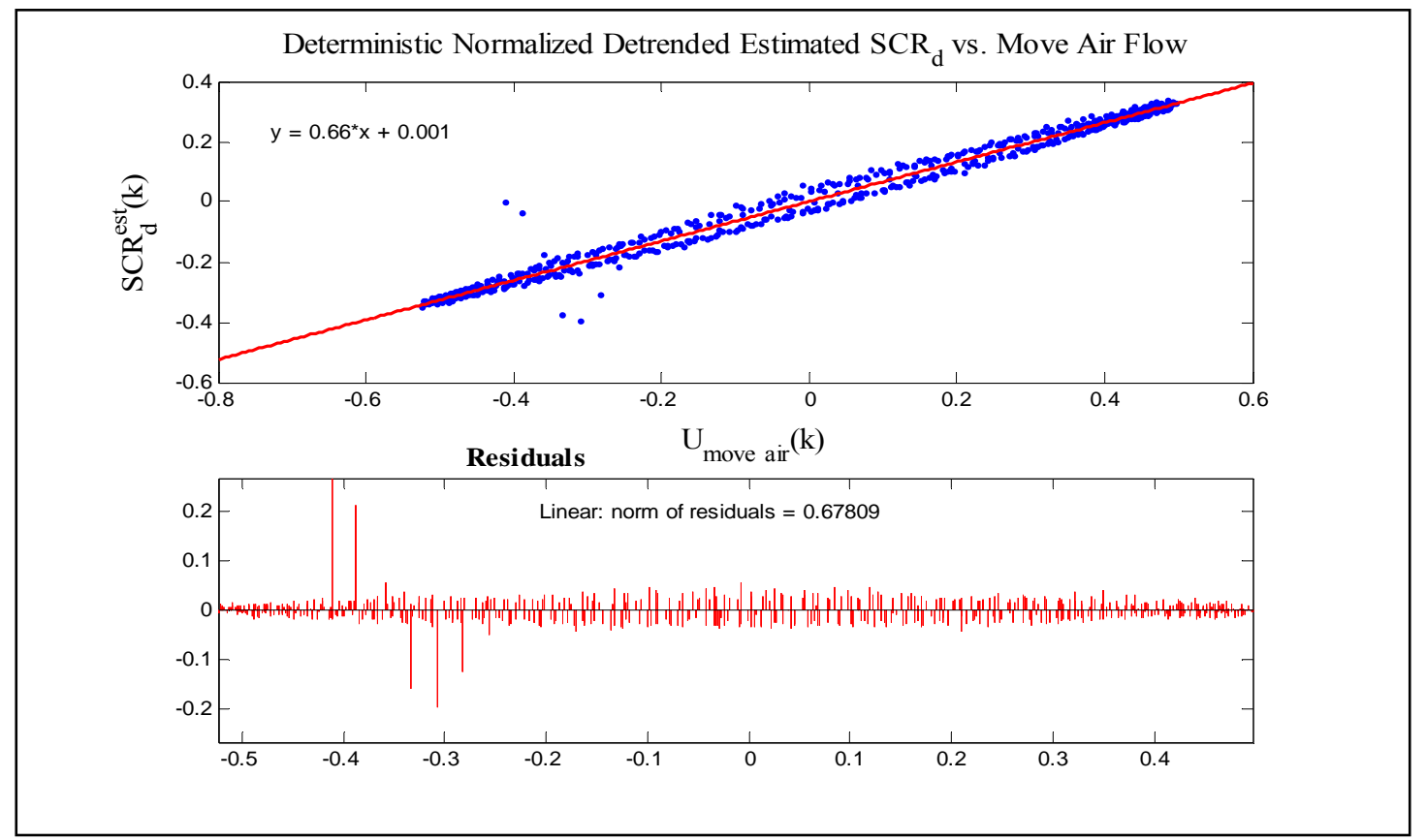

Figure (4.3): Experimental condition - K39. Comparison of deterministic estimated solids circulation rate and move air flow to verify linear relationship between them.

Above observation leads to the conclusion that the pressure profile is a linear function of height under present conditions of move air flow and other aerations across the stand pipe. This advocates if there also exists any linear relationship between solids circulation rate and move air flow under these operating conditions. To investigate this relationship, a plot between deterministic solids circulation rate and move air flow is plotted in Fig. (4.3). A significant linear relationship between the deterministic SCR and move air flow is observed. There are few outliers in the move air and SCR plot that has degraded the residual calculation. They arise due to initial values of SCR during estimation. When the outliers are removed, the norm of residual becomes 0.5067 . The absolute deviation of linear fit to those of higher order polynomials (quadratic to $10^{\text {th }}$ degree polynomial) lies within $0.05 \%-0.13 \%$. Thus, the deterministic model derived in this work independently shows that the solids circulation rate responds linearly to the move air flow without any relevance of a pressure profile being linearly dependent upon the height in the dense region. This linearity further affirms the correct initial assumption for a CFCFB that it acts as a linear system particularly in a packed bed portion of the stand pipe when the riser operates under dilute transport condition with the cork bed material.

\section{5.c Stochastic Analysis of Solids Circulation Rate}

In the K39 case as well, the absolute deviation of the estimated stochastic SCR from the measured one is calculated in terms of Eq. (4.19) and it is found to be 0.0055. A plot of the absolute difference between measured and stochastic components (indicated by a solid blue line) is depicted in Fig. (4.4). 


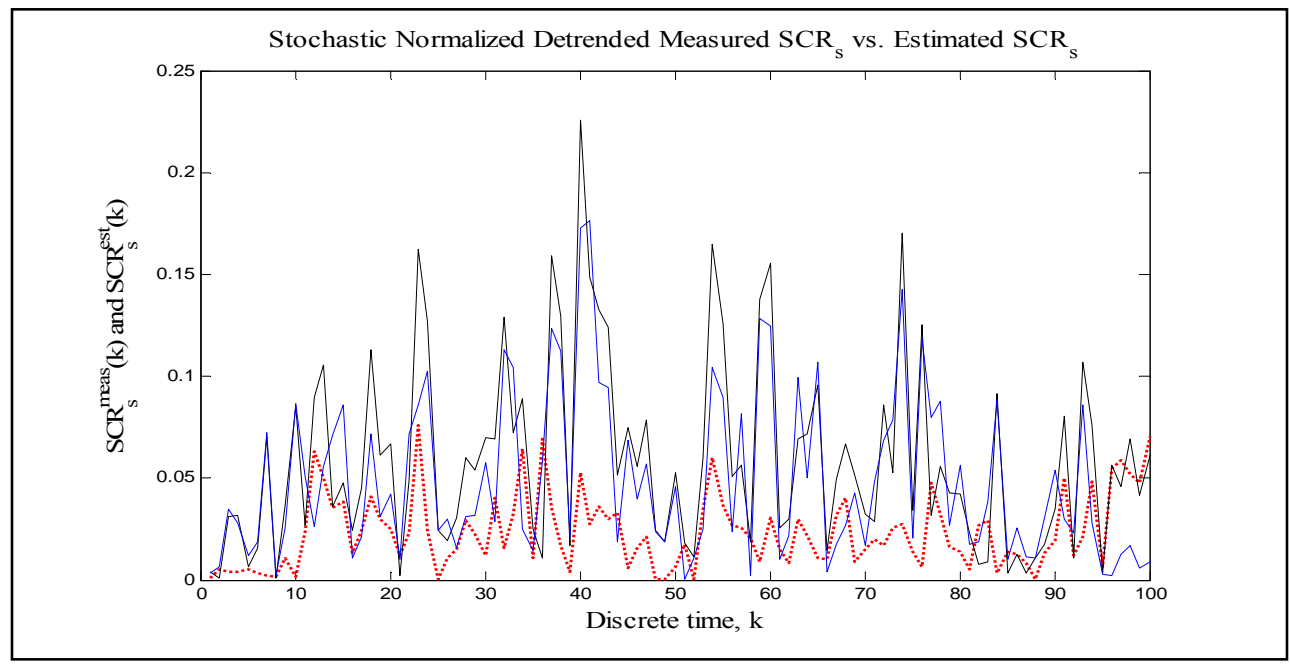

Figure (4.4): Experimental condition - K39. MSE is 0.0055. Comparison of stochastic estimated solids circulation rate and measured SCR. 100 samples of absolute values are plotted.

The next step is to test whether the samples from stochastic solids circulation rate follow a normal distribution. As each sample is small in magnitude due to normalization of the original samples, Lilliefors test was considered to be a good choice to perform the check. The result of hypothesis test is 1 if the hypothesis can be rejected that the input data has normal distribution or 0 if the hypothesis cannot be rejected. The hypothesis can be rejected if the test is significant at the $5 \%$ interval. This test compares the empirical distribution of the input data with a normal distribution having the same mean and variance as stochastic solids circulation rate.

With the present validation data set, the Lilliefors test statistics of 0.024 and 0.026 are obtained for measured stochastic SCR and estimated stochastic SCR, respectively. These values are larger than the cutoff value of 0.036 for a $5 \%$ level test but fall outside the range of the Lilliefors table. However, as the result of the hypothesis test indicates whether to reject the hypothesis, it came out to be 0 for both the inputs in the present case. Hence, the hypothesis that both the stochastic SCR has normal distribution is not rejected.

To visualize the distribution, a histogram can be made (Figs.(4.5) and (4.6)) or the probability distribution function can be calculated using a kernel smoothing method Bowman[42] and plotted (Fig.(4.7)). These graphs show that the distribution for measured and estimated stochastic solids circulation rate with mean andvariance, $\bar{y}_{S C R}^{s}=-0.0032, \operatorname{Var}\left(y_{S C R}^{s}\right)=0.0081$ and $\hat{\bar{y}}_{S C R}^{s}=-0.00088, \operatorname{Var}\left(\hat{\bar{y}}_{S C R}^{s}\right)=0.00094$, respectively are similar to that of a normal distribution curve but the graphs for estimated stochastic solids circulation rate is slightly skewed to the right. This might be the case because the stochastic estimates of SCR is determined from the pressure drops across the unit, and except loop seal pressure drop, all other pressure drops lead the solids circulation rate as depicted in Fig. (4.8). 


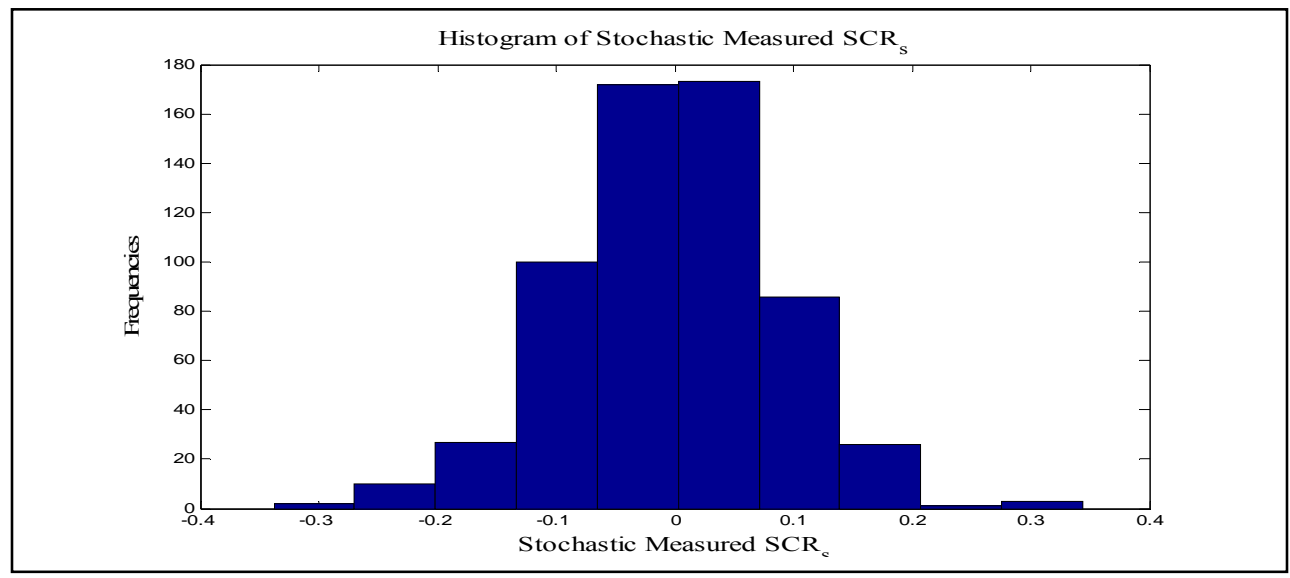

Figure (4.5): Experimental condition - K39. Histogram for stochastic measured solids circulation rate.

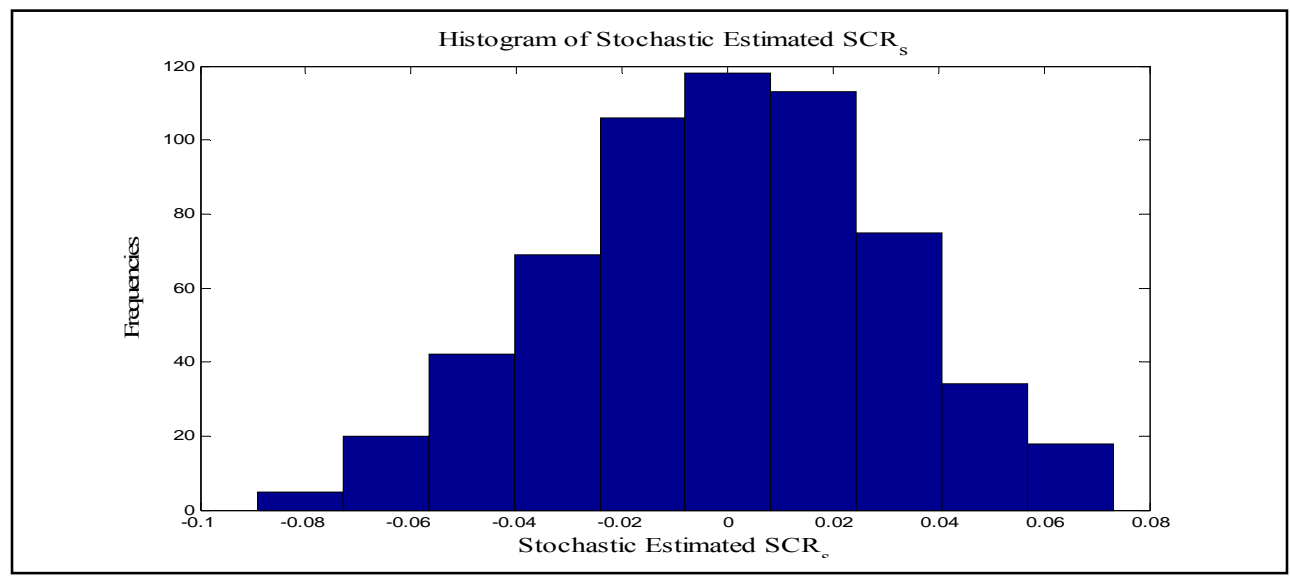

Figure (4.6): Experimental condition - K39. Histogram for stochastic estimated solids circulation rate.

To further investigate whether both the measured and estimated stochastic SCR indeed follow a normal distribution, normal probability plots have been shown in Figs. (4.9) and (4.10). Superimposed on the plots is a line (indicated by a red line) joining the first and third quartiles of each column of input data (a robust linear fit of the sample order statistics). This line is extrapolated out to the ends of the sample to help evaluate the linearity of the data. If the data does come from a normal distribution, the plot will appear linear which exactly is the case with our present SCR. Other probability density functions will introduce curvature in the plot. The conclusion that can be derived from this observation is that, the measurements from the pressure taps and the spiral give the measurement errors that follow a Gaussian distribution. 


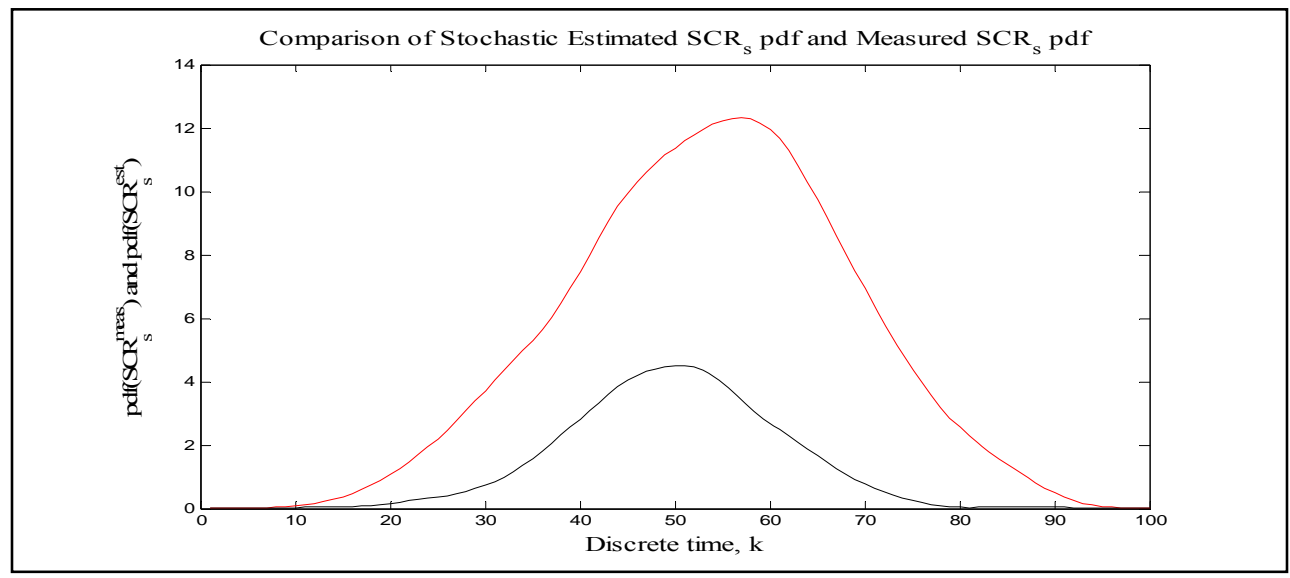

Figure (4.7): Experimental condition - K39. Comparison of probability density function of stochastic measured and estimated solids circulation rate.

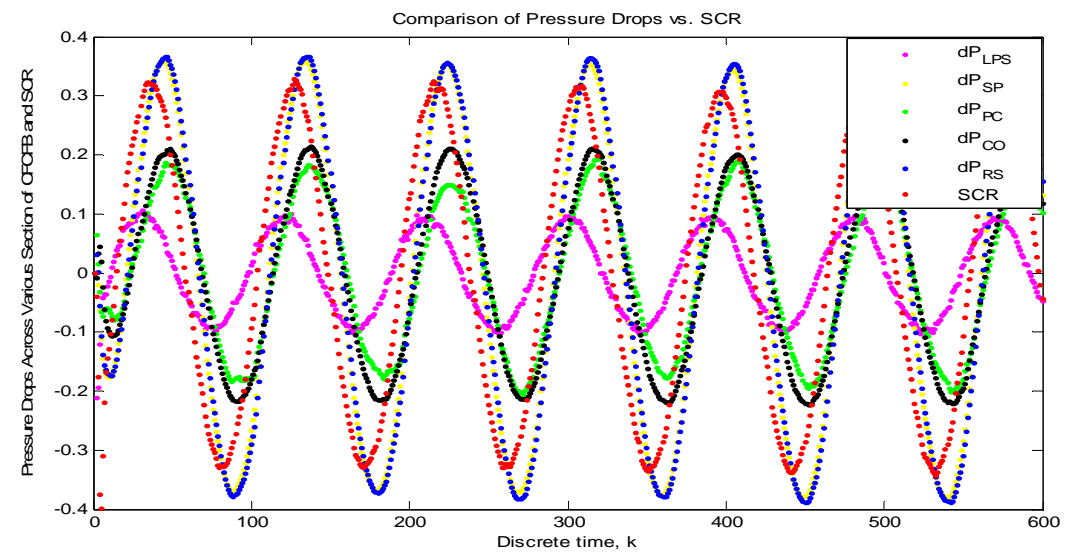

Figure (4.8): Experimental condition - K39. Plot of pressure drops across various sections of the CFCFB vs. deterministic solids circulation rate to indicate all pressure drops lead SCR except $\triangle P_{L P S}$.

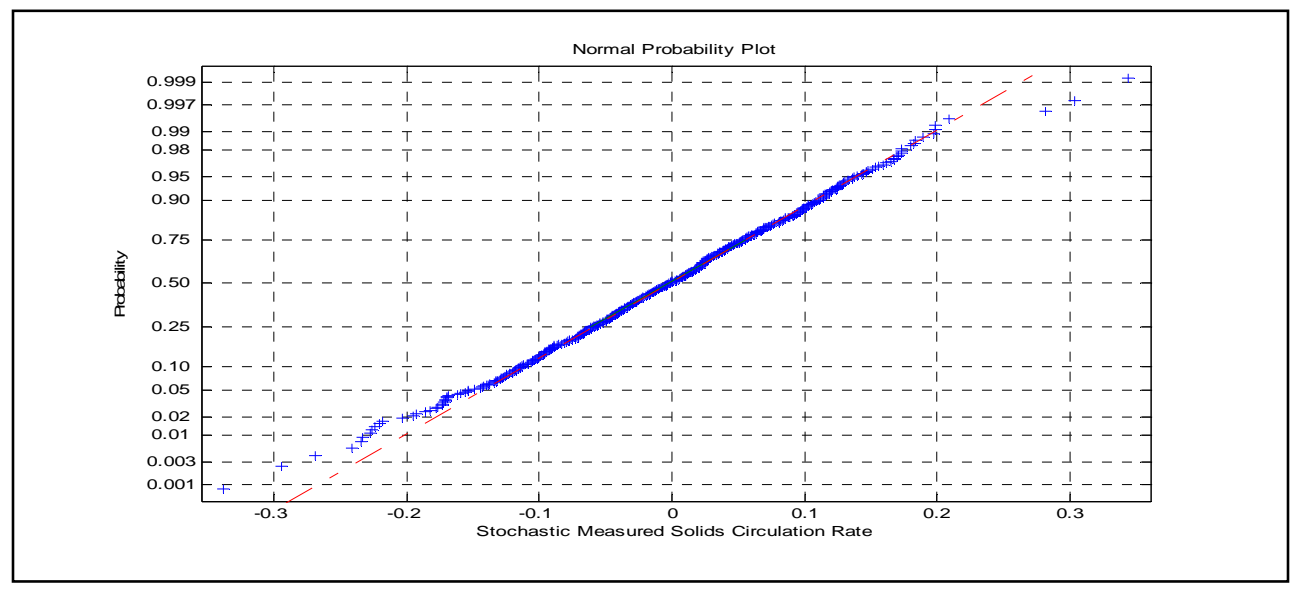

Figure (4.9): Experimental condition - K39. Normality plot to show stochastic measured solids circulation rate indeed follows a normal distribution. 


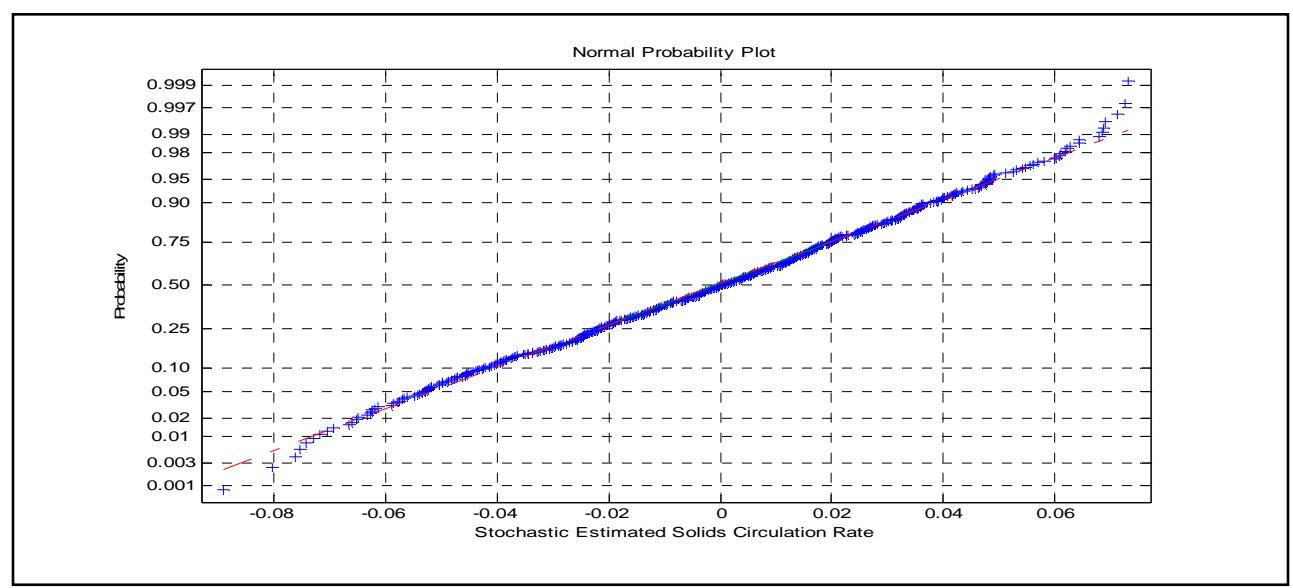

Figure (4.10): Experimental condition - K39. Normality plot to show stochastic estimated solids circulation rate indeed follows a normal distribution.

Thus, a generalized theory has been developed that converts a complete black box model obtained from prediction error method, to a grey box model which does not include any fictitious variable without physical meaning within the given system. Hence, the interaction between physical variables could be interpreted. During this model development, both the cases are considered including when the order of the black box model is equal to or is greater than the number of output measurements. Separate analysis of solids circulation rate has been done. The linear relationship between the solids circulation rate and a move air flow has been rationalized with the use of deterministic component. The measurement error that persists in the pressure tap measurements and spiral measurements during operation, are justified to follow a normal distribution using stochastic counterpart. The combined estimate of the stochastic and deterministic SCR that is obtained with only three independent aerations inputs in the CFCFB such as move air flow, riser aeration and loop seal fluidization air over different operating conditions achieved using cork as the bed material during the experiments is compared in terms of the modelfits with that of the solids circulation rate measurement from the physical unit. Finally, experimental result has been shown for deterministic, stochastic and combined estimates of solids circulation rate.

\subsection{Procedure}

i. Choose inputs and outputs of the system under consideration according to some physical insight about the system and with the application in mind. Select a proper model structure (4.4).

ii. Extracting information from data is not an entirely straightforward task. In addition to the decisions required for model structure selection and validation, the data might need to be handled carefully. If physical levels do not play a role in the selected model, then detrend the data by removing their mean values. The models will then describe how changes in the input give changes in output, but not explain the actual levels of the signals. This is the normal situation.

iii. Determine system matrices $\hat{A}, \hat{B}, \hat{K}$, and $\hat{C}$ using PEM if the data were collected under closed loop or use N4SID if they were collected under open loop. Fine tune the order of a black box model such that the cross correlation function between residuals and input does not go significantly outside the confidence region. Otherwise there is something in the residuals that originate from the input, and has not been properly taken care of by the model.

iv. Obtain a model as in Eq. (4.9) to determine innovation term for the variable of interest from other output measurements used in the PEM.

v. If $n=\ell$, use output-to-state transformation to arrive at a model in Eq. (4.10). 
vi. If $n>\ell$, proceed as follows: First, start with the balanced realization of the black box system, reduce the model order until $n=\ell$ on the basis of smaller values of $g(i)$. Then, through the output-to-state transformation, obtain grey box models (4.14) and (4.15).

vii. Compute model fit between estimated and measured output according to Eq.(4.16) to test if the model derived has captured the desired dynamics of the system.

viii. Do the separate analysis of deterministic and stochastic component of a physical variable chosen.

A final point to address is that if the user is also interested in estimating various pressure drops across the CFCFB in the same way as has been done for the solids circulation rate, the same procedure can be adopted. First, the original measurements of the pressure drops and the SCR should be employed to determine the one-step-ahead predictor and then to determine the noise and deterministic sub models and the new grey box model through output-to-state transformation assuming that these pressure drops are not measurable. However, one of the pressure drops across the CFCFB should at least assumed to be measurable such that the innovation term for this pressure drop can be derived from its original measurements and the innovation term for the other pressure drops and SCR can be deduced accordingly.

\subsection{Limitation of Present Approach}

The experimental conditions with the cork material exhibit a linear relationship between the move air flow and solids circulation rate. For operations where the relationship between these two variables is nonlinear such as expected when operating the CFCFB in the fast fluidized regime, the present method may not be able to give a desired result as in the present case. In such a situation a multiple model identification technique as in Chapter 3 could be employed during identification to determine CFB parameters and a conversion to the grey box model can be similarly implemented. 


\section{Chapter 5}

\section{Air Flow Split and Route Estimation in a Cold Flow Circulating Fluidized Bed}

\subsection{Motivation}

According to the discussion made in Chapter 1, it can be concluded that move air flow, aeration injected into the standpipe just above the entrance of the non-mechanical valve, severely affects the dependent variables that are interrelated to each other within the cold flow circulating fluidized bed and is the most important parameter for successful operation of the given system. During the CFCFB process, the split of a move air flow takes place between the standpipe and the non-mechanical valve connecting the riser and the standpipe as shown in Fig. (5.1). This split is not known during operation and hence, it has to be assumed in the modeling efforts and other applications, if required. Such an assumption is employed in Jalali et. al. [15], Shim et. al. [16], while simulating the one-dimensional-dynamic-model of the standpipe.
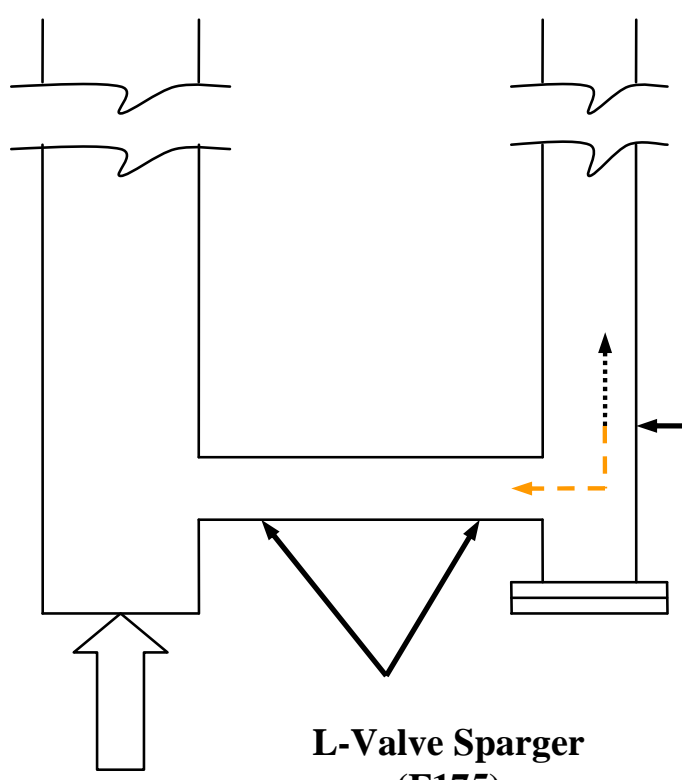

\section{Riser Aeration}

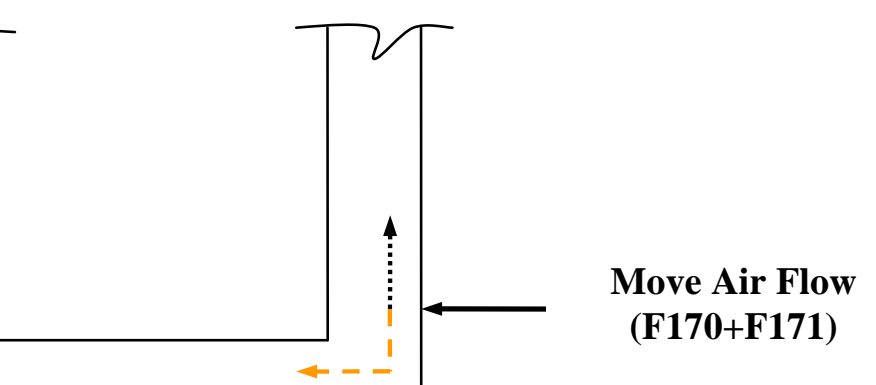

(F431)

\section{…... Move air flow split between the standpipe and L-valve}

Figure (5.1): Illustration of a split of move air flow between the standpipe and L-valve of a cold flow circulating fluidized bed.

Alternatively, the additional aerations, injected into the packed bed at various locations of the standpipe, act as the air bias to fluidize the standpipe column. It is very common that at some point, these aeration flows split with some of the flows moving upward and some moving downward. A similar analysis would 
then be considering the aeration flows (split) which are not the move air within the standpipe. Therefore, the motivation reasonably includes estimating the route within the standpipe that each aeration follows during operation.

On the other hand, the non-mechanical comprising of an L-valve that allows the solids to move from the standpipe to the riser across the pressure barrier, does not allow the gas to move from the riser to the standpipe. Therefore, it is not necessary to estimate the air flow split between the riser and the L-valve.

As the pressure drops are the most commonly measured parameters in studies of fluidized beds Brown et. al. [43], especially in identifying different fluidization regimes within the circulating fluidized bed risers Johnsson et. al. [44], Zijerveld et. al. [45], Alberto et. al. [46], a novel method is developed in the present chapter utilizing the pressure drops across the two bottom portions of the CFCFB to determine and assert the split of a move air flow between the standpipe and the L-valve. This split is estimated describing a pressure drop at the bottom of the standpipe at the current time as a function of the bottom pressure drop and a product of average mass flux and move air flow split at previous time instant and using a recursive prediction error method on this real time model to determine the model parameters. The estimate is then verified assuming the pressure drop across the L-valve is a function of a split of move air and the L-valve aeration and comparing the measured L-valve pressure drop with those of estimated values.

To estimate the path of additional aeration flows within the standpipe, a similar procedure is designed. The design technique is based on the relative velocity between the gas and solids velocities. The split parameter for each aeration including the move air is chosen per second as a slip velocity divided by a height of the desired location. During simulation, they are then kept within the range from -1 to 1 , where 1 indicates that whole aeration flows in the downward direction and vice versa. The solids superficial velocity required is obtained from the measured solids circulation rate and average void fraction in a moving bed condition and lean phase void fraction chosen by a user under a fluidization condition, and it is assumed to be same throughout the standpipe, Huang [13].

\subsection{Pressure Drop across the Bottom of the Standpipe}

The pressure drop at the bottom the standpipe is related to the gas void fraction and relative gas and solids velocities according to Ergun [47], which is an excellent correlation over a wide range of voidage from 0.36 to 0.92 . The modified Ergun equation, [13] is presented below:

$$
\frac{\Delta P_{\text {bottom }, S P}}{L_{\text {bottom }, S P}}=\frac{150 \mu}{\left(\xi d_{s v}\right)^{2}} \frac{(1-\varepsilon)^{2}}{\varepsilon^{2}} U_{\text {slip }}+\frac{1.75 \rho_{p}}{\xi d_{s v}} \frac{(1-\varepsilon)}{\varepsilon}\left|U_{\text {slip }}\right| U_{\text {slip }}
$$

It is assumed that solids always move faster than gas such that gas flows upwards with respect to the downward flowing solids; therefore, the slip velocity defined as in Knowlton et. al. [48] becomes:

$$
U_{\text {slip }}=\frac{U_{s}}{1-\varepsilon}-\frac{U_{g}}{\varepsilon}=\frac{U_{m f}}{\varepsilon_{m f}} \frac{\left(\varepsilon-\varepsilon_{p b}\right)}{\left(\varepsilon_{m f}-\varepsilon_{p b}\right)}
$$

Defining $C_{1}=\frac{150 \mu}{\left(\xi d_{s v}\right)^{2}}$ and $C_{2}=\frac{1.75 \rho_{p}}{\xi d_{s v}}$ and differentiating Eq. (5.1) with respect to time under the assumption that $\varepsilon((x, y, z), t)=\varepsilon((0), t)$ at the dense phase of the standpipe, we obtain 
$\frac{d \Delta P_{\text {bottom }, S P}(t)}{d t}=a(t) \Delta P_{\text {bottom }, S P}(t)+X^{*} L_{\text {bottom }, S P}$

Where,

$a(t)=\frac{d \log _{e} U_{\text {slip }}(t)}{d t}$

and

$X=\left[C_{1}\left(\frac{2}{\varepsilon^{2}}-\frac{2}{\varepsilon^{3}}\right) \frac{d \varepsilon}{d t}-C_{2} \frac{1}{\varepsilon^{2}} \frac{d \varepsilon}{d t}+C_{2} \frac{(1-\varepsilon)}{\varepsilon} \frac{d U_{\text {slip }}}{d t}\right] U_{\text {slip }}$

Multiplying $X$ by $L_{b o t t o m, S P}$ and manipulating Eq. (5.5), we obtain

$X^{*} L_{\text {bottom }, S P}=b_{t} U_{g}$

Where

$b_{t}=\left[C_{1}\left(\frac{2}{\varepsilon^{2}}-\frac{2}{\varepsilon^{3}}\right) \frac{d \varepsilon}{d t}-C_{2} \frac{1}{\varepsilon^{2}} \frac{d \varepsilon}{d t}+C_{2} \frac{(1-\varepsilon)}{\varepsilon} \frac{d U_{\text {slip }}}{d t}\right] *\left(\frac{U_{s}}{U_{g}(1-\varepsilon)}-\frac{1}{\varepsilon}\right) * L_{\text {bottom }, S P}$

Therefore, the pressure drop dynamics at the bottom of the standpipe can be represented by the following equation:

$\frac{d \Delta P_{\text {bottom }, S P}(t)}{d t}=a(t) \Delta P_{\text {bottom }, S P}(t)+b_{t} U_{g}(t)$

Hence, the parameters $a(t)$ and $b_{t}$ need to be determined to simulate Eq. (5.8). The basic idea would be to estimate them from the observed input-output data of a given system. It is to be noted that the unit of $b_{t}$ is $\mathrm{Kg} /\left(\mathrm{m}^{2} \cdot \mathrm{s}^{2}\right)$. Let us decompose the parameter $b_{t}$ into two components as follows:

$b_{t}=b_{1}(t) * b_{2}(t)\left(=K g /\left(m^{2} . s\right) * 1 / s\right)$

where $b_{1}(t)$ has the unit of mass flux and $b_{2}(t)$ represents the move air flow split per second.

Eq. (5.7) is too difficult to solve analytically to find the explicit form for $b_{l}(t)$. Perhaps, the better way to deal with this problem is to assume $b_{l}(t)$ as the average mass flux at the bottom of the standpipe i.e., through continuity consideration, the voidage $\varepsilon$ can be expressed as:

$\varepsilon=1-\frac{G_{s}}{\rho_{p} U_{s}}$ 
and assuming $b_{1}(t)=\left.G_{S, a v}\right|_{\varepsilon=\varepsilon_{a v}=\frac{\varepsilon_{m f}+\varepsilon_{p b}}{2}}$, we obtain using Eqs. (5.2) and (5.10),

$b_{l}(t)=\rho_{p}\left(1-\varepsilon_{a v}\right)^{2}\left(\frac{U_{g}(t)}{\varepsilon_{a v}}+\frac{U_{m f}}{\varepsilon_{m f}}\left(\varepsilon_{a v}-\varepsilon_{p b}\right)\right)$

The bottom standpipe pressure drop measurement is available at the NETL test facility and the move air flow can be used to find gas velocity. However, the measurements at NETL are recorded at $1 \mathrm{~Hz}$ for the present set of data; therefore, Eq. (5.8) is required to be discretized. The measurement of pressure drop is contaminated by noise and it should be taken into consideration. Furthermore, there will always be a split of move air flow between the standpipe and the L-valve and the parameter $b_{2}(t)$ is discretized to $b_{s p l i t}^{l}(k)$ to identify this split. The discretized equation then becomes:

$$
\Delta P_{\text {bottom }, S P}(k)=a_{1}(k-1) \Delta P_{\text {bottom }, S P}(k-1)+\Delta k b_{1}(k-1) b_{\text {split }}^{l}(k-1) U_{\text {MoveAir }}(k-1)+e_{1}(k)
$$

Eq. (5.12) states that the pressure drop at the bottom of the stand pipe, $\Delta P_{b o t t o m, S P}$, at discrete time instant $k$ is a linear function of the bottom pressure drop and the product of average mass flux $\left(b_{1}(k-1)\right)$ and split of move air flow $U_{\text {Moveair }}$ at previous time instant $k-1$. The variable $e_{1}(k)$ is assumed to be a white noise source with covariance matrix $E e_{1}(k) e_{1}(k)^{T}=R_{1}$ in the pressure drop measurements. The model parameters $a_{1}(k-1)=1+\Delta k * a(k-1)$ at $\Delta k$ seconds, and $b_{\text {split }}^{l}(k-1)=b_{2}(k-1)$ per second where $a(k-1)$ and $b(k-1)$ are the discretized parameters of $a(t)$ and $b_{t}$, respectively at previous time instant $k-1$, are to be determined from the previous measurements of the bottom standpipe pressure drop and move air flow.

\subsection{Recursive Prediction Error Method}

The estimate of Eq. (5.12) can be written as

$$
\Delta \hat{P}_{\text {bottom }, S P}(k)=\hat{a}_{l}(k-1) \Delta \hat{P}_{\text {bottom }, S P}(k-1)+\hat{b}(k-1) U_{\text {MoveAir }}(k-1)
$$

Eq. (5.13) can also be rewritten as

$$
\Delta \hat{P}_{\text {bottom }, S P}(k)=\varphi^{T}(k) \hat{\theta}(k-1)
$$

where

$$
\begin{aligned}
& \varphi^{T}(k)=\left[\begin{array}{ll}
\Delta P_{\text {bottom }, S P}(k-1) & U_{\text {MoveAir }}(k-1)
\end{array}\right] \\
& \hat{\theta}(k-1)=\left[\begin{array}{ll}
\hat{a}_{1}(k-1) & \hat{b}(k-1)
\end{array}\right]^{T}
\end{aligned}
$$


The most logical approach to the adaptation problem is to assume a certain model for how the "true" parameters $\theta_{\text {true }}$ change, Ljung [25]. A typical choice is to describe these parameters as a random walk.

$\theta_{\text {true }}(k)=\theta_{\text {true }}(k-1)+w(k)$

Here $w(k)$ is assumed to be white Gaussian noise with covariance matrix

$E w(k) w^{T}(k)=R_{2}$

A typical recursive algorithm is then

$\hat{\theta}(k)=\hat{\theta}(k-1)+K(k)\left(\Delta P_{b o t t o m, S P}(k)-\Delta \hat{P}_{b o t t o m, S P}(k)\right)$

The gain $K(k)$ determines in what way the current prediction error $\Delta P_{\text {bottom }, S P}(k)-\Delta \hat{P}_{\text {bottom }, S P}(k)$ affects the update of the parameter estimate. It is typically chosen as

$K(k)=Q(k) \varphi(k)$

where $\varphi(k)$ can also be interpreted as an approximate of the gradient with respect to $\theta$ of $\Delta \hat{P}_{\text {bottom }, S P}(k \mid \theta)$ at $\theta=\hat{\theta}(k-1)$. An optimal choice of $Q(k)$ can be computed from the Kalman filter, and the complete algorithm becomes

$$
\begin{aligned}
& \hat{\theta}(k)=\hat{\theta}(k-1)+K(k)\left(\Delta P_{\text {bottom }, S P}(k)-\Delta \hat{P}_{\text {bottom }, S P}(k)\right) \\
& \Delta \hat{P}_{\text {bottom }, S P}(k)=\varphi^{T}(k) \hat{\theta}(k-1) \\
& K(k)=Q(k) \varphi(k) \\
& Q(k)=\frac{P(k-1)}{\lambda+\varphi^{T}(k) P(k-1) \varphi(k)} \\
& P(k)=\frac{1}{\lambda}\left(P(k-1)-\frac{P(k-1) \varphi(k) \varphi^{T}(k) P(k-1)}{\lambda+\varphi^{T}(k) P(k-1) \varphi(k)}\right)
\end{aligned}
$$

This algorithm is called the forgetting factor approach to adaptation, with the forgetting factor $\lambda$. The algorithm is also known as recursive least squares in the linear regression case. Here, the approach is to discount old measurements exponentially, so that an observation that is $\tau$ samples old carries a weight that is $\lambda^{\tau}$ of the weight of the most recent observation. This means that the following function is minimized at time $k$.

$$
\sum_{\kappa=1}^{k} \lambda^{k-\kappa} e^{2}(\kappa)
$$


Here $\lambda$ is a positive number (slightly) less than 1 . The measurements that are older than $\tau=1 /(1-\lambda)$ carry a weight in the expression above that is less than about 0.3 . Think of $\tau=1 /(1-\lambda)$ as the memory horizon of the approach. Typical values of $\lambda$ are in the range 0.97 to 0.995 .

\subsection{Pressure Drop across the L-valve}

Rather than opting the procedure that is developed to model the standpipe bottom pressure drop, we use a general proposition to obtain the state space equation for determining the pressure drop across the L-valve such that wide range of particles can be accommodated. A discrete time state-space description for the Lvalve pressure drop is described by

$$
\Delta P_{L V}(k+1)=f\left(\Delta P_{L V}(k), U_{g}(k)\right)
$$

where $U_{g}(k)=\left[U_{L V A}(k) U_{\text {MoveAir }}(k)\right]^{T}$. Let the function $f$ be linearized at the operating point $\left(\Delta P_{L V}(o), U_{g}(o)\right)=(0,0)$ using a first-order Taylor series expansion. Then, we obtain

$$
f\left(\Delta P_{L V}(k), U_{g}(k)\right) \approx f\left(\Delta P_{L V}(0), U_{g}(0)\right)+a_{2}(k)\left(\Delta P_{L V}(k)-\Delta P_{L V}(o)\right)+B_{2}(k)\left(U_{g}(k)-U_{g}(o)\right)
$$

with

$$
\begin{aligned}
& f\left(\Delta P_{L V}(o), U_{g}(o)\right)=0 \\
& a_{2}(k)=\frac{\partial f}{\partial \Delta P_{L V}} \mid\left(\Delta P_{L V}, U_{g}\right)=\left(\Delta P_{L V}(k), U_{g}(k)\right) \\
& B_{2}(k)=\frac{\partial f}{\partial U_{g}^{T}} \mid\left(\Delta P_{L V}, U_{g}\right)=\left(\Delta P_{L V}(k), U_{g}(k)\right)
\end{aligned}
$$

Therefore, Eq. (5.22) becomes

$$
\Delta P_{L V}(k+1)=a_{2}(k) \Delta P_{L V}(k)+B_{2}(k) U_{g}(k) \Rightarrow \Delta P_{L V}(k)=a_{2}(k-1) \Delta P_{L V}(k-1)+B_{2}(k-1) U_{g}(k-1)(5.2
$$

Let us assume that the parameter $B_{2}$ consists of the following terms:

$$
B_{2}=\left\lfloor\begin{array}{ll}
b_{L V} & b_{M f} * \bar{b}_{2}
\end{array}\right\rfloor
$$

where $b_{L V}$ and $b_{M f}$ are in $\mathrm{Kg} /\left(\mathrm{m}^{2} . s\right)$ and $\bar{b}_{2}$ is a dimensionless quantity. To determine the split parameter $b_{s p l i t}^{2}$ for the move air flow in the L-valve per second, let us decompose the parameter $\bar{b}_{2}$ into $\bar{b}_{2}=\Delta k * b_{\text {split }}^{2}$ where $\Delta k$ should be same as used in the discretization of Eq. (5.8) such that the split takes place between the standpipe and L-valve at the same time instant. 
Even if the interpretation of model parameters are lost with the above state-space description, when we compare Eq. (5.24) with Eq. (5.12), we find that Eq.(5.24) is similar to the one obtained by using Ergun equation. The difference lies in the gas velocities term, consisting of two components rather than one. It is then reasonable to correlate these gas velocities with the aerations deduced in Yang et. al. [49]. On the other hand, the $a_{2}$ term should not be interpreted as something related to a slip velocity as in Eq. (5.4) because the slip term is usually assumed to be 0 in the L-valve [49] and therefore, the reasonable elucidation for $a_{2}$ might be to consider it as some internal variable inside the L-valve that connects the pressure drop dynamics at present time instant with the previous pressure drop.

The parameter $\hat{b}_{M f}$ may be calculated from the following gas entrainment equation [49] under no slip condition utilizing voidage at minimum fluidization:

$b_{M f}(k)=G_{s L V}=\frac{\rho_{p} A_{S P}}{A_{L V}} \frac{\left(1-\varepsilon_{m f}\right)}{\varepsilon_{m f}} U_{\text {MoveAir }}$

Therefore, the parameter vector $B_{2}$ becomes:

$B_{2}=\left[b_{L V} \quad \Delta k \frac{\rho_{p} A_{S P}}{A_{L V}} \frac{\left(1-\varepsilon_{m f}\right)}{\varepsilon_{m f}} * b_{\text {split }}^{2} * U_{\text {MoveAir }}\right]$

Consequently, the L-valve pressure drop model incorporated with measurement error gives:

$\Delta P_{L V}(k)=a_{2}(k-1) \Delta P_{L V}(k-1)+b_{L V}(k-1) U_{L V A}(k-1)+\Delta k \frac{\rho_{p} A_{S P}}{A_{L V}} \frac{\left(1-\varepsilon_{m f}\right)}{\varepsilon_{m f}} * b_{\text {split }}^{2}(k-l) *\left(U_{\text {MoveAi }}(k-1)\right)^{2}+e_{2}(k)$

Hence, the estimate of the L-valve pressure drop can also be determined using the recursive algorithm (5.20) on the following model:

$\Delta \hat{P}_{L V}(k)=\psi^{T}(k) \hat{\chi}(k-1)$

where

$$
\begin{aligned}
& \psi^{T}(k)=\left[\begin{array}{lll}
\Delta \hat{P}_{L V}(k-1) & U_{L V A}(k-1) & \Delta k \frac{\rho_{p} A_{S P}}{A_{L V}} \frac{\left(1-\varepsilon_{m f}\right)}{\varepsilon_{m f}} *\left(U_{\text {MoveAir }}(k-1)\right)^{2}
\end{array}\right] \\
& \hat{\chi}(k-1)=\left[\begin{array}{lll}
\hat{a}_{2}(k-1) & \hat{b}_{L V}(k-1) & \hat{b}_{\text {split }}^{2}
\end{array}\right]^{T}
\end{aligned}
$$

where $\hat{b}_{\text {split }}^{2}(k-1)=1-\hat{b}_{\text {split }}^{1}(k-1)$ is determined from the move air flow and the pressure drop measurement at the bottom of the standpipe and it is kept fixed during the recursive identification of the L-valve pressure drop. This ensures if the split of a move air flow between the two components is indeed 
correct because the L-valve pressure drop modeled as a function of L-valve aeration and remaining move air flow should track its measured values provided the split of move air is correct.

\subsection{Route Estimation for Aerations Fed Across the Standpipe}

To validate if the split of aerations within the standpipe are correct, we have chosen the location where the spiral vane is placed Ludlow et. al. [20], to model the pressure drop at that location as a function of the split of these aerations, and comparing its estimate with the measured values. With the similar method developed for the pressure drop across the bottom of the standpipe using Ergun equation, we obtain the following discrete time model for the respective pressure drop:

$$
\Delta P_{\text {spiralLocation }}(k)=a_{3}(k-1) \Delta P_{\text {spiralLocation }}(k-1)+\Delta k_{1} B_{3}(k-1) U_{A e r}(k-1)+e_{3}(k)
$$

Where

$$
\begin{aligned}
& B_{3}=b_{3} *\left[\begin{array}{lllll}
b_{11} & b_{12} & b_{13} & b_{14} & b_{15}
\end{array}\right] \\
& U_{A e r}=\left[\begin{array}{lllll}
U_{A} & U_{A 1} & U_{A 2} & U_{A 3} & U_{A 4}
\end{array}\right]
\end{aligned}
$$

where $U_{A}=b_{\text {split }}^{l} * \Delta k * U_{\text {MoveAir }}$. Split of move air flow is to be used as the split took place at the bottom of the standpipe. Then the RPEM can be used to determine model parameters.

When the solids travel down the standpipe with some velocity, they drag some air with them and the absolute value of gas in the standpipe could be either upwards or downwards. What then becomes important is a relative velocity, the velocity vector difference between solids and gas velocities with reference to the standpipe wall. This observation can then be used to determine the split parameters. Therefore, the determination of the split parameters $b_{l i} \mathrm{~s}$ per second are assumed according to the following relation:

$$
b_{1 i}=1-\frac{1}{\Delta h_{i}}\left(-\frac{U_{s}}{\left(1-\varepsilon_{a v}\right)}+\frac{U_{A(i)}}{\varepsilon_{a v}}\right), i=1,2, \ldots, 4
$$

Eq. (5.33) can also be rewritten as

$$
b_{1 i}=1-\frac{1}{\Delta h_{i}}\left(-\frac{U_{s}}{\left(1-\varepsilon_{a v}\right)}-\left(-\frac{U_{A(i)}}{\varepsilon_{a v}}\right)\right)
$$

The interpretation of $b_{l i}$ s might be made as follows: Choosing the upward direction as positive and the downward direction as negative, the term $\left(-\frac{U_{s}}{\left(1-\varepsilon_{a v}\right)}-\left(-\frac{U_{A(i)}}{\varepsilon_{a v}}\right)\right)$ says that both the solids and gas are flowing downward with the solids moving faster than gas. Accordingly, $b_{1 i}$ s indicate that the remaining solids and gas are flowing upwards. However, from Eq. (5.34), the values of $b_{1 i} \mathrm{~s}$ are not known whether they fall within the range from -1 to 1 during simulation, the following criteria should be adopted. 
$b_{1 i}= \begin{cases}-1 & \text {, if } b_{1 i}(k)<-1 \\ b_{1 i}(k) & \text {, if }-1 \leq b_{1 i}(k) \leq 1, b_{1 i} \neq 0 \quad \text { as there is a continuous feed of gas } \\ 1 & \text {,if } b_{1 i}(k)>1\end{cases}$

\subsection{Results and Discussions}

The case study comprises of the glass beads bed material with a particle density of $2426 \mathrm{Kg} / \mathrm{m}^{3}$, the surface volume diameter of $61 \mu \mathrm{m}$ and the particle sphericity of 0.89 . L-valve and riser aerations are kept fixed at $0.029 \mathrm{~m} / \mathrm{s}$ and $3.66 \mathrm{~m} / \mathrm{s}$, respectively. The amplitude of a sine function of move air's flow rate is $0.055 \mathrm{~m} / \mathrm{s}$ and the period is maintained at 90 seconds. For this experiment, the cold flow circulating fluidized bed riser was operated near the fast fluidized regime, Shadle et. al. [23]. The dynamics of the cold model was extremely rich while operating under this regime with the given bed material, hence, it is quite natural to validate the technique derived in this work under such an operating condition.

\section{6.a Split Estimation}

From Eq. (5.20), it is clear that we are estimating the parameter $\hat{b}$ rather than $\hat{b}_{\text {split }}^{1}$. To determine the split of a move air flow between the two components of a CFCFB, the split parameter $\hat{b}_{\text {split }}^{1}$ should be kept between 0 and 1 . The value 0 indicates that all the aeration at the bottom of the standpipe flows to the L-valve and 1 shows that whole amount of move air flows to the standpipe. During the simulation, the split parameter is maintained within the $0.5-1$ range. This indicates that either $50 \%$ or $100 \%$ move air flows to the standpipe. Hence, finding $\hat{b}_{1}$ from Eq. (5.11) and the above proposition for $\hat{b}_{\text {split }}^{1}$ to estimate $\hat{b}$ are required to successfully estimate the split parameter $\hat{b}_{\text {split }}^{l}$.

For a recursive algorithm (5.20), the initial values of $\hat{\theta}(0), P(0)$ and $\varphi(0)$ are required. The choice of these initial values is well discussed in Ljung et. al. [50]. In this work, they are determined from the rpem routine present in the System Identification Toolbox under the MATLAB environment. In the rpem routine, the default values of initial $\hat{\theta}, P$ and $\varphi$ are all zeros, $10^{4}$ times the unit matrix and $\left\lfloor\Delta P_{\text {bottom }, S P}(1) \quad U_{\text {MoveAir }}(1)\right\rfloor$, respectively. The forgetting factor $\lambda$ is chosen as 0.99 . After several simulations, the discretization factor $\Delta k$ is chosen to be 0.015 seconds to ensure the model fit ( $88 \%$ ) between the estimated and measured pressure drops be maximized, Rupen et. al. [28].

Keeping the split parameter $\hat{b}_{\text {split }}^{1}$ within the 50\%-100\% range gives the split of a move air flow for the glass beads bed material as shown in Fig. (5.2). The estimated pressure drop compared with the measured pressure drop across the bottom of the standpipe is shown in Fig. (5.3). The estimated pressure drop based on move air flow between $50 \%$ and $100 \%$ range thus nicely matches the measured values. Some mismatch might be due to the assumption made for $b_{l}(t)$ in Eq. (5.11), being the average mass flux at the bottom of the standpipe. 


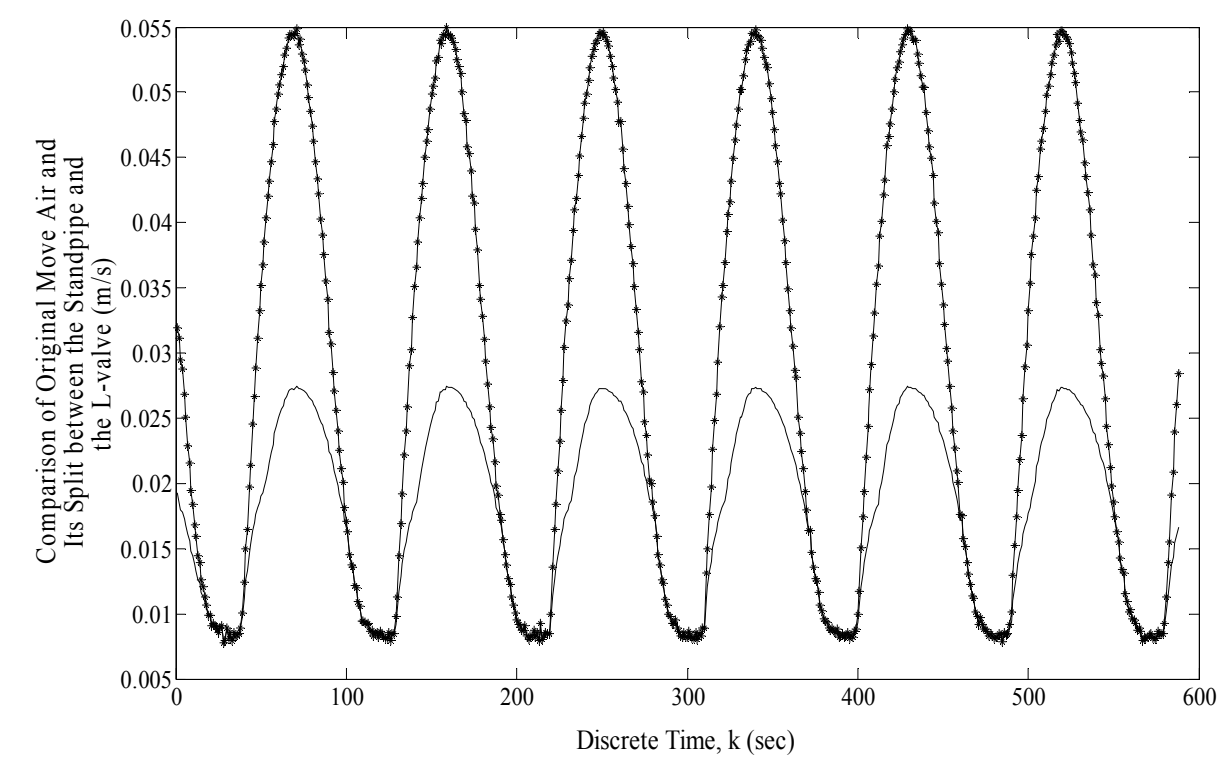

Figure (5.2): A comparison plot of original move air and its split between the standpipe and the Lvalve. Original move air flow fed at the bottom of the standpipe just above the non-mechanical valve is indicated by a black solid line with stars and the amount of move air that flows up the standpipe is represented by a black solid line.

To verify if this split is indeed correct, the L-valve pressure drop is estimated with remaining move air flow using the RPEM (20) on model (5.28). The comparison with the measured values is depicted in Fig. (5.4) and the estimation is perfect (model fit 99\%) in this case as illustrated.

The measured values are represented by a black solid line with stars and the estimated values are indicated by a black solid line. Both the values are well matched as shown in these figures and thus, considering the estimate of both the pressure drops based on a move air flow split, that agree nicely with the measured values, justifies that the split is correct when the CFCFB operates near the fast fluidized regime. 


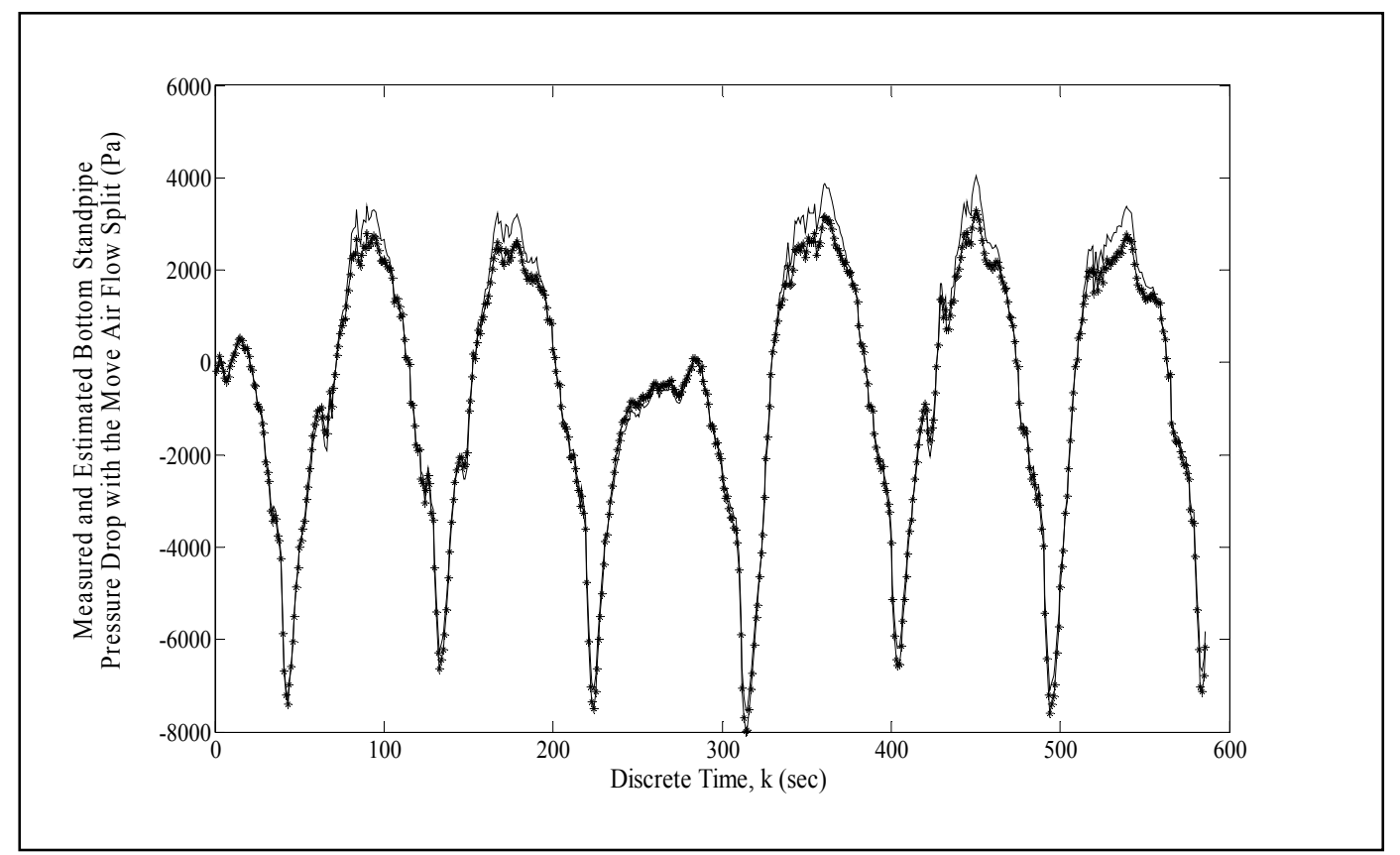

Figure (5.3): A comparison plot of measured and estimated bottom standpipe pressure drop based on more than $50 \%$ move air flow.

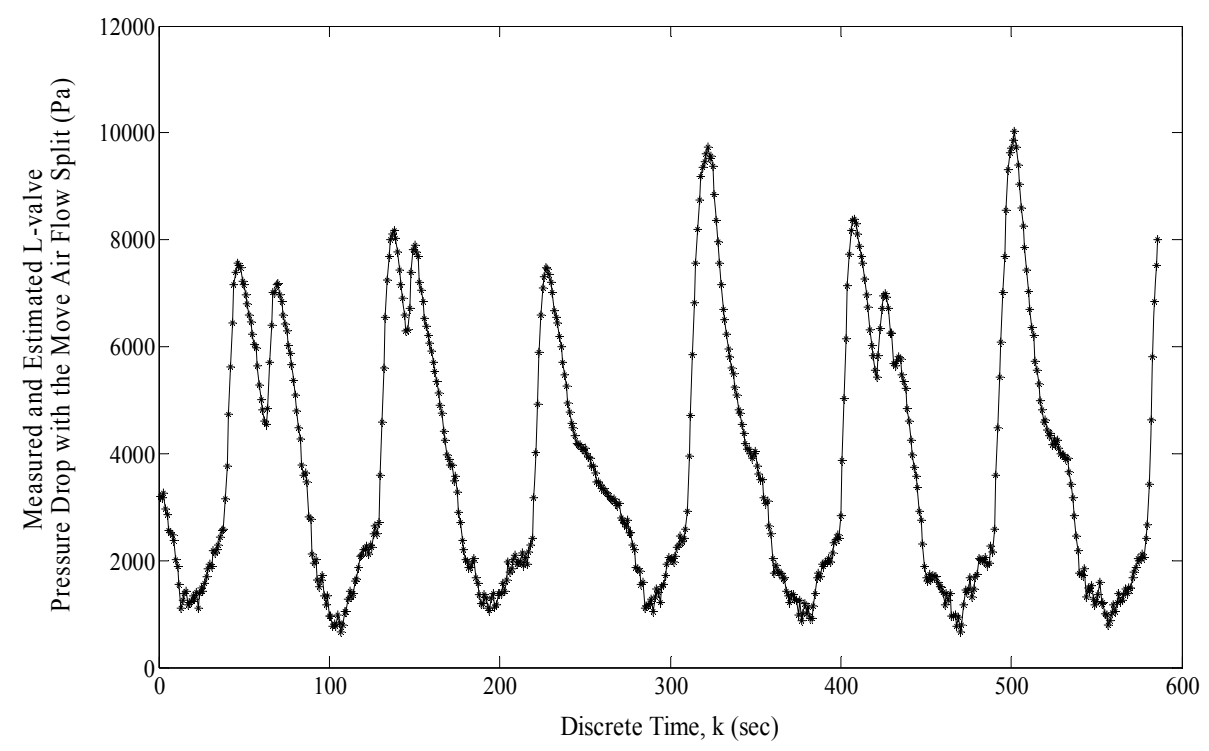

Figure (5.4): A comparison plot of measured and estimated L-valve pressure drop based on less than $50 \%$ move air flow. 


\section{6.b Route Estimation}

The above simulation results justify the assumption made in the beginning for a move air flow being flowing upwards with respect to the downward flowing solids. To further support this deduction, we can analyze a plot of move air split parameter at the location where the spiral vane is placed as shown in Fig. (5.5). It is clear that only $0.022 \%$ (maximum) move air after splitting between the standpipe and the Lvalve flows in the downward direction when we consider the split parameter at that location. However, it is not always the case as illustrated that the move air flows only in the upward direction during the entire operation period but it also flows in the downward direction at intervals even though its magnitude is small.

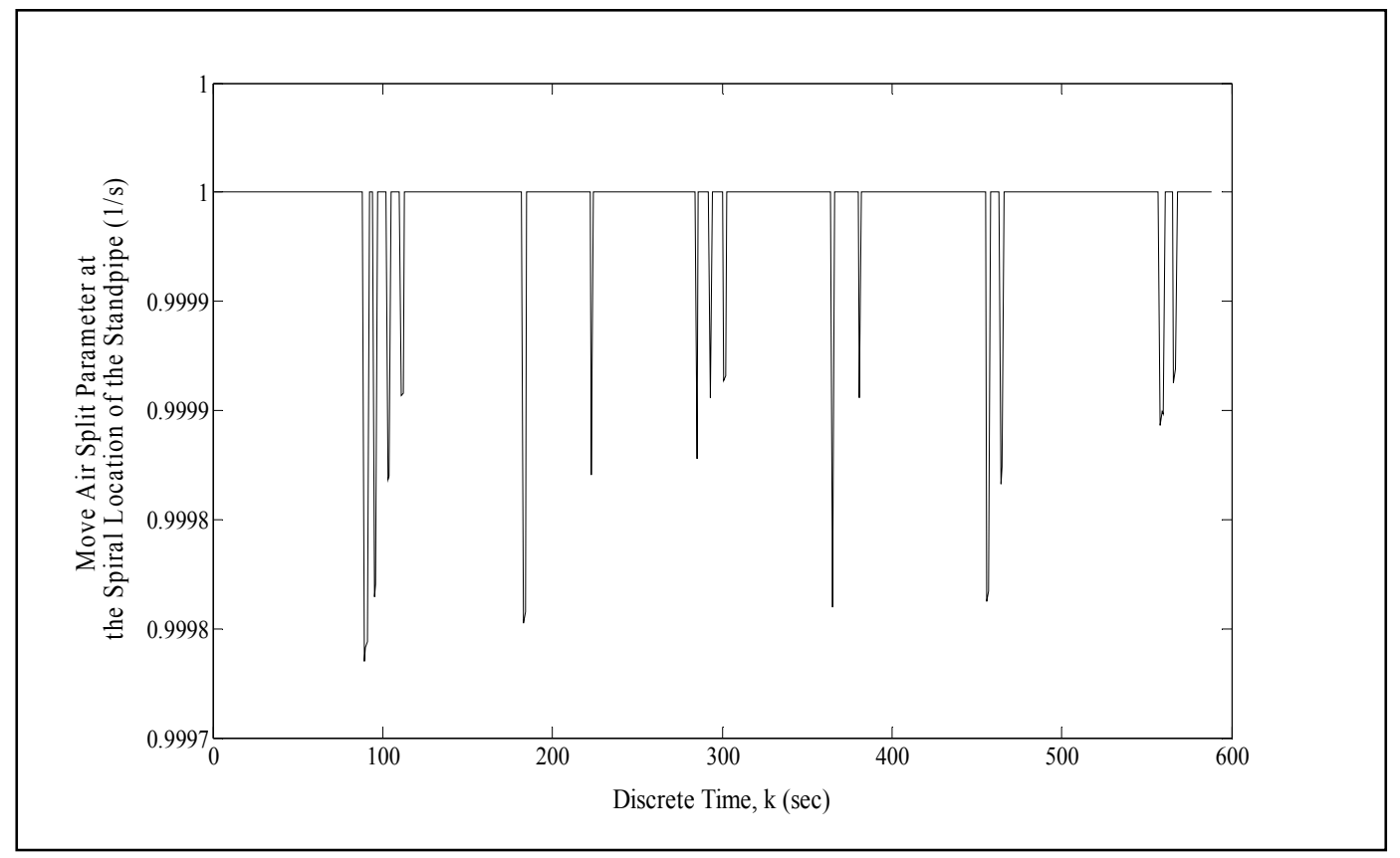

Figure (5.5): A plot of move air split parameter per second at the location where the spiral vane is placed within the standpipe.

Similar inference can be arrived at with other aeration flows within the standpipe. A plot of additional aerations fed across the standpipe and their split are shown in Fig. (5.6). These aerations $U_{A 1}, U_{A 2}, U_{A 3}$ and $U_{A 4}$ were maintained at the mean level of $0.0077 \mathrm{~m} / \mathrm{s}, 0.0067 \mathrm{~m} / \mathrm{s}, 0.01 \mathrm{~m} / \mathrm{s}$ and $0.0065 \mathrm{~m} / \mathrm{s}$ respectively, during operation. Measured aerations are represented by a solid purple line with stars and the splits are indicated by solid black lines. Only that time interval is shown where the flows took place in the downward direction with each aeration. Comparing to move air flow, higher amounts of the additional aerations travel down the standpipe during operation. Maximum quantities are respectively $1.35 \%, 1.1 \%$, $1.1 \%$ and $0.38 \%$ for the aerations progressively at positions above the move air location. Just like move air flow, these aerations are mostly moving in the upward direction and moving downward at some time intervals.

Finally, the pressure drop estimate using the RPEM on the model (5.31) is compared with the measured pressure drop as presented in Fig. (5.7). As with the bottom standpipe pressure drop estimate and that of the L-valve pressure drop, the estimated pressure drop at the spiral location based on the splits of the aerations including move air flow fits adequately with the measured values. The measured values are 
depicted by a solid black line with stars and the estimates are indicated by a black solid line. The model fit defined in [28] for the present case is $94 \%$.

Thus, the proposed model of the pressure drops at the base of the standpipe and across the L-valve makes it possible to determine an actual split of the move air flow between the two components of a CFCFB at a given moment of time, with the aid of recursive prediction error method. Compared to the measured pressure drops, this instrumental technique provides the estimates as a function of a split of move air flow, that are nicely matched, and therefore verifies the split is correctly predicted. Based on the case study, it can be concluded that more than $50 \%$ of a move air fed at the bottom of the standpipe flows to the standpipe and the remaining quantity passes to the L-valve.

Similarly, the pressure drop at the location where the spiral vane is placed is estimated using splits of aerations fed across the standpipe including move air flow to justify if their routes within the standpipe at that particular location are acceptable by comparing it with the measured pressure drop. It was shown that all these aerations flow in higher amounts towards the upper section of the standpipe by choosing the split parameters for each aeration as a slip velocity divided by the height of the respective locations and forcing them to lie within -1 and 1 range. If the path of these aerations are required at different locations other than the considered one, similar technique could be utilized and justified by estimating and comparing the pressure drop obtained from these splits at that location with the measured pressure drop.

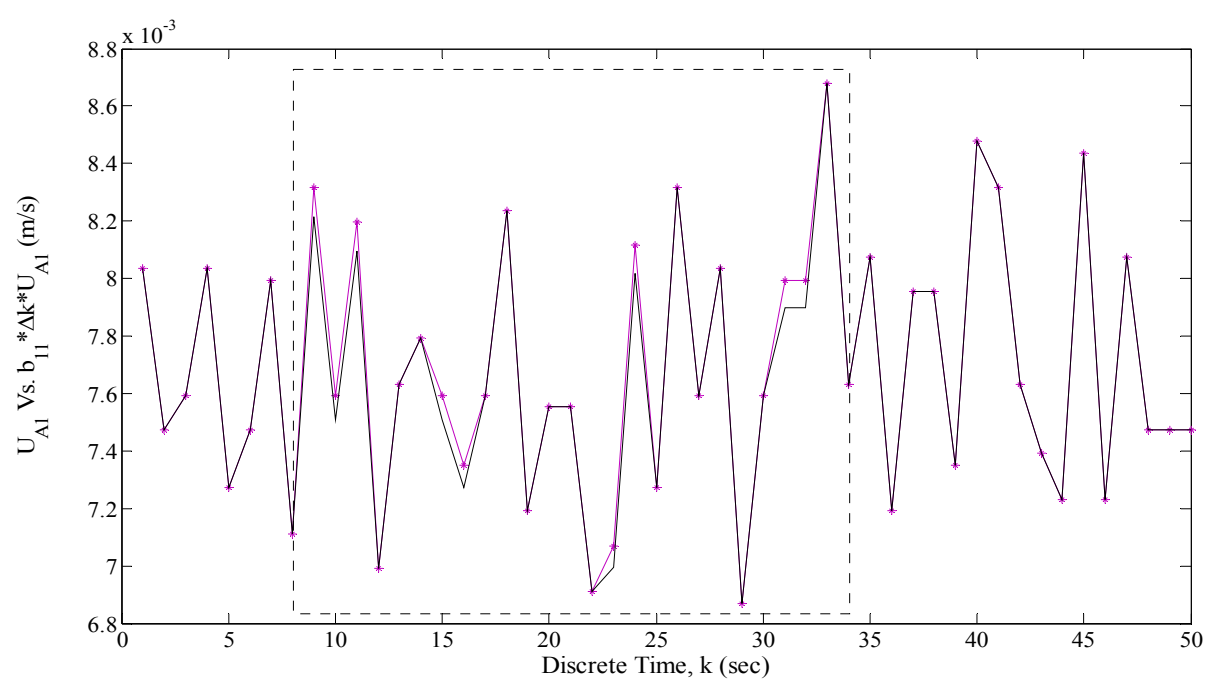



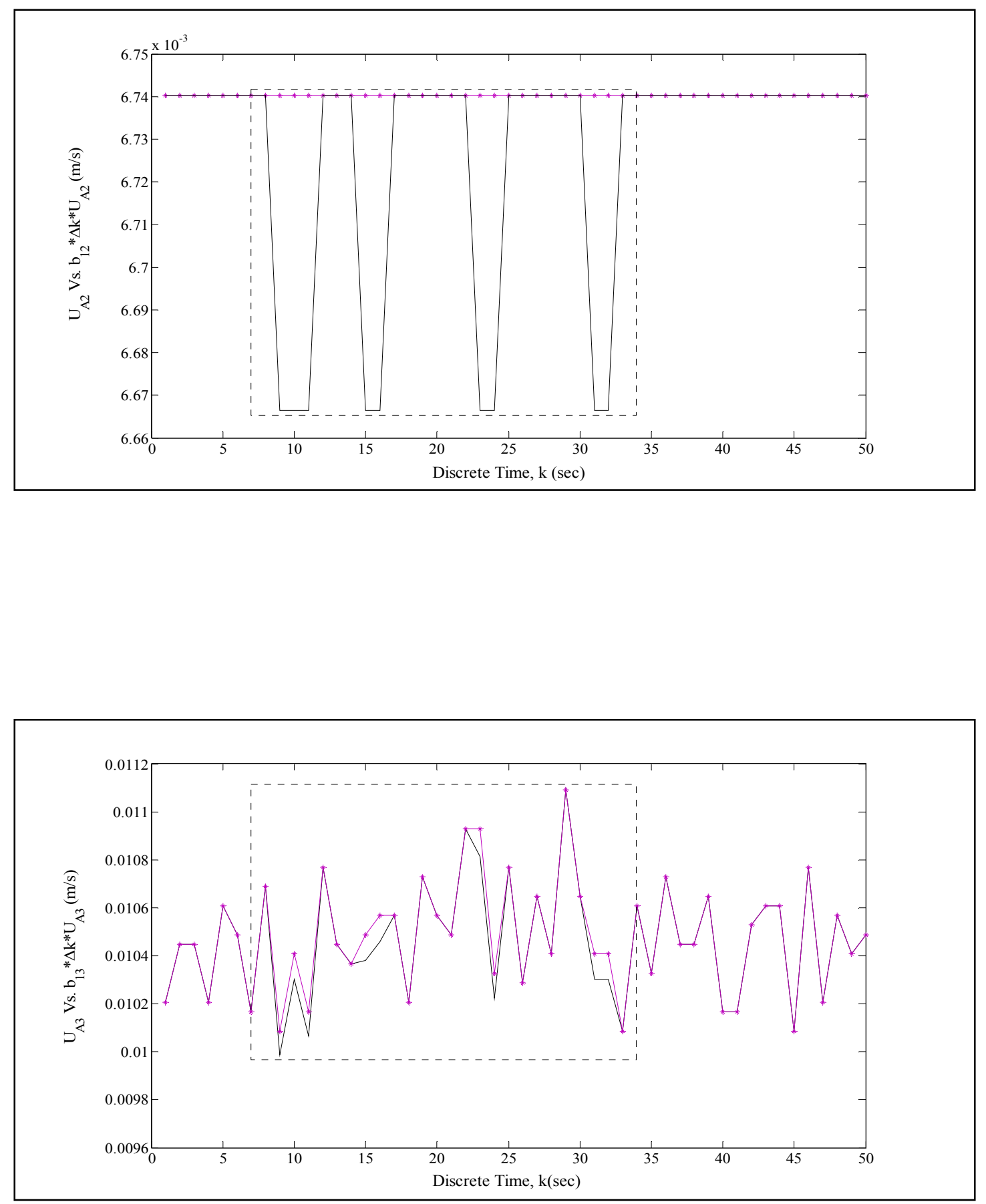


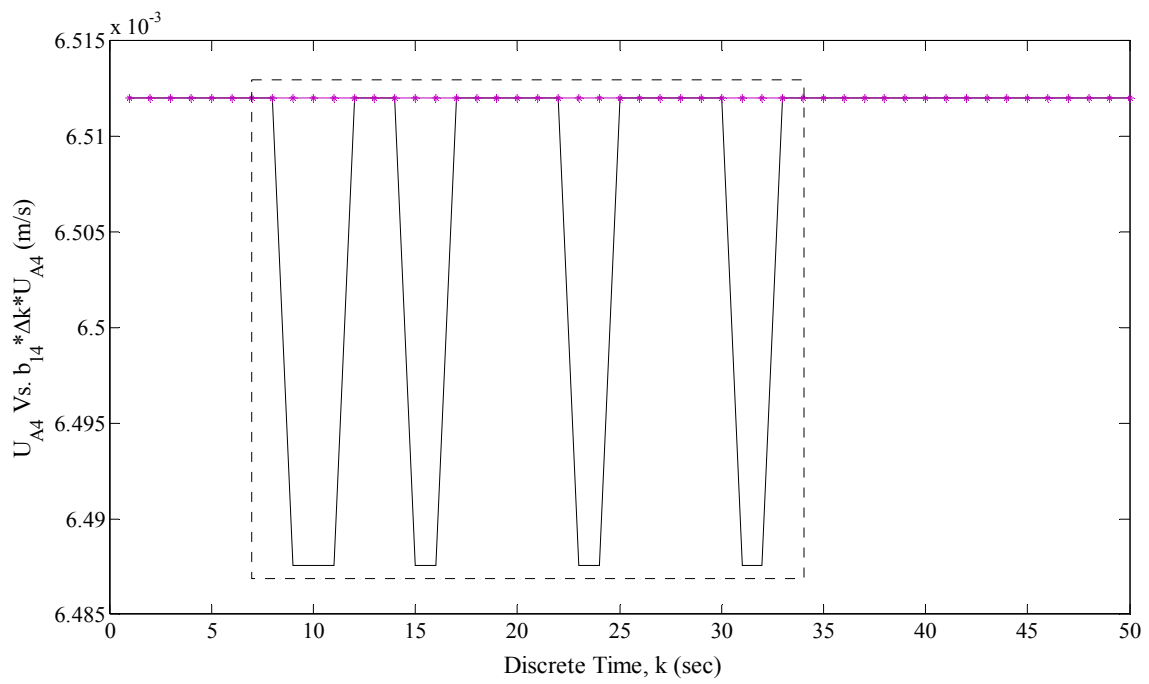

Figure (5.6): A comparison plot of additional aeration flows and their routes within the standpipe.

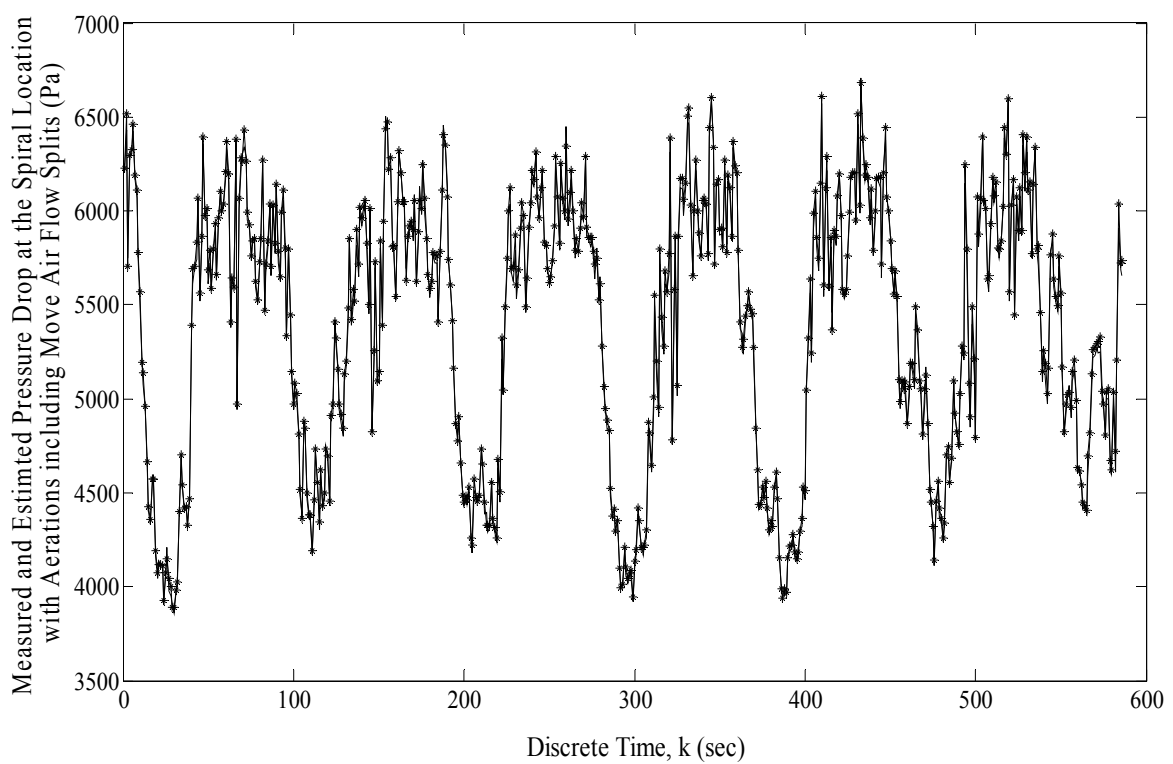

Figure (5.7): A comparison plot of measured and estimated pressure drop at the spiral location based on aerations including move air flow splits. 


\section{Chapter 6}

\section{Adaptive PID Control of a Cold Flow Circulating Fluidized Bed}

\subsection{Motivation}

It is interesting to note that more than half of the industrial controllers in use today utilize PID control schemes. Many different types of tuning rules for controller parameters have been proposed in the literature, Ziegler et. al. [51], Astrom et. al. [52]. This has moved from ad hoc tuning methods such as Ziegler and Nichols tuning tables to pole placement, frequency response. Also, automatic tuning methods have been developed and some of the PID controllers may possess online automatic tuning capabilities, Astrom et. al. [53].

In Gao [29], the tuning of a PID controller is reduced to adjusting a single parameter known as the bandwidth optimization parameter, instead of three. Practical optimization is incorporated into the oneparameter tuning derived from a controller performance. This is subjected to designing PID control system based on a good understanding of the plant under study and its environment.

However, in number of instances, the plant to be controlled such as the cold flow circulation fluidized bed is too complex and the basic physical processes in it are not fully understood. Control design techniques then need to be augmented with an identification technique aimed at obtaining a progressively better understanding of the plant to be controlled. It is thus intuitive to aggregate system identification and control. If the system identification is recursive - that is the plant model is periodically updated on the basis of previous estimates and new data - identification and control may be performed concurrently.

In this chapter, a technique of Recursive Least Squares Estimation (RLS) is applied to obtain a model of the process and its environment from input-output experiments and this model is used to design a PID controller with regards to a certainty equivalence principle. The bandwidth parameter of the controller is automatically adjusted during the operation of the plant as the amount of data available for plant identification increases. The technique is illustrated by controlling the solids flow rate of the cold flow circulating fluidized bed present at NETL.

\subsection{An Adaptive PID Control Algorithm}

Consider a common controller design scenario in Fig. (6.1). This design method is summarized by a controller structure, and a relationship between plant parameters and controller parameter, that is bandwidth optimization parameter, $\omega_{c}$. Since the plant parameters are in fact unknown, they are obtained using a RLS algorithm. The controller parameter $\omega_{c}$ is then obtained from the estimates of the plant parameters, in the same way as if these were the true parameters. 


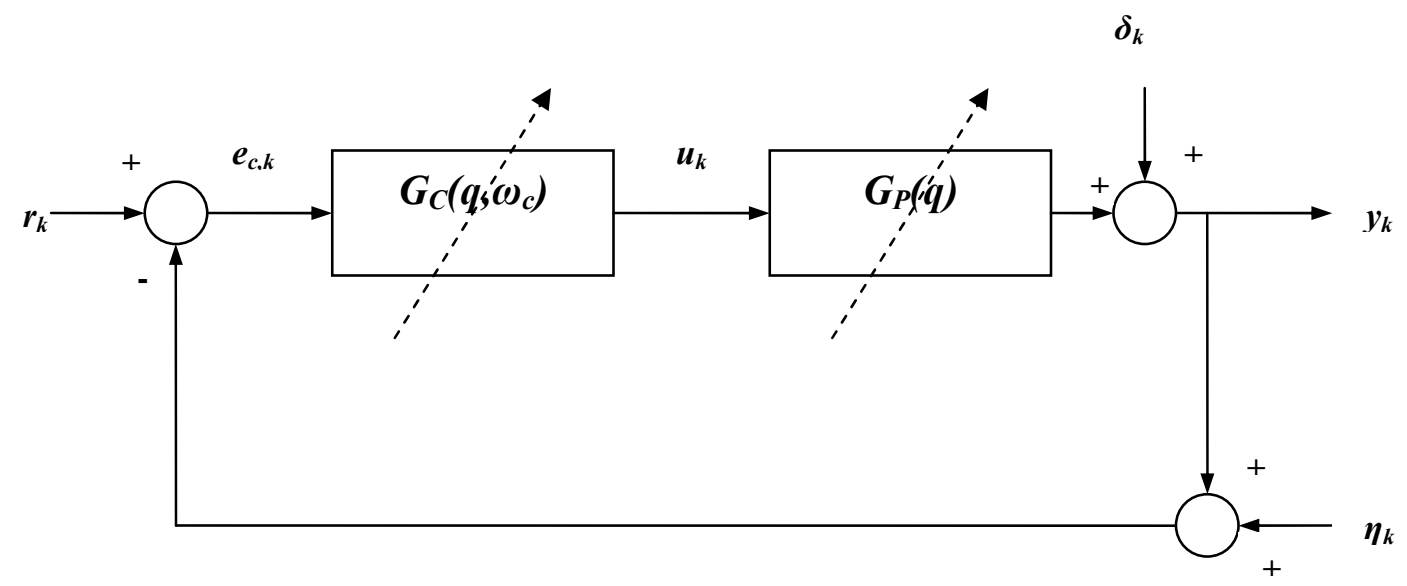

Figure (6.1): Common controller design scenario.

Let the plant be specified as

$A(q) y(k)=B(q) u(k)+C(q) v(k)$

where

$v(k)=\delta(k)+\eta(k)$

$A(q)=q+a(k)$

$B(q)=b(k)$

$C(q)=1$

Only first order single input single output system is considered. Similar solutions for higher order multiinput single-output plants can be easily obtained. Here, $q$ is the forward time shift operator and $k$ is the discrete time index.

The model of the plant can be written as

$\hat{A}(q) \hat{y}(k)=\hat{B}(q) u(k)$

This gives

$\hat{y}(k)=\hat{G}_{P}(q) u(k)$

where $\hat{G}_{P}(q)$ is the estimated plant transfer function given as 
$\hat{G}_{P}(q)=\frac{\hat{b}(k)}{q+\hat{a}(k)}$

In the controller design, $\omega_{c}$ is selected as the measure of performance of the controller, [29]. $\omega_{c}$ is denoted as the bandwidth of the feedback controller and $\hat{G}_{C}\left(q, w_{c}\right)$ is the controller. Bandwidth parameterization also known as $\omega_{c}$ - parameterization refers to assigning all closed-loop poles at $-\omega_{c}$ and making all parameters of the controller a function of $\omega_{c}$. Applying the simple pole-placement design to the first order plant (6.4), a $\omega_{c}$ - parameterized controller is obtained as

$G_{C}\left(q, \omega_{c}\right)=\frac{\omega_{c}(q+\hat{a}(k))}{\hat{b}(k) q}$

making the closed loop transfer function $\omega_{c} / q+\omega_{c}$.

From the figure, it is clear that

$u(k)=G_{c}\left(q, \omega_{c}\right) e_{c}(k)$

Substituting the controller transfer function from Eq. (6.5) to Eq. (6.6) and manipulating, we obtain

$u(k)=\frac{\omega_{c}\left(e_{c, k}+\hat{a}_{k-1} e_{c, k-1}\right)}{\hat{b}_{k-1}}$

and

$u(k-1)=\frac{\omega_{c}\left(e_{c, k-1}+\hat{a}_{k-2} e_{c, k-2}\right)}{\hat{b}_{k-2}}$

Substituting the value of $u(k-1)$ in Eq. (6.2) gives

$\hat{y}_{k}=-\hat{a}_{k-1} \hat{y}_{k-1}+\hat{b}_{k-1} u(k-1)=-\hat{a}_{k-1} \hat{y}_{k-1}+\frac{\hat{b}_{k-1} \omega_{c}\left(e_{c, k-1}+\hat{a}_{k-2} e_{c, k-2}\right)}{\hat{b}_{k-2}}$

\section{3 $\omega_{c}$ Estimation}

The following cost function is minimized with respect to $\omega_{c}$ at $\omega_{c}=\hat{\omega}_{c}$.

$J_{C}=(r(k)-\hat{y}(k))^{2}$

This gives 


$$
\hat{\omega}_{c}=\left(\bar{b}^{T} \bar{b}\right)^{-1} \bar{b}^{T} \ell_{k}
$$

where

$$
\bar{b}=\frac{\hat{b}_{k-1}}{\hat{b}_{k-2}}\left(e_{c, k-1}+\hat{a}_{k-2} e_{c, k-2}\right)
$$

and

$$
\ell_{k}=r(k)+\hat{a}_{k-1} \hat{y}_{k-1}
$$

\subsection{Summary of Adaptive PID Control Algorithm}

a) At $k=0$, initialize $\hat{a}_{0}, \hat{a}_{-1}, \hat{b}_{0}, \hat{b}_{-1}, \hat{e}_{c, 0}, \hat{e}_{c,-1}, \hat{y}_{0}$ and $P_{0}$. Assume $\lambda$.

b) At $k=1,2, \ldots, N-1$,

i. $\quad \ell_{k}=r(k)+\hat{a}_{k-1} \hat{y}_{k-1}$

ii. $\quad \bar{b}=\frac{\hat{b}_{k-1}}{\hat{b}_{k-2}}\left(e_{c, k-1}+\hat{a}_{k-2} e_{c, k-2}\right)$

iii. $\quad \hat{\omega}_{c}=\left(\bar{b}^{T} \bar{b}\right)^{-1} \bar{b}^{T} \ell_{k}$

iv. $\quad \hat{\theta}_{k-1}=\left[\begin{array}{ll}-\hat{a}_{k-1} & \hat{b}_{k-1}\end{array}\right]^{T}$

v. $\quad u(k-1)=\frac{\hat{\omega}_{c}\left(\hat{e}_{c, k-1}+\hat{a}_{k-2} \hat{e}_{c, k-2}\right)}{\hat{b}_{k-2}}, \quad \varphi_{k}^{T}=\left[\begin{array}{ll}\hat{y}_{k-1} & u(k-1)\end{array}\right]$

vi. $\quad Q_{k}=\frac{P_{k-1}}{\lambda+\varphi_{k}^{T} P_{k-1} \varphi_{k}}$

vii. $\quad K_{k}=Q_{k} \varphi_{k}$

viii. $\quad \hat{y}_{k}=\varphi_{k}^{T} \hat{\theta}_{k-1}$

ix. $\quad \hat{\theta}_{k}=\hat{\theta}_{k-1}+K_{k}\left(r_{k}-\hat{y}_{k}\right)($ a $d x l$ vector $)$

x. $\quad P_{k}=\frac{1}{\lambda}\left(P_{k-1}-\frac{P_{k-1} \varphi_{k} \varphi_{k}^{T} P_{k-1}}{\lambda+\varphi_{k}^{T} P_{k-1} \varphi_{k}}\right)$ 
c) $\hat{a}_{k}=\hat{\theta}_{k}(d-1), \hat{b}_{k}=\hat{\theta}_{k}(d), \hat{a}_{k-2}=\hat{a}_{k-1}, \hat{a}_{k-1}=\hat{a}_{k}, \hat{b}_{k-2}=\hat{b}_{k-1}, \hat{b}_{k-1}=\hat{b}_{k}$

$$
\hat{e}_{c, k-2}=\hat{e}_{c, k-1}, \hat{e}_{c, k-1}=\hat{e}_{c, k}=\left(r_{k}-\hat{y}_{k}\right), \hat{y}_{k-1}=\hat{y}_{k}, \hat{\theta}_{k-1}=\hat{\theta}_{k}, P_{k-1}=P_{k}
$$

d) Repeat steps (b) and (c) until $k=N$.

\subsection{Adaptive PID Control of a CFCFB}

The algorithm proposed in this chapter was used for control of a cold flow circulating fluidized bed (CFCFB) present at NETL.
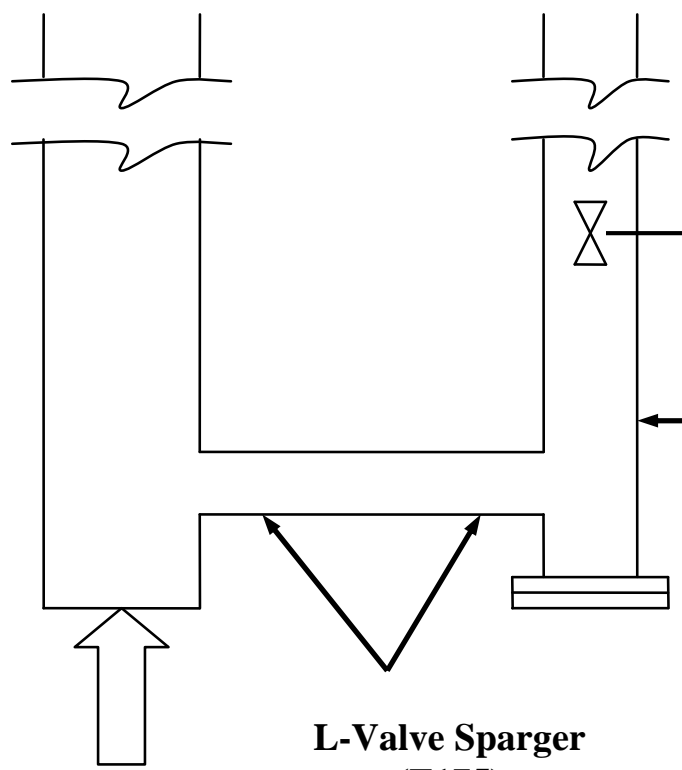

\section{Riser Aeration}

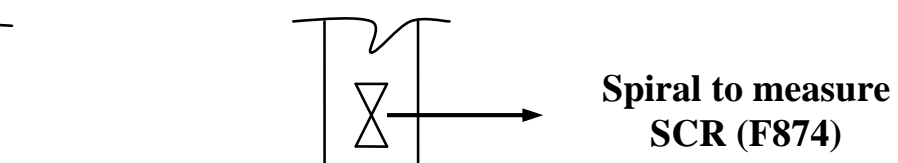

(F431)

Figure (6.2): The cold flow circulating fluidized bed test plant. Only part of it required for control is shown

The bottom portion of a given set up for control purpose is illustrated in Fig. (6.2). The mathematical model of the fluidized system based on a structural identification (the primary step of system identification) was derived as

$$
\hat{y}_{k}=-\hat{a}_{k-1} \hat{y}_{k-1}+\left[\begin{array}{lll}
\hat{b}_{1}(k-1) & \hat{b}_{2}(k-2) & \hat{b}_{3}(k-1)
\end{array}\left[\begin{array}{l}
u_{1}(k-1) \\
u_{2}(k-1) \\
u_{3}(k-1)
\end{array}\right]\right.
$$

where $\hat{y}$ is the output solids circulation rate (SCR) in $\mathrm{Kg} / \mathrm{s}, u$ is the input vector consisting of a move air flow injected into the stand pipe just above the entrance of an L-valve, the L-valve aeration and the high aeration at the base of the riser that causes solids to flow up the riser column. All the inputs are measured in $\mathrm{m} / \mathrm{s}$. 
The experimental condition is same as considered in Chapter 5. However, additional aerations fed across the standpipe are not considered for the control purpose.

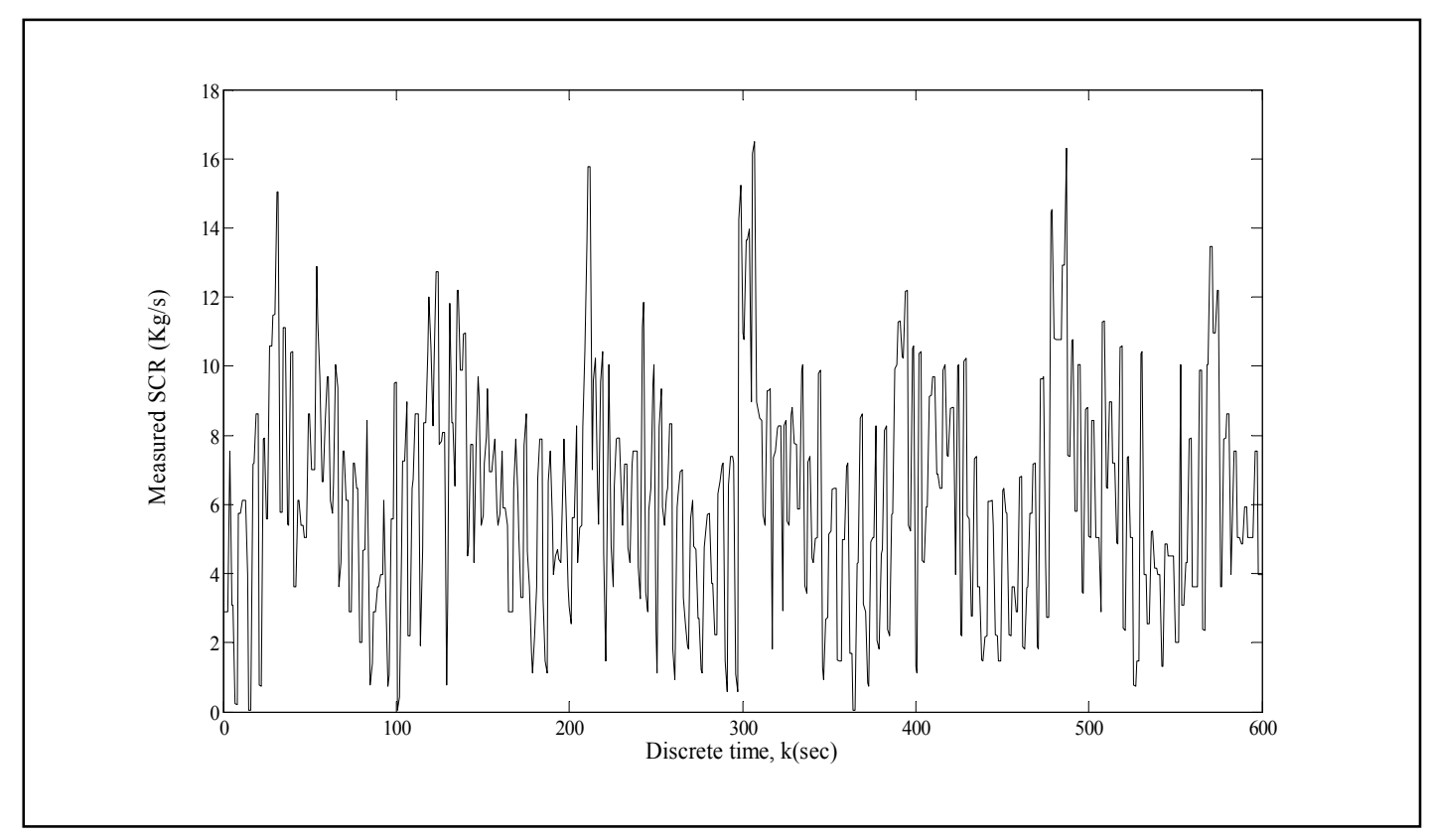

Figure (6.3): Measured solids circulation rate (SCR) (black solid line).

Under the operating regime mentioned, the CFCFB behaved like a nonlinear chaotic system. This can be rationalized by analyzing pressure fluctuation time series measured across various sections of the device. The solids circulation rate measured by a spiral vane placed in a packed bed portion of the standpipe Ludlow et. al. [20], seems to be contaminated by noise as depicted in Fig. (6.3). The design objective is to control solids circulation rate subjected to a smooth sinusoidal reference signal of magnitude $6.17 \mathrm{Kg} / \mathrm{s}$.

The reference signal was generated from a solids superficial velocity, $U_{s}$ that is obtained from an Knowlton's correlation Knowlton et. al. [48], between the packed bed and fluidization condition, for a slip velocity between gas and solids and assuming solids travel faster than gas in the downward direction as follows:

$$
\begin{aligned}
& U_{s}=\frac{U_{g}}{\varepsilon_{\text {ave }}}\left(1-\varepsilon_{\text {ave }}\right)+\frac{U_{m f}}{\varepsilon_{m f}}\left(1-\varepsilon_{\text {ave }}\right)\left(\varepsilon_{\text {ave }}-\varepsilon_{p b}\right), \\
& r(k)=\rho_{p} A_{S P}\left(1-\varepsilon_{\text {ave }}\right) U_{s}, \\
& \varepsilon_{\text {ave }}=\frac{\varepsilon_{m f}+\varepsilon_{p b}}{2} \\
& U_{g}=u_{l}(\text { MoveAirFlow })
\end{aligned}
$$


The initial values of model parameters and the error values between measured and estimated output, are obtained from the input-output data available by running the plant for 9 seconds using RLS algorithm on the multi-input single-output system (6.14) with a forgetting factor $\lambda=0.99$. Simulated result is illustrated in Fig. (6.4) and the error between the desired signal and the controlled output is shown in Fig. (6.5). As can be seen from the Fig. (6.4), the two are almost indistinguishable. We conclude that for the given example high performance is achieved through the use of an adaptive PID controller.

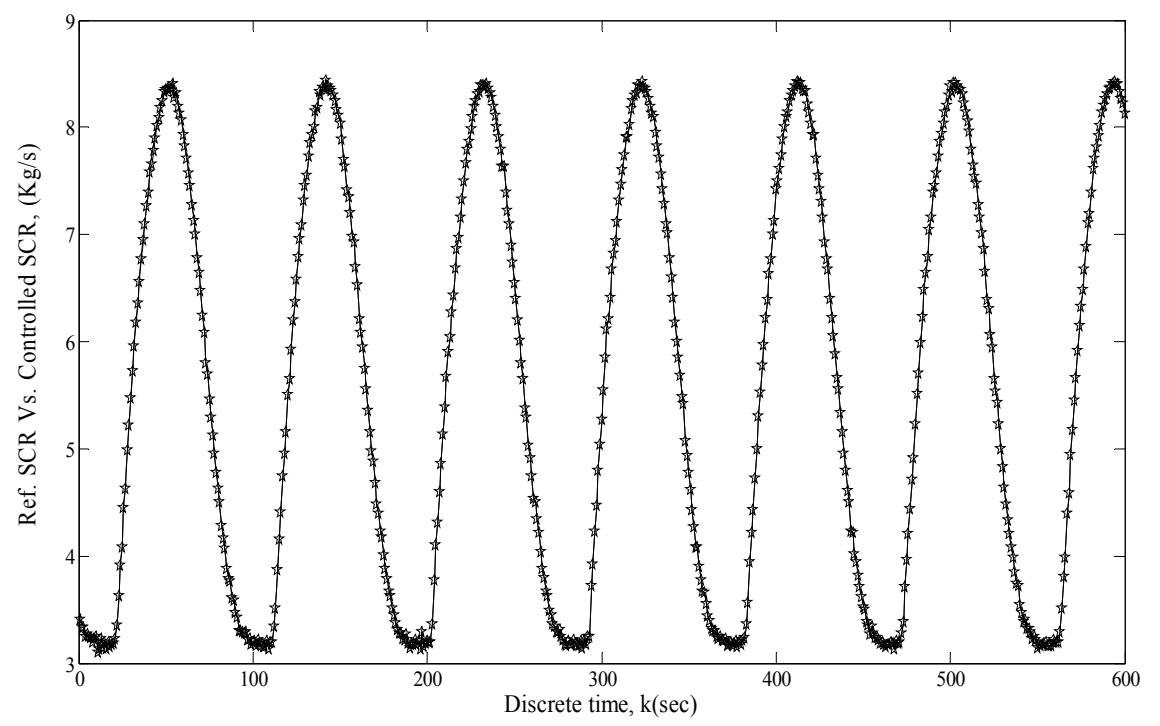

Figure (6.4): Reference SCR (black solid line) and controlled SCR (black dotted line).

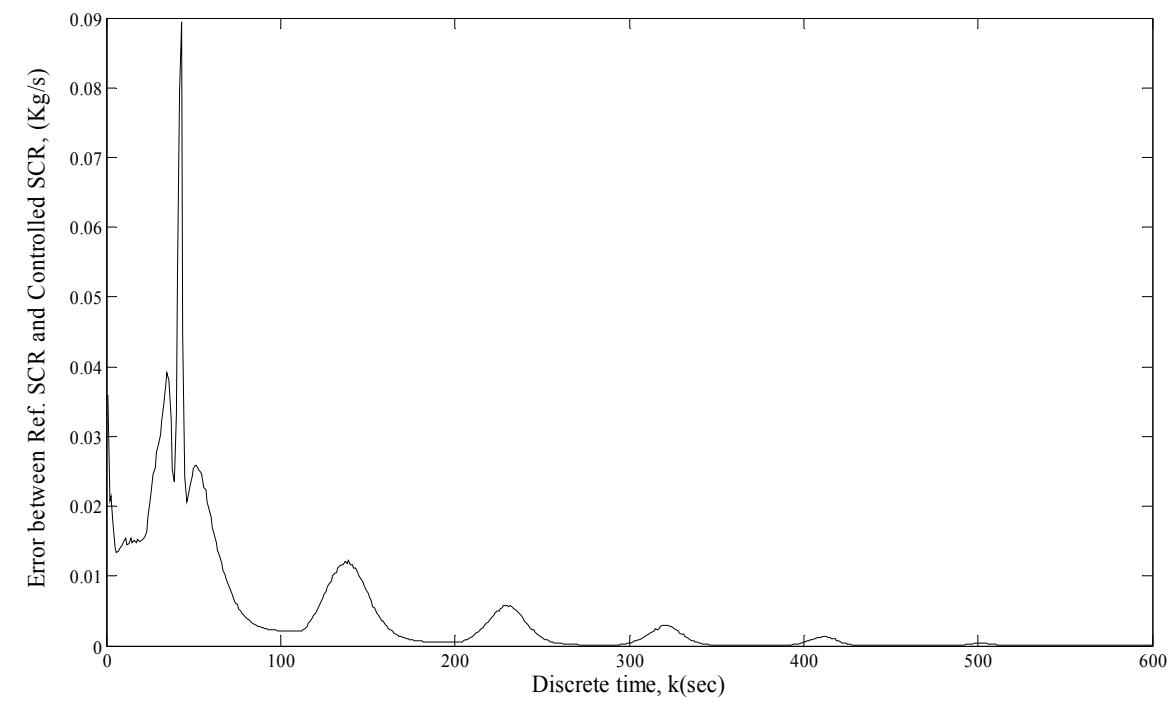

Figure (6.5): Error (black solid line) between reference SCR and controlled SCR. 
Summarizing, we have proposed a new technique for the PID control of plants whose mathematical models are not available. The new method is based on the theory of Recursive Least Squares Estimation and allows determining the bandwidth optimization parameter that has reduced the tuning of a PID controller to adjusting a single parameter instead of three as well as the closed loop model parameters online. The method was tested on an illustrative example to illustrate its performance and has incorporated real world scenarios such as sensor noise, disturbance and the lack of mathematical model. 


\section{Chapter 7}

\section{Conclusions and Recommendations}

Usually, a complex process like the cold flow circulating fluidized system consists of interconnected sub models. Based on multiple model identification technique, modeling of each sub model from the inputs chosen as move air flow, riser aeration and total riser pressure drop and the solids circulation rate as the output gives a global description of the CFCFB's input-output behavior.

Normalized radial basis functions were used as weighting functions that combine the local linear statespace models to form a global model for the CFCFB. A regularized nonlinear optimization-based method was used to identify multiple models scheduled by the inputs, and the centers and widths of the radial basis functions. Measurement noise and a certain kind of process noise were taken into consideration. The nonuniqueness of the fully parameterized state-space representation was handled by calculating at every iteration the direction in which a change of parameters does not influence the input-output behavior of the model, and projecting these directions out of the parameter update. To initialize the nonlinear predictor during estimation, a global linear model was estimated from N4SID and used to initialize all multiple models; in addition the weighting functions' centers were initially estimated using fuzzy C-means clustering algorithm and the widths were uniformly distributed over the operating range of the scheduling vector.

This work has been carried out to determine the models for future control purpose of the CFCFB using a model predictive control technique, Townsend et. al. [54], that is widely used in industry. In addition, the approach based on dividing the whole operating range of the nonlinear system into different operating regimes and defining a local mechanistic model in each operating regime of the CFCFB can be used to obtain the global phenomenological model. This may, in addition, require the use of different tree algorithms like CART and/or MARS for dividing the data sets into different operating regimes. Besides, an algorithm developed by Aguirre et. al.[55] can be used to estimate fixed points of a nonlinear dynamic system like the CFCFB from time series at each operating regime.

The present work is obtained for the offline case and the iterative optimization algorithm converges at around 15 iterations. The time required for these iterations on a $3.0 \mathrm{GHz}$ Intel-Windows PC is 25 minutes. It is believed that this can be improved if it is possible to choose a linear parameterization of a described predictor in the optimization problems such that high accuracy is obtained with a low computational complexity and a tradeoff can be made between accuracy and complexity. The optimization problem that is nonlinear, nonconvex in multiple model identification then becomes the convex problem with linear parameterization where the model can be parameterized with system-based basis functions, and hence, minimizing the prediction error becomes a least squares problem that can be solved analytically. Such identification provides the possibility to improve the estimated model using iteration. This iteration can be implemented efficiently and can therefore serve as a possible alternative to nonlinear optimization in identification problems for large scale industrial processes like the CFCFB, van Donkelaar et. al. [56].

When a cold flow circulating fluidized bed is believed to operate in a linear fashion as with a present cork bed material, it can be described by a linear time invariant model. Starting from a complete black box modeling based on the interrelationship among dependent and independent parameters using a PEM on a linear predictor and converting it into the form that does not contain any fictitious variable introduced in the initial system identification phase expresses the system under consideration in terms of physical 
variables only. The deterministic as well as stochastic components of a variable of interest can then be analyzed separately. This has been done with the solids circulation rate; the stochastic component was obtained utilizing stochastic components of various pressure drops across the CFCFB and the move air flow, riser aeration and loop seal fluidization air were used to find the deterministic counterpart.

Another parameter that is important for the optimal operation of the CFCFB is the bed height. A new formula for a bed height calculation was derived. A lean phase void fraction is required that was chosen in the range of 0.98 and 1. It can also be estimated from the one-dimensional-dynamic-model of the standpipe developed by Sams [14]. In this model, move air split and other aerations fed across the standpipe is required to estimate the state variable; hence, the split estimation developed in this thesis could be utilized. However, in future, the estimated solids circulation rate should be used rather than measured solids circulation rate considering they are not measurable in a hot unit, to find solids superficial velocity in order to determine split parameters for the aeration flows within the standpipe. The present approach can also be checked when the CFCFB operates with the loop seal in place of the Lvalve.

Once these two important parameters, SCR and bed height, are estimated, a linear model predictive control and PID control techniques could be developed to optimally control the CFCFB. The task of the MPC controller would be to vary the amount of solids circulating in the CFB by varying the move air flow, riser aeration and loop seal (or L-valve) fluidization air as the total amount of solids in the whole system amounts to the mass of solids in the riser, standpipe and loop seal (or L-valve). Similarly, the fluidization within the standpipe is regulated by the aerations fed at four points in the CFCFB. These aerations can be controlled using PID control method by controlling the bed height in the standpipe.

Finally, a novel adaptive PID control algorithm was developed for the system lacking a mathematical model and corrupted by measurement and process noise and was tested on a benchmark CFCFB example. 


\section{Appendix}

\section{A1 Characteristics of Solid Particles Used}

\section{A1.1 General Definitions}

A particle may be defined as a small object having a precise physical boundary in all directions. The particle is characterized by its volume and interfacial surface in contact with the environment.

Solid particles are rigid and have a definite shape. A sphere is a natural choice to define a particle, though most natural particles are not spherical. Hence, natural particles are characterized by their degree of deviation from spherical shape, sphericity, and an equivalent diameter.

Sphericity describes the departure of the particle from a spherical shape. Surface-volume diameter is the diameter of a sphere having the same surface to volume ratio as that of the particle.

In a particulate mass, particles rest on each other due to the force of gravity to form a packed bed. Depending on the shape of particles and packing characteristics, a certain volume of space in between particles remains unoccupied. Such space is called a void volume and is specified as voidage or porosity, defined as

Voidage, $\varepsilon=$ porosity $=$ void volume $/$ volume of (particles and voids)

In such a particulate mass, generally particles are not uniform in size and are characterized by particle size distribution. There are several characteristic properties that define it - Number of particles, Total Surface Area and Total Volume. In fluidization and in most chemical engineering applications, total volume and surface are the two chosen properties to represent the material content and interfacial area across which the transfer processes occur. For a pressure drop through the bed, the surface area is most important. The mean particle size is thus defined in such a way that it equals the average surface area of particles of sizes in the bed.

\section{A1.2 Particle Classification for Cork and Glass Beads}

In the light of fluidization experience, Geldart [57] classified solids broadly under four groups, A, B, C, and $\mathbf{D}$ as shown in Fig. (A1.1). The particle's classification is plotted against the density difference between the solids and the fluidizing gas. This classification is important in understanding the fluidization behavior of solids particles, because under similar operating conditions particles of different groups may behave entirely differently. 


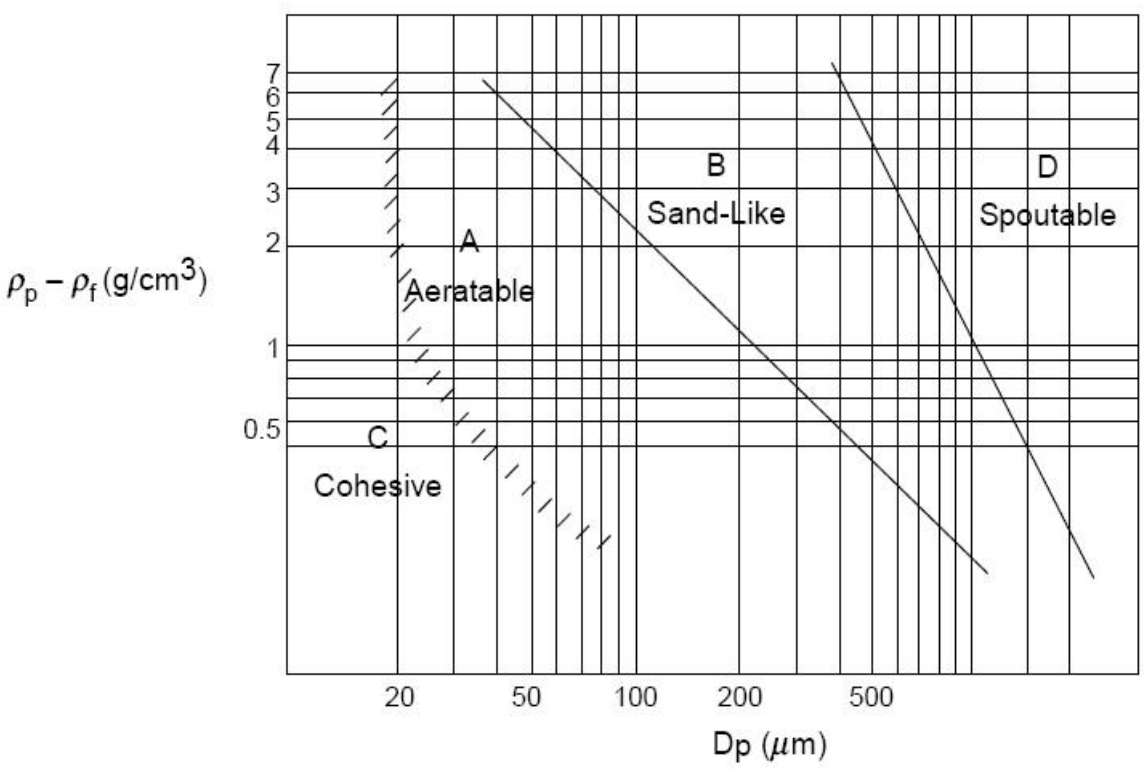

Figure (A1.1): Powder classification developed by Geldart.

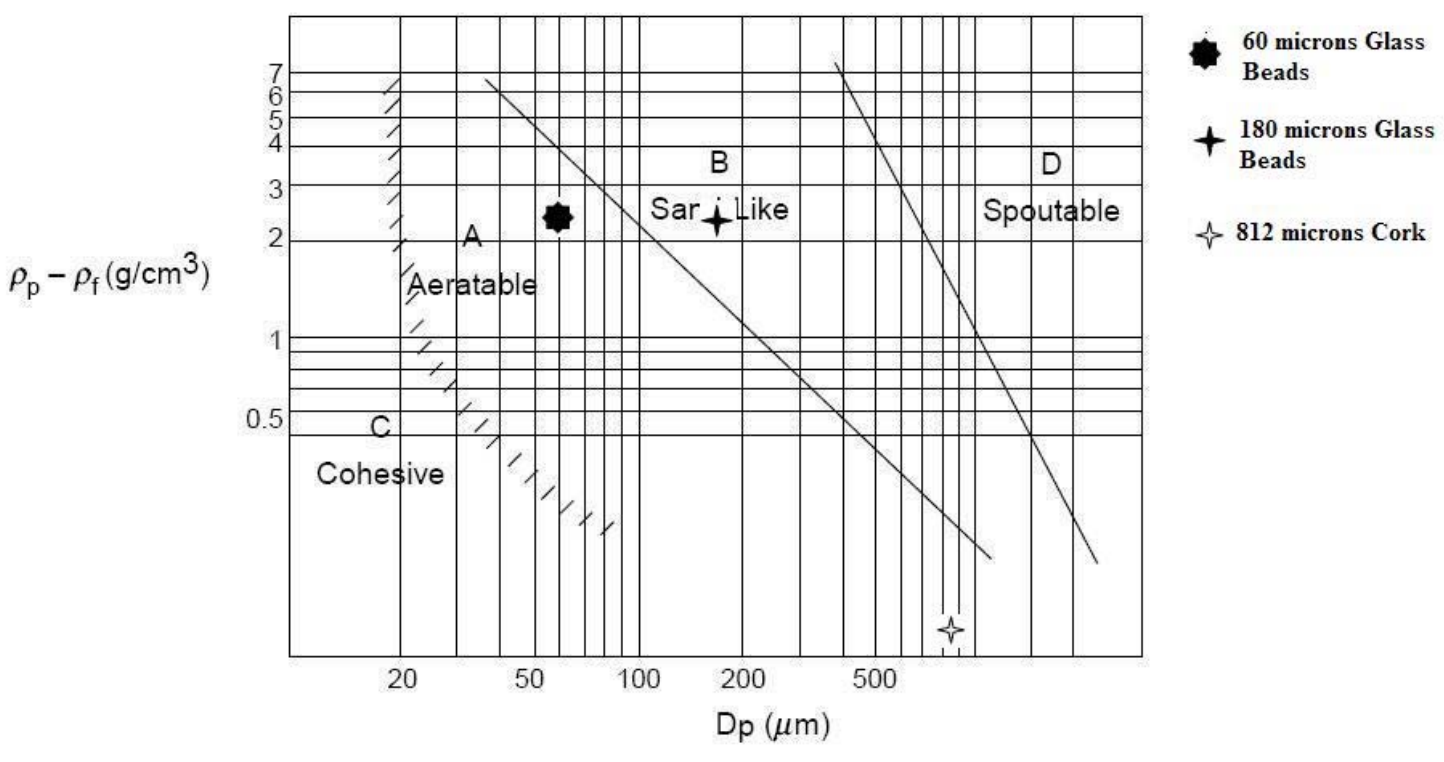

Figure (A1.2): Geldart's classification for the particulate materials used in the thesis.

Geldart's classification for cork and glass beads particulate material used in this thesis at ambient conditions is shown in Fig. (A1.2). The $180 \mu \mathrm{m}$ glass beads with particle density of $2488 \mathrm{Kg} / \mathrm{m}^{3}$ used in the multiple model identification falls under Geldart's Group B category. These particles fluidize well, and bubbles appear as soon as the minimum fluidization velocity is exceeded. On the other hand, cork with particle diameter $812 \mu \mathrm{m}$ and particle density $189 \mathrm{Kg} / \mathrm{m}^{3}$ used in the linear system identification and bed height calculation and glass beads with particle diameter $60 \mu \mathrm{m}$ and particle density $2426 \mathrm{Kg} / \mathrm{m}^{3}$ used 
in the move air flow split estimate and adaptive PID Control, comes under Group A category strictly on the basis of mean size and density as depicted in Fig. (A1.2). However, the operational definition for Group A material is one suggesting they fluidize well, but expand considerably after exceeding the minimum fluidization velocity and before bubbles start appearing, and in which air is trapped once the bed is fluidized resulting in significant time for de-fluidization or de-aeration. Both of these bed materials had relatively narrow size distributions and as a result readily de-fluidize once the aeration is withdrawn. This is more a characteristic of Group B materials. Geldart cautions about the strict application of these guidelines and indicates that materials which are border line may go either way. Both these materials fall in the borderline category, but were thought to behave more like Group B materials, Shadle et. al. [23].

\section{A2 Components of a NETL Cold Flow Circulating Fluidized Bed}

In this thesis, the research unit present at NETL is considered. The circulating fluidized bed under consideration is a "Cold Flow Circulating Fluidized Bed" which means no reaction or combustion is taking place within the unit. The test unit was built at NETL for studying the behavior and operation of circulating fluidized bed systems under cold conditions.

Referring to Fig. (1.1), the various components of the CFCFB that are important in circulating solids under cold conditions around a closed loop through the application of aerations fed across the riser, the loop seal or an L-valve and the standpipe, are described in the following subsections.

\section{A2.1 Riser}

The riser is the most important component in a CFCFB to achieve uniform reaction kinetics, excellent mixing dynamics and stable thermal gradients. Gas-solids flow behaviors in the riser are strongly dependent upon pressure drops of different sections, Kim et. al.[58]. To obtain distribution of solids inventory, not only the mass balance but also the overall pressure balance is needed. As opposed to placing pressure controlling elements in the circulating loop the solids inventories themselves within the riser and standpipe provide pressure balancing mechanism, Lawson et. al.[21].

At high gas velocities in the riser, the limit of solids mass flux in the riser is set by the height of solids in the standpipe. This is because the low height of solids in the standpipe cannot develop adequate head to push the solids into the riser at a rate high enough to equal the maximum solids saturation carrying capacity of the gas flowing through the riser. The solids flow rate in the riser naturally cannot reach its choking value for the particular gas velocity.

When the fluidizing velocity in the riser is low, but the level of solids in the standpipe is high, the limiting mass flux in the riser would depend on the solids inventory of the riser. In this case, the height of solids in the standpipe can push solids into the riser, exceeding its solids flow rate at that gas velocity, which creates a dense-phase at the bottom of the riser, Knowlton [59].

\section{A2.2 Cross Over}

Solids leaving the top of the furnace are fed to the gas-solids separator through a horizontal section known as cross over. On the basis of the study of Patience et. al. [60], the pressure drop across the cross over is defined in terms of solids circulation rate and gas velocity in the horizontal section.

It has been known that the axial solids hold up in the riser is largely affected by operating conditions and the exist geometry, Grace [61], Kunii et. al. [62], Pugsley et. al. [63] and Gupta et. al. [64]. Hence, the effect of the exit configuration on the axial pressure profile should be accounted for in a riser with an abrupt exit during modeling phase. 


\section{A2.3 Gas-Solids Separator}

Gas-solids separation plays an important role in the performance of a CFCFB. A cyclone is a simple device providing a high degree of separation for a minimum pressure drop. The pressure loss across the cyclone is usually assumed to be dependent on the gas velocity, Lei et. al. [65], [61], [62], [63], [64], Rhodes et. al. [66] and Yang [67].

Solids collected in the gas-solids separator drag down some gas as they descend through the standpipe. The amount of gas dragged down could at times be so great that it prevents a dense-phase from forming at the bottom of the standpipe. When this happens, the head that drives the solids through the CFCFB is lost, bringing instability to the entire system. Also Wirth [68] found that if the solids mass flux in the standpipe increased above a certain value, the standpipe connecting the cyclone and loop seal might loose its dense phase seal at the bottom. To avoid this one can reduce the mass flux through the standpipe, decrease the solids circulation rate or increase the diameter of the standpipe.

\section{A2.4 Standpipe}

The standpipe is the vertical pipe connecting the cyclone and the loop seal (or L-valve). In the standpipe the solids are likely to be falling in some mix of either a moving bed, where wall friction and incipient flow dominate, or in a counter-current fashion, fluidized bed, [21]. In either case, the pressure developed from top to bottom will depend on the location and relative velocities $\left(U_{\text {slip }}\right)$ of standpipe aeration gas nozzles. The relative velocities are important rather than the absolute gas velocities is due to the fact that when solids travel down the standpipe with some velocity, they drag some air with them and the absolute value of gas in the standpipe could be either upwards or downwards. What then becomes important is a relative velocity, the vector difference between solids and gas velocities with reference to the standpipe wall.

Solids in the standpipe can move in any of three modes:

A2.4.1 Moving packed bed $\left(U_{\text {slip }}<U_{m f}\right)$

A2.4.2 Fluidized bed $\left(U_{m b}>U_{\text {slip }}>U_{m f}\right)$

A2.4.3 Dilute phase

The dilute phase occurs when the solids concentration is too low compared to the gas flow rate through the standpipe.

\section{A2.5 Solids Recycle Systems}

The recycle system moves the solids from the low-pressure cyclone to the high-pressure bottom of the riser. The amount of solids to be moved is so large that it is nearly impossible to find an inexpensive, motorized mechanical device to transfer it from the standpipe to the riser. A simple device called the loop seal with no moving parts is used to move a large amount of solids from the low-pressure cyclone to the high-pressure end of the riser of a CFCFB. As the loop seal transfers the solids across a pressure barrier which allows the solids to move from the standpipe to the riser but does not allow the gas to move from riser to the standpipe it is also called a non-mechanical valve. Air assists the movement of solids through this valve.

Loop seal is also an important device for CFCFB operation since a short interruption of this device will fill the cyclone with solids and stop the entire cold set up. It consists of a weir and vertical aeration sections and it works best with Group A and B particles. 
The simplest form of non-mechanical valve is a horizontal, L-valve. Some aeration is required along the length of an L-valve to enable solids movement; but the narrow range of control than in loop seal provided by this aeration is of limited value in controlling loop performance. Unlike loop seals, L-valves do not work well with Group A. They work best with Group B and experience difficulties with Group C particles.

\section{A3 Pressure Balance Mechanism in a NETL CFCFB}

To explain the working of the CFCFB loop, we use a simple experiment given in Basu [69]. As shown in Fig. (A3.1), the glass vessel with two columns, (AB and $\mathrm{CD}$ ) is filled with water. Under normal conditions, the water will remain stationary in the column, as static pressures at $\mathrm{B}$ and $\mathrm{C}$ are both equal to the hydrostatic head of water column. Now, when air is injected at the bottom of column CD, streams of bubbles will rise through it and escape out of the top. This will reduce the bulk density of column CD due to the presence of lighter bubbles, which will in turn reduce the static pressure at $\mathrm{C}$ to below that of $\mathrm{B}$. This will cause water to flow from B to $\mathrm{C}$ creating a continuous circulation of water around the vessel. It is analogous to the natural circulation of water and steam in boilers.

This analogy can be applied directly to gas-solid systems in the CFCFB provided the solids in the standpipe behave as a liquid, meaning they deform continuously under the action of shear stress.

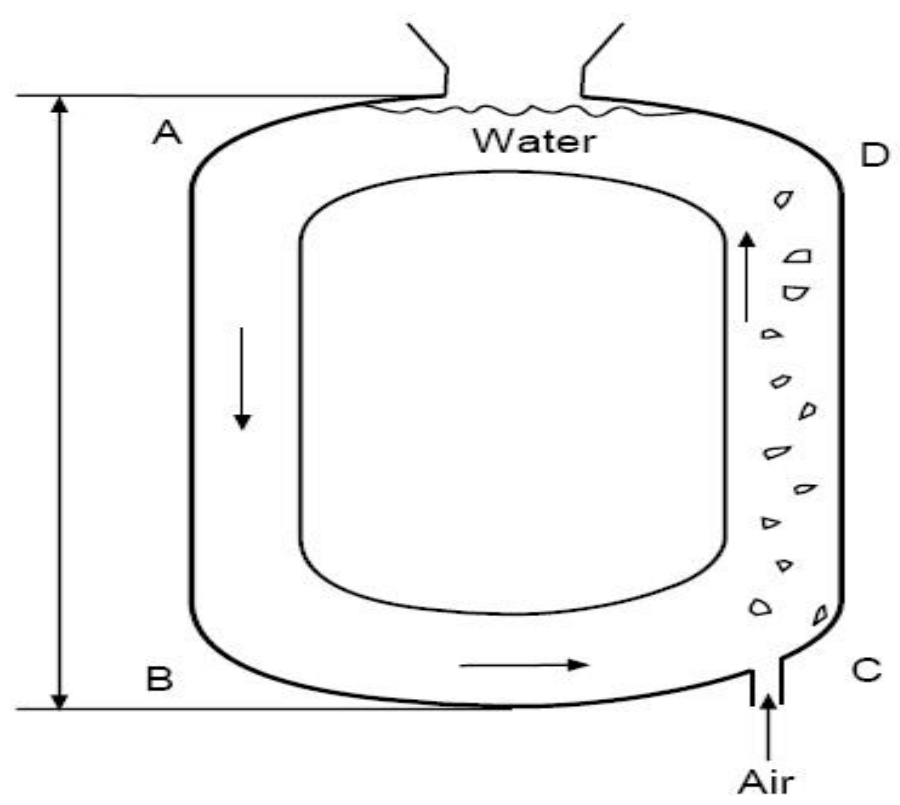

Figure (A3.1): The principle of working a CFCFB can be explained by the example of moving water in a loop by aeration. Ref. [69].

The pressure balance around the cold flow circulating fluidized bed loop can be explained with the help of Fig. (A3.2).

The lower section of the bed is denser and therefore it results in higher pressure drops per unit height of the bed relative to the upper section, which is leaner. For a given amount of bed inventory, the solids are distributed between the bed and the return leg in such a way that the pressure drops across two legs of the loop (Fig. (A3.2)) balance each other out. Thus, we see in Fig. (A3.2) a pressure profile closed around the CFB loop. 


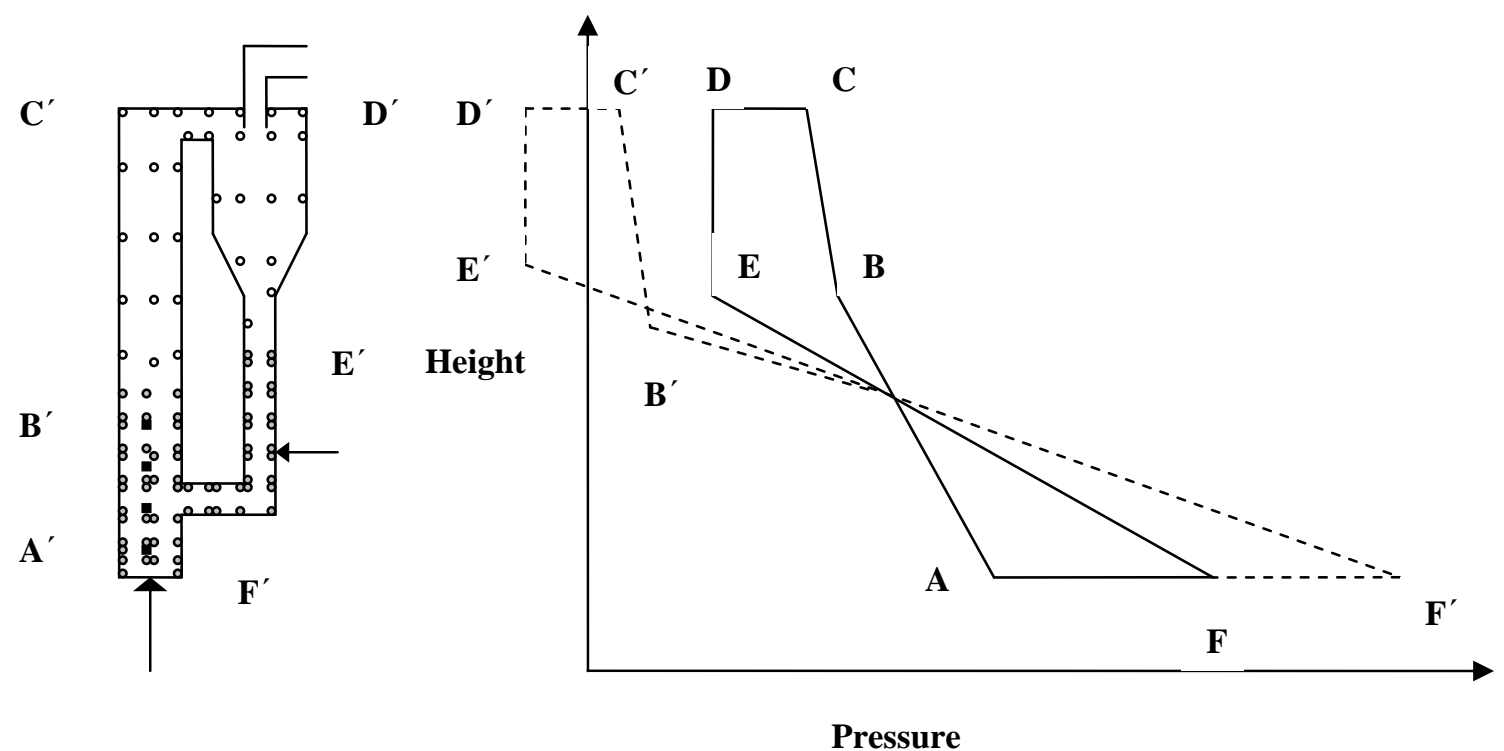

Figure (A3.2): The pressure balance around the CFCFB loop. The dotted lines represent the pressure profile at a higher circulation rate.

The pressure drop across the L-valve controlling the solids flow is proportional to its flow rate. Therefore, when the solids flow-rate is increased by increasing the aeration air, the pressure at point $\mathrm{F}$ (with reference to A) increases, and the pressure drop across the moving packed bed in the return leg increases from $(\mathrm{EF})$ to $\left(\mathrm{E}^{\prime} \mathrm{F}^{\prime}\right)$. The pressure drop across the cyclone also changes from $\mathrm{CD}$ to $\mathrm{C}^{\prime} \mathrm{D}^{\prime}$.

For stable operations the pressure balance around the loop may be written as:

$$
\Delta P_{F-A}+\Delta P_{A-B}+\Delta P_{B-C}+\Delta P_{C-D}=\Delta P_{D-E}+\Delta P_{E-F}
$$

This pressure balance depends on the different operating parameters; hence, the response of the bed to a variation in an operating parameter can be predicted from the pressure balance.

\section{A4 Solids Flow Rate Measurement in a NETL CFCFB}

The particle velocity is a very difficult parameter to determine experimentally. In the CFB risers, pulseinjection techniques have been suggested for its measurements, as well as isolation of a section of the flow system by selenoid valves. The laser Doppler velocimeter system can be employed on dilute systems to measure the solids velocity, Klinzing [70]. Photographic and video techniques can provide information on particle velocity, direction of motion, and acceleration, Yang [71]. However, small fast moving particles are difficult to photograph. Backlighting can be used for contrast in two-dimensional risers, Arena et. al. [72], as well as circular or square columns, Rhodes et. al. [73]. Microcomputer-controlled multicolor stroboscopic photographic (Zheng et. al. [74]), and boroscope optical fiber probes (Takeuchi et. al. [75], Li et. al. [76]) were employed to determine particle velocity, particle acceleration and directions of motion adjacent to the wall, and to study flow structure in the central region of the riser, respectively. 
At the NETL CFCFB, the spiral vane located in the packed bed portion of the standpipe gives continuous measurement of solids velocity, Ludlow et. al. [20]. Solids bulk density then allows calculation of mass circulation rate by incorporating the frequency of its rotation recorded when the solids force it to rotate due to its passage.

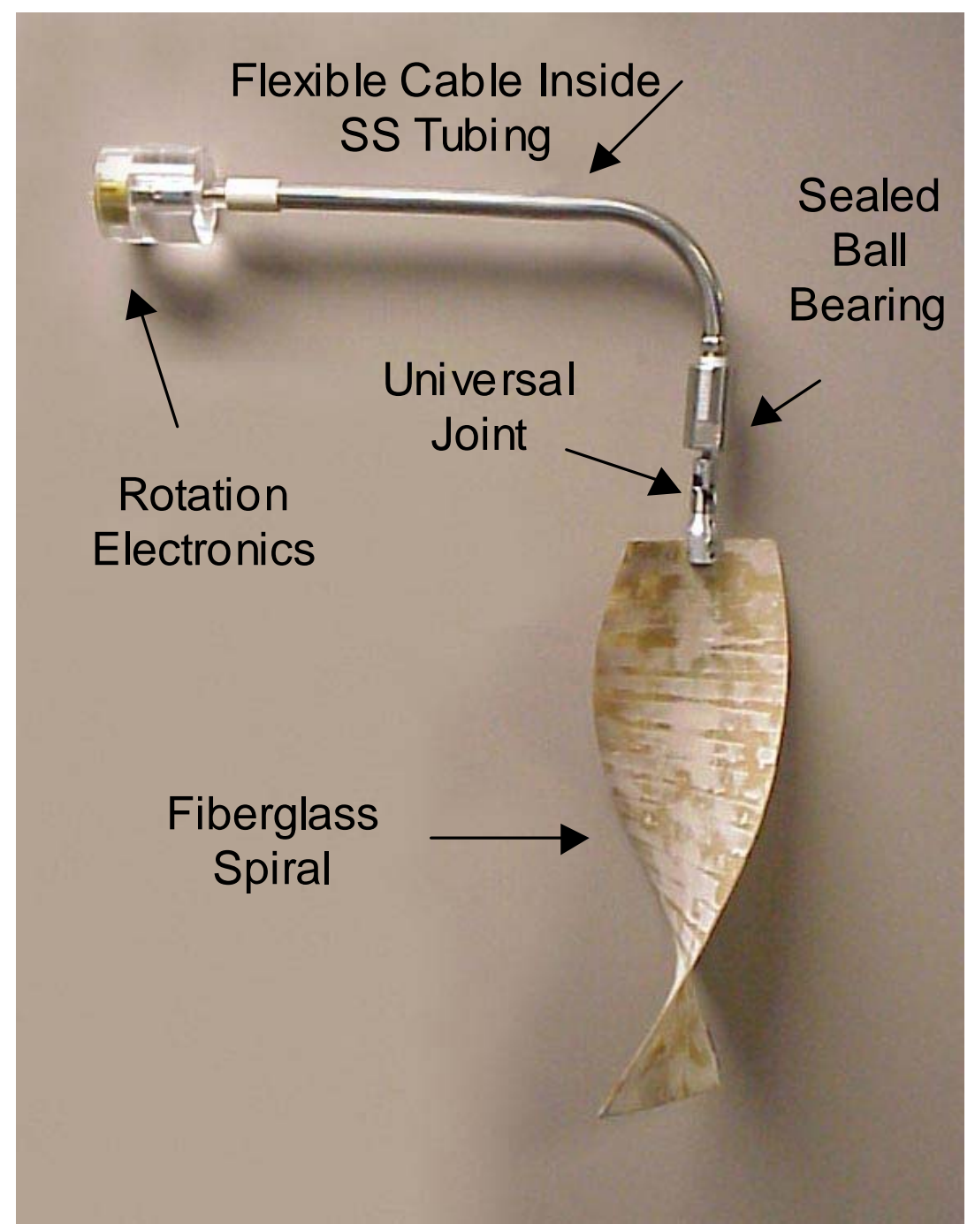

Figure A(4.1): NETL spiral device designed by James Christopher Ludlow, Ludlow et. al. [20].

The helical-shaped fiber glass spiral vane is $30.5 \mathrm{~cm}$ long, $14 \mathrm{~cm}$ wide and has a $180^{\circ}$ twist as pictured in Fig. (A4.1). The most prominent features of this device are that the twist of spiral does not displace bed material as they pass by, that the support structure is so minimal that it can be installed in the unit without any difficulty, and that the acceleration can easily be achieved with spiral during operation because of its small mass. Owing to its design by James Christopher Ludlow in 2001, all the works on modeling, control and others regarding solids flow rate in the CFCFB were possible since then. 


\section{Notations}

\section{Chapter 2}

$\Delta P$

A

$\varepsilon$

$\varepsilon_{\ell}$

$L$

$g$

$\rho_{p}$

$\rho_{g}$

$\Delta P_{T}, L_{s p}$

$\Delta P_{L}, z_{L}$

$\Delta P_{D}, H_{s p}$

$\Delta P, z$

$\Delta P_{h}, h$

\section{Chapter 3}

$y$

$\hat{y}$

$u$

$h$

$p(\varphi)$

$\phi$

$x$

$e, v$

\section{Bed Height Calculation}

Pressure drop across the bed, $\mathrm{Pa}$

Cross-sectional area of the bed, $\mathrm{m}^{2}$

Voidage

Voidage in the lean region of the standpipe

Length of the bed, $\mathrm{m}$

Acceleration due to gravity, $\mathrm{m} / \mathrm{s}^{2}$

Particle density, $\mathrm{Kg} / \mathrm{m}^{3}$

Gas density, $\mathrm{Kg} / \mathrm{m}^{3}$

Total pressure drop across the standpipe, $\mathrm{Pa}$ and length of the standpipe, $\mathrm{m}$

Pressure drop across the lean region of the standpipe, $\mathrm{Pa}$ and length of the lean region, $\mathrm{m}$

Pressure drop across the dense region of the standpipe, $\mathrm{Pa}$ and length of the dense region, $\mathrm{m}$

Pressure drop across the portion of dense region of the standpipe that is always measurably known, $\mathrm{Pa}$ and length of that dense region portion, $\mathrm{m}$

Pressure drop across the portion of dense region of the standpipe that varies according to the bed height level, $\mathrm{Pa}$ and length of that dense region portion, $\mathrm{m}$

\section{Multiple Model Identification of a CFCFB}

Measured SCR normalized to $[0,1]$

Predicted SCR in $[0,1]$ range

Input vector consisting of normalized move air flow, riser aeration and total riser pressure drop

A continuous function with certain smoothness properties

Weighting function for multiple models

Scheduling vector

Fictitious state

Unknown white noise sequence 


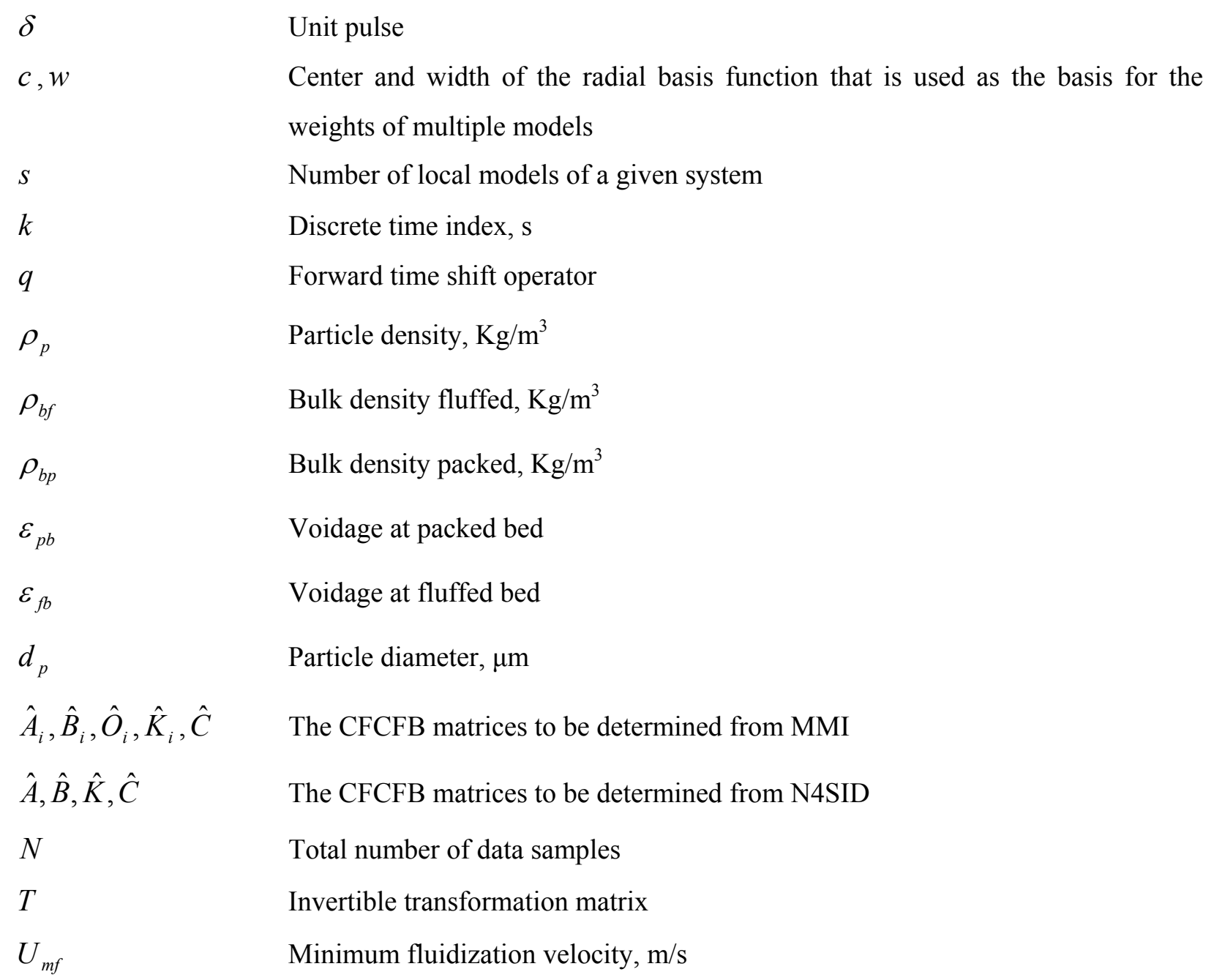

Unit pulse

Center and width of the radial basis function that is used as the basis for the weights of multiple models

Number of local models of a given system

Discrete time index, $\mathrm{s}$

Forward time shift operator

Particle density, $\mathrm{Kg} / \mathrm{m}^{3}$

Bulk density fluffed, $\mathrm{Kg} / \mathrm{m}^{3}$

Bulk density packed, $\mathrm{Kg} / \mathrm{m}^{3}$

Voidage at packed bed

Voidage at fluffed bed

Particle diameter, $\mu \mathrm{m}$

The CFCFB matrices to be determined from MMI

The CFCFB matrices to be determined from N4SID

Total number of data samples

Invertible transformation matrix

Minimum fluidization velocity, $\mathrm{m} / \mathrm{s}$

\section{Chapter 4}

$u_{1}$

$u_{2}$

$u_{3}$

$\left(y_{1}\right), \Delta P_{\text {riser }}$

$\left(y_{2}\right), \Delta P_{\text {crossover }}$

$\left(y_{3}\right), \Delta P_{\text {cyclone }}$

$\left(y_{4}\right), \Delta P_{s \tan d p i p e}$

$\left(y_{5}\right), \Delta P_{\text {valve }}$

$y_{6}$

\section{Linear System Identification of a CFCFB}

Normalized detrended move air flow

Normalized detrended riser aeration

Normalized detrended loop seal fluidization air

(Normalized detrended) total riser pressure drop $(\mathrm{Pa})$

(Normalized detrended) pressure drop across the crossover ( $\mathrm{Pa})$

(Normalized detrended) primary cyclone pressure drop (Pa)

(Normalized detrended) standpipe pressure drop (Pa)

(Normalized detrended) loop seal pressure drop (Pa)

Normalized detrended solids circulation rate 


$\begin{array}{ll}\hat{y}_{i} & \text { Estimated values of dependent variables } \\ e & \text { Innovation terms for } y^{\prime} s \\ \bar{e} & \text { Innovation terms for pressure drops } \\ x & \text { Fictitious state vector } \\ n & \text { Order of the system } \\ m & \text { Number of inputs } \\ \ell & \text { Number of outputs } \\ k & \text { Discrete time index, s } \\ f, g, h & \text { Linear vector function } \\ g_{(d),(s)} & \text { Combined controllability and observability index } \\ A, B, C, K & \text { System matrices } \\ \hat{A}, \hat{B}, \hat{C}, \hat{K} & \text { Estimated system matrices using PEM on input-output data } \\ \hat{A}_{(d),(s)}, \hat{B}_{d}, & \\ \hat{C}_{(d),(s)}, \hat{D}_{(d),(s)}, \hat{K}_{s} & \text { Estimated system matrices for grey box deterministic and stochastic models } \\ W_{c}, W{ }_{o} & \text { Controllability and observability grammians } \\ J & \text { Transformation matrix } \\ M & \text { Inverse of transformation matrix } \\ T & \end{array}$

\section{Chapter $5 \quad$ Air Flow Split and Route Estimation in a CFCFB}

$\begin{array}{ll}\mu & \text { Gas viscosity, Pa.s } \\ \xi & \text { Sphericity of particles } \\ d_{s v} & \text { Surface-volume diameter of particles, } \mathrm{m} \\ \rho_{p} & \text { Solids particle density, } \mathrm{Kg} / \mathrm{m}^{3} \\ t & \text { Continuous time index, } \mathrm{s} \\ k & \text { Discrete time index, } \mathrm{s} \\ \Delta k & \text { Discretization time index for move air flow split, } \mathrm{s} \\ \Delta k_{1} & \text { Discretization time index for move air flow and other standpipe aerations split, } \mathrm{s} \\ a_{t} & \text { Derivative of log of slip velocity between gas and solids, } \mathrm{m} / \mathrm{s}^{2} \\ a(k-1) & \text { Discrete version of } a_{t}\end{array}$




\begin{tabular}{|c|c|}
\hline$a_{1}(k-1)$ & Dynamic parameter of the bottom standpipe after discretization \\
\hline$a_{2}(k-1)$ & Dynamic parameter of the L-valve \\
\hline$b_{l}(t)$ & An approximate of mass flux at the bottom of the standpipe, $\mathrm{Kg} / \mathrm{m}^{2} \mathrm{~s}$ \\
\hline$b_{2}(t)$ & Split parameter for move air flow in the bottom standpipe, $1 / \mathrm{s}$ \\
\hline$b_{t}$ & A dimensionless quantity that is a product of $b_{1}(t)$ and $b_{2}(t)$ \\
\hline$b_{\text {split }}^{1}(k-1)$ & Split parameter for move air flow at the bottom standpipe, $1 / \mathrm{s}$ \\
\hline$b(k-1)$ & Discrete version of $b_{t}$ \\
\hline$b_{L V}, b_{M f}$ & An approximate of mass flux at the bottom of the standpipe and L-valve, $\mathrm{Kg} / \mathrm{m}^{2} \mathrm{~s}$ \\
\hline$b_{\text {split }}^{2}$ & Split parameter for move air flow in the L-valve, $1 / \mathrm{s}$ \\
\hline $\bar{b}_{2}$ & A dimensionless quantity that is a product of $b_{s p l i t}^{2}$ and $\Delta k$ \\
\hline$b_{1 i}$ & Split parameter for move air flow and other aerations, $1 / \mathrm{s}$ \\
\hline$\Delta h_{i}$ & $\begin{array}{l}\text { Height of a section of the standpipe where the path of each standpipe aeration } \\
\text { needs to be determined, } m\end{array}$ \\
\hline$\varepsilon$ & Voidage \\
\hline$\varepsilon_{p b}$ & Gas void fraction in the packed bed \\
\hline$\varepsilon_{m f}$ & Gas void fraction at the minimum fluidization \\
\hline$\varepsilon_{\text {ave }}$ & Average of packed and minimum fluidized gas void fractions \\
\hline$e$ & Measurement noise for pressure drops \\
\hline$w$ & White Gaussian noise for unknown parameters \\
\hline$\lambda$ & Forgetting factor \\
\hline$\tau$ & Time index to designate the old sample with respect to the recent one \\
\hline$f$ & A continuous function with certain smoothness properties \\
\hline$\theta$ & Parameter vector \\
\hline$\hat{\theta}$ & Estimated parameter vector \\
\hline$\varphi$ & An approximate of the gradient with respect to $\theta$ of pressure drop \\
\hline$\Delta P_{\text {bottom }, S P}$ & Pressure drop across the bottom standpipe, Pa \\
\hline$L_{\text {bottom }, S P}$ & Height of the bottom standpipe, $\mathrm{Pa}$ \\
\hline$\Delta P_{L V}$ & Pressure drop across the L-valve, Pa \\
\hline
\end{tabular}




$\begin{array}{ll}U_{s l i p} & \text { Slip velocity between gas and solids, } \mathrm{m} / \mathrm{s} \\ U_{s} & \text { Solids superficial velocity, } \mathrm{m} / \mathrm{s} \\ U_{A e r} & \text { Standpipe aeration vector, } \mathrm{m} / \mathrm{s} \\ U_{A(i)} & \text { Move air flow (other individual standpipe aeration), } \mathrm{m} / \mathrm{s} \\ G_{s} & \text { Solids mass flux, } \mathrm{Kg} / \mathrm{m}^{2} . \mathrm{s} \\ R_{1} & \text { Covariance matrix of white noise } \\ R_{2} & \text { Covariance matrix of white Gaussian noise } \\ K & \text { Kalman gain } \\ Q & \text { Covariance matrix weighted by } \lambda \\ P & \text { Error covariance matrix }\end{array}$

\section{Chapter 6 Adaptive PID Control of a CFCFB}

$\omega_{c}$

$u$

$y$

$r$

$e_{c}$

$a, b$

$\hat{a}, \hat{b}$

$u_{1}$

$u_{2}$

$u_{3}$
Bandwidth optimization parameter of the controller

Forward time shift operator

Input to the plant

Output of the plant

Reference signal according to which output of the plant is to be controlled

Error between reference signal and the output

System scalar quantities

Estimated system scalar quantities using PEM

Move air flow, m/s

Riser aeration, $\mathrm{m} / \mathrm{s}$

L-valve aeration, $\mathrm{m} / \mathrm{s}$

Controlled solids circulation rate, $\mathrm{Kg} / \mathrm{s}$

Parameter vector

Estimated parameter vector

An approximate of the gradient with respect to $\theta$ of $y$

Process noise

Measurement noise 


$\begin{array}{ll}\varepsilon_{p b} & \text { Gas void fraction in the packed bed } \\ \varepsilon_{m f} & \text { Gas void fraction at the minimum fluidization } \\ \varepsilon_{a v e} & \text { Average of packed and minimum fluidized gas void fractions } \\ \rho_{p} & \text { Solids particle density, } \mathrm{Kg} / \mathrm{m}^{3} \\ k & \text { Discrete time index } \\ \lambda & \text { Forgetting factor } \\ \hat{G}_{P} & \text { Estimated plant transfer function } \\ \hat{G}_{C} & \text { Estimated controller transfer function } \\ J_{c} & \text { Cost function between the reference signal and the output of the plant } \\ P & \text { Error covariance matrix } \\ Q_{K} & \text { Covariance matrix weighted by } \lambda \\ K & \text { Kalman gain } \\ U_{s} & \text { Solids superficial velocity, m/s } \\ U_{g} & \text { Gas superficial velocity, m/s } \\ U_{m f} & \text { Minimum fluidization velocity, } \mathrm{m} / \mathrm{s} \\ A_{S P} & \text { Cross-sectional area of the standpipe, } \mathrm{m}^{2} \\ N & \text { Total number of data samples }\end{array}$




\section{References}

[1]. S. Kavidass, G. L. Anderson, and G. S. Norton, Jr., "Why Build a Circulating Fluidized Bed Boiler to Generate Steam and Electric Power", Power Generation Asia 2000, Bangkok, Thailand, Sept. 20-22, 2000.

[2]. L. G. Gilibaro, Fluidization Dynamics, Butterworth-Heinemann, Oxford, 2001.

[3]. D. Wiegan, "Technical and Economical Status of FBC in West Germany", International Conference on Coal Combustion, Copenhagen, 1986.

[4]. B. N. Gaglia, and A. Hal, "Comparison of Bubbling and Circulating Fluidized Bed Industrial Steam Generation", Proc. of $9^{\text {th }}$ International Conference on FBC, Boston, 1987, Vol. 1, pp. $18-25$.

[5]. D. Blauw, and J. O. Donell, "Technology Assessment and Design of Circulating Fluidized Bed Boiler Project for Iowa State University Physical Plant", Proc. of $\mathbf{9}^{\text {th }}$ International Conference on FBC, Boston, 1987, Vol. 2, pp. 1089-1095.

[6]. U. Renz, "State of Art in Fluidized Bed Coal Combustion in Germany", Yugoslav-Germany Colloquium Low-Pollution and Efficient Combustion of Low-Grade Coals, Sarajevo, 1986. Sarajevo, Yugoslavia: Academy of Science of Bosnia and Herzegovina, Department of Technical Science, Special Publications, 1987, pp. 185-214.

[7]. P. Basu, and P. K. Halder, "A New Concept of Operation of a Pulverized Coal Fired Boiler as Circulating Fluidized Bed Firing", Proc. of $9^{\text {th }}$ International Conference on FBC, Boston, 1987, Vol. 2, pp. 1035-1043.

[8]. R. Tollet, E. M. Friedman, D. Parham, and W. J. Larva, "Start-up Activities at the Black Dog AFBC Conversion", Proc. of $\mathbf{9}^{\text {th }}$ International Conference on FBC, Boston, 1987, Vol. 1, pp. 153-160.

[9]. P. Basu, "Design Considerations for Circulating Fluidized Bed Combustors", Journal of the Institute of Energy, 1986, Vol. 441, pp. 175-179.

[10]. L. Reh, "Challenges of Circulating Fluid Bed Reactors in Energy and Raw Materials Industries", Circulating Fluidized Bed Technology-VI, Ed J. Werther, Dechema, e.v, Frankfurt, 1999, pp. $10-11$.

[11]. A. M. Squires, "Fluidized Bed Combustion and Applications", Applied Science Publishers, Ed. J. R. Howard, Barking, 1983, p.278.

[12]. J. Park, Y. Huang, R. Turton, P. Famouri, and E. J. Boyle, “ The Control of Bed Height and Solids Circulation Rate in the Standpipe of a Cold Flow Circulating Fluidized Bed", Powder Technology, 2005, Vol. 150, pp. 176-184.

[13]. Y. Huang, Dynamic Model of Circulating Fluidized Bed, PhD Thesis, West Virginia University, https://eidr.wvu.edu/files/4823/Huang_Yue_dissertation.pdf, 2006. 
[14]. W. N. Sams, “A Model for State and Parameter Estimation in a Circulating Fluidized Bed”, Proc. of the 16th International Fluidized Bed Combustion Conference, Reno Nevada, 2001.

[15]. H. Shim, P. Famouri, W. N. Sams, and E. J. Boyle, "A State Estimation of the Standpipe of a Circulating Fluidized Bed Using an Extended Kalman Filter", Proc. of the $16^{\text {th }}$ International Conference on Fluidized Bed Combustion, May, 2001, pp. 130-140.

[16]. A. A. Jalali, J. Park, P Famouri, E. J. Boyle, and R. Turton, "H $\mathrm{H}_{\infty}$ Based State Estimation on Standpipe of a Cold Flow Circulating Fluidized Bed", Systems, Man and Cybernetics, IEEE International Conference, Oct, 2003, Vol. 3, pp. 2320-2325.

[17]. J. Park, P.Famouri, Y. Huang, R.Turton, and E. J. Boyle, "Bed Height Calculation and Solid Circulation Rate Estimation in Standpipe of CFB", CCCT 2003 Proc., Orlando, FL, USA, July, 2003, pp. 119-123.

[18]. R. Panday, P.Famouri, R.Turton, and E. J. Boyle, "Identification of the Standpipe of a CFCFB with Minimal Number of Pressure Variables”, CCCT 2006 Proc., Orlando, FL, USA, July, 2006.

[19]. A. Davari, P. Koduru, and L. Shadle, "Neural Network Control of a Cold Flow Circulating Fluidized Bed", System Theory, 2003 Proc. of the $35^{\text {th }}$ Southeastern Symposium, March, 2003.

[20]. J. C. Ludlow, L. Lawson, L. J. Shadle, and M. Shyamlal, "Development of a Spiral Device for Measuring the Solids Flow in a Circulating Fluidized Bed", Proc. of the $7^{\text {th }}$ International Conference on Circulating Fluidized Beds (CFB7), Eds. Grace, Zhu, de Lasa, Niagara Falls, Ontario, Canada, May 5-8, 2002, pp. 513-520.

[21]. L. O. Lawson, and L. J. Shadle, "Characterization and Indirect Control of Solids Transfer Loops", Proc. of the $\mathbf{1 5}^{\text {th }}$ International Conference on Fluidized Bed Combustion, FBC 99-0117, 1999.

[22]. L. J. Shadle, E. R. Monazam, and J. S. Mei, “ Circulating Fluid Bed Operating Regime”, International Conference on Circulating Fluid Bed, Niagara Falls, New York, 2002, pp. 255262.

[23]. L. J. Shadle, E. R. Monazam, and J. S. Mei, “ Transient Characterization of Type B Particles in a Circulating Fluidized Bed Riser”, 2007 ECI Conference on The $12^{\text {th }}$ International Conference on Fluidization - New Horizons on Fluidization Engineering, Eds. F. Berruti, X. Bai, and T. Pugsley, 2007, No. 14.

[24]. V. Verdult, L. Ljung, and M. Verhaegen, "Identification of Composite Local Linear State-Space Models using a Projected Gradient Search", International Journal of Control, 2002, Vol. 75(16/17), pp. 1385-1398.

[25]. L. Ljung, System Identification: Theory for the User, New Jersey: Upper Shaddle River, Prentice Hall PTR., 1999.

[26]. P. Van Overschee, and B. De Moor, "N4SID: Subspace Algorithms for the Identification of Combined Deterministic-Stochastic Systems", Automatica, 1994, Vol. 30, pp. 75-93. 
[27]. P. Van Overschee, and B. de Moor, "Closed Loop Subspace System Identification", ESATSISTA/TR1996-521. Katholieke Universiteit Leuven: ftp://ftp.esat.kuleuven.ac.be/pub/sista/vanoverschee/reports/clos.ps.Z

[28]. R. Panday, P. Famouri, B. D. Woerner, R. Turton, L. J. Shadle, J. C. Ludlow, and E. J. Boyle, "Multiple Model Identification of a Cold Flow Circulating Fluidized Bed", $\mathbf{8}^{\text {th }}$ International Circulating Fluidized Bed Technology Conference, 2008, Hamburg, Germany.

[29]. Z. Gao, "Scaling and Parameterization Based Controller Tuning", Proc. of the 2003 American Control Conference, Jun., 2003, pp. 4989-4996.

[30]. R. H. Perry, and D. W. Green (Eds.), Perry's Chemical Engineers Handbook, Mc.Graw- Hill, Seventh Edition, 1997.

[31]. D. Geldart (Ed.), Gas Fluidization Technology, John Wiley \& Sons, 1986.

[32]. T. A. Johansen, R. Shorten, and R. Murray-Smith, "On the Interpretation and Identification of Dynamic Takagi-Sugeno Fuzzy Models", IEEE Transactions on Fuzzy Systems, 2000, Vol. 8(3), pp. 297-313.

[33]. J. Stark, D. S. Broomhead, M. E. Davies, and J. Huke, "Takens Embeddings Theorems for Forced and Stochastic Systems", Nonlinear Analysis, Theory, Methods and Applications, 1997, Vol. 30(8), pp. 5303-5314.

[34]. J. Stark, "Delay Embeddings for Forced Systems part I: Deterministic Forcing", Journal of Nonlinear Science, 1999, Vol. 9, pp. 255-332.

[35]. J. Sjöberg, and L. Ljung, "Overtraining, Regularization and Searching for a Minimum, with Applications to Neural Networks", International Journal of Control, 1995, Vol. 62, pp. 13911407.

[36]. R. Murray-Smith, and T. A. Johansen, Multiple Model Approaches in Modeling and Control, London: Taylor and Francis, 1997b.

[37]. M. J. Rhodes, and P. A. Laussman, "Study of the Pressure Balance around the Loop of a CFB", Can. J. Chem. Eng., 1992, Vol. 70, pp. 625-630, 1992.

[38]. T. Kailath, Linear Systems, Englewood Cliffs, N.J., Prentice-Hall, 1980.

[39]. A. J. Laub, M. T. Heath, C. C. Paige, and R. C. Ward, "Computation of System Balancing Transformations and Other Applications of Simultaneous Diagonalization Algorithms", IEEE Trans. Automatic Control, AC-32, 1987, pp. 115-122.

[40]. B. Moore, "Principal Component Analysis in Linear Systems: Controllability, Observability, and Model Reduction”, IEEE Transactions on Automatic Control, 1981, AC-26, pp. 17-31.

[41]. A. J. Laub, "Computation of Balancing Transformations", Proc. ACC, San Francisco, 1980, Vol.1, paper FA8-E.

[42]. A. W. Bowman, and A. Azzalini, Applied Smoothing Techniques for Data Analysis, Oxford University Press, 1997. 
[43]. R. C. Brown, and E. Brue, "Resolving Dynamical Features of Fluidized Beds from Pressure Fluctuations", Powder Technology, 2001, Vol. 119, pp. 68-80.

[44]. F. Johnsson, R. C. Zijerveld, J. C. Shouten, C. M. van den Bleek, and B. Leckner, "Characterization of Fluidization Regimes by Time-Series Analysis of Pressure Fluctuations", International Journal of Multiphase Flow, 2000, Vol. 26, pp. 663-715.

[45]. R. C. Zijerveld, F. Johnsson, A. Marzocchella, J. C. Shouten and C. M. van den Bleek, "Fluidization Regimes and Transitions from Fixed Bed to Dilute Transport Flow", Powder Technology, 1998, Vol. 95, pp. 185-204.

[46]. C. Alberto, S. Felipe and C. S. Rocha, "Time-Series Analysis of Pressure Fluctuation in GasSolid Fluidized Bed”, Brazilian Journal of Chemical Engineering, Jul.-Sept., 2004, Vol. 21, pp. 497-507.

[47]. S. Ergun, "Fluid Flow through Packed Columns", Chem. Eng. Prog., 1952, Vol.48, pp. 89-94.

[48]. T. M. Knowlton, I. Hirsan, and L. S. Leung, "The Effect of Aeration Tap Location on the Performance of a J-Valve", Fluidization, Eds. Davidson and Keairns, Cambridge University Press, 1978, pp. 128-133.

[49]. W-C. Yang, and T. M. Knowlton, "L-valve Equations", Powder Technology, 1993, Vol. 77, pp. 49-54.

[50]. L. Ljung, and T. Söderströrm, Theory and Practice of Recursive Identification, The MIT Press, Cambridge, Massachusetts, London, England, 1983.

[51]. J. G. Ziegler, and N. B. Nichols, "Optimal Settings for Automatic Controllers", Trans. ASME, 1942, Vol. 64, pp. 759-768.

[52]. K. J. Astrom, and T. Hagglund, The Control Handbook, W.S. Levine, Ed. CRC Press and IEEE Press, 1996, p. 198.

[53]. K. J. Astrom, and B. Wittenmark, Adaptive Control, Addison-Wesley, Reading, Massachusetts, 1989.

[54]. S. Townsend, G. Lightbody, M. D. Brown, and G. W. Irwin, "Nonlinear Dynamics Control Using Local Models", Transactions of the Institute of Measurement and Control, 1998, Vol. 20(1), pp. 47-56.

[55]. L. A. Aguirre, and Á. P. Souza, "An Algorithm for Estimating Fixed Points of Dynamical Systems from Time Series", International Journal of Bifurcation and Chaos, 1998, Vol. 8(11), pp. 2203-2213.

[56]. E. T. van Donkelaar, P. S. C. Heuberger, and P. M. J. Van den Hof, "Identification of a Fluidized Catalytic Cracking Unit: An Orthonormal Basis Function Approach", Proceedings of American Control Conference, 1998, pp. 1914-1917.

[57]. D. Geldart, "The Effect of Particle Size and Size Distribution on the Behavior of Gas-Fluidized Beds”, Powder Technology, 1972, Vol. 6, pp. 201-215. 
[58]. S. W. Kim, S. D. Kim, and D. H. Lee, "Pressure Balance Model for Circulating Fluidized Beds with a Loop-seal”, Ind. Eng. Chem. Res., 2002, Vol. 41, pp. 4949-4956.

[59]. T. M. Knowlton, "Standpipes and Return Systems", Circulating Fluidized Beds, Eds. J. R. Grace, A. A. Avidan, and T. M. Knowlton, Blackie Academic and Professionals, London, 1997, pp. 214-260.

[60]. G. S. Patience, J. Chaouki, and B. P. A. Grandjean, "Solids Flow Metering from Pressure Drop Measurement in Circulating Fluidized Beds”, Powder Technology, 1990, Vol. 61, p. 95.

[61]. J. R. Grace, "Influence of Riser Geometry on Particle and Fluid Dynamics in Circulating Fluidized Bed Risers”, Circulating Fluidized Bed Technology V, Eds. M. Kwauk, J. Li, Science Press, Beijing, 1997, p. 16.

[62]. D. Kunii, and O. Levenspiel, "Effect of Exit Geometry on the Vertical Distribution of Solids in Circulating Fluidized Beds. Part I: Solution of Fundamental Equations; Part II: Analysis of Reported Data and Prediction", Powder Technology, 1995, Vol. 84, p. 83.

[63]. T. Pugsley, D. Lapointe, B. Hirschberg, and J. Werther, "Exit Effects in Circulating Fluidized Bed Risers", Can. J. Chem. Eng., 1997, Vol. 75, p. 1001.

[64]. S. K. Gupta, and F. Berruti, "Evaluation of the Gas-Solid Suspension Density in CFB Risers with Exit Effects", Powder Technology, 2000, Vol. 108, p. 21.

[65]. H. Lei, and M. Horio, "A Comprehensive Pressure Balance Model of Circulating Fluidized Beds”, J. Chem. Eng. Jpn., 1998, Vol. 31, p. 83.

[66]. M. J. Rhodes, and D. Geldart, "A Model for the Circulating Fluidized Bed", Powder Technology, 1987, Vol. 53, p. 155.

[67]. W. C. Yang, "A Model for the Dynamics of a Circulating Fluidized Bed Loop", Circulating Fluidized Bed Technology II, Eds. P. Basu, and J. F. Large, Pregamon Press, Oxford, England, 1988, p. 181.

[68]. K. E. Wirth, "Fluid Mechanics of the Downcomer in Circulating Fluidized Beds", In 8 International Conference on Fluidization, AIChE, Eds. C. Laguerie, and J. F. Large, New York, 1995, pp. 567-576.

[69]. P. Basu, Combustion and Gasification in Fluidized Beds, Taylor and Francis Group, LLC, 2006.

[70]. G. E. Klinzing, Gas-Solid Transport, McGraw-Hill Chemical Engineering Series, USA, 1981.

[71]. W. C. Yang, Ed., Handbook of Fluidization and Fluid-Particle Systems, Marcel-Dekker, NY, USA, 2003.

[72]. U. Arena, A. Cammarota, A. Marzocchella, and L. Massimilla, "Solids Flow Structures in a TwoDimensional Riser of a Circulating Fluidized Bed”, J. Chem. Eng. Jpn., 1989, Vol. 22, pp. 236241. 
[73]. M. J. Rhodes, H. Mineo, and T. Hirama, "Particle Motion at the Wall of the $305 \mathrm{~mm}$ Diameter Riser of a Cold Model Circulating Fluidized Bed", Circulating Fluidized Bed Technology III, Eds. P. Basu, M. Horio, and M. Hasatani, Oxford: Pergamon Press, 1991a, pp. 171-176.

[74]. C. G. Zheng, Y. K. Tung, H. Z. Li, and M. Kwauk, "Characteristics of Fast Fluidized Beds with Internals", Fluidization VII, Eds., O. E. Potter, and D. J. Nicklin, New York: Engineering Foundation, 1992, pp. 275-284.

[75]. H. Takeuchi, and T. Hirama, "Flow Visualization in the Riser of a Circulating Fluidized Bed", Circulating Fluidized Bed Technology III, Eds. P. Basu, M. Horio, and M. Hasatani, Oxford: Pergamon Press, 1991, pp. 177-182.

[76]. H. Li, Y. Xia, Y. Tung, and M. Kwauk, "Micro Visualization of Two Phase Structure in a Fast Fluidized Bed", Circulating Fluidized Bed Technology III, Eds. P. Basu, M. Horio, M. Hasatani, Oxford: Pergamon Press, 1991, pp. 177-182. 